\title{
Technical Design and Optimization Study for the FERMI@Elettra FEL Photoinjector
}

\author{
Steve Lidia (Lawrence Berkeley National Laboratory) \\ Giuseppe Penco, Mauro Trovo' (Sincrotrone Trieste)
}

\section{Introduction}

The FERMI@ @lettra FEL project will provide a novel, x-ray free electron laser user facility at Sincrotrone Trieste based on seeded and cascade FEL techniques. The electron beam source and injector systems play a crucial role in the success of the facility by providing the highest quality electron beams to the linac and FEL undulators. This Technical Note examines the critical technology components that make up the injector system, and demonstrates optimum beam dynamics solutions to achieve the required high quality electron beams.

Section 2 provides an overview of the various systems and subsystems that comprise the photoinjector. The different operating modes of the injector are described as they pertain to the different linac configurations driven by the FEL and experimental design. For each mode, the required electron beam parameters are given.

Sections 3 and 4 describe the critical beamline elements in the injector complex: the photocathode and drive laser, and the RF gun. The required drive laser parameters are given at the end of Section 3. Additional details on the design of the photoinjector drive laser systems are presented in a separate Technical Note.

Design considerations for the RF gun are extensively presented in Section 4. There, we describe the variation of the cavity geometry to optimize the efficiency of the energy transfer to the electron beam. A study of the power coupling into the various cavity modes that interact within the bandwidth of the RF drive pulse is presented, followed by a study of the transient cavity response under several models and, finally, the effects on extracted beam quality.

Section 5 describes the initial design for the low energy, off-axis diagnostic beamline. Beam dynamics simulations using ASTRA, elegant, and MAD are presented.

Section 6 presents the optimization studies for the beam dynamics in the various operating modes. The optimized baseline configurations for the beamline and incident drive laser pulse are presented, supported by simulation results from space-charge tracking codes. Optimization of the beam transport through the downstream linac to the FEL undulator entrance requires significant deviations from the canonical 'flat-top' temporal laser pulse distribution at the photocathode. The physics of nonlinear electron current emission are examined to determine the optimum temporal profile of the drive 
laser in order to produce the required linear current ramp at the injector exit. Parametric sensitivity studies are performed around the baseline configurations, and jitter studies are presented that analyze the stability of the solutions. 


\section{Overview of Electron Source and Injection System}

The front-end injection systems of the FERMI@Elettra linac produce the high brightness electron beams that define the performance of the FEL and quality of the x-rays for the users. The electron source and injection system is specifically designed to minimize technical risk and project cost by utilizing existing accelerator structures, and by leveraging from the technical design efforts of other, contemporary x-ray FEL projects. The major components of the injector are shown in Figure 2.1.
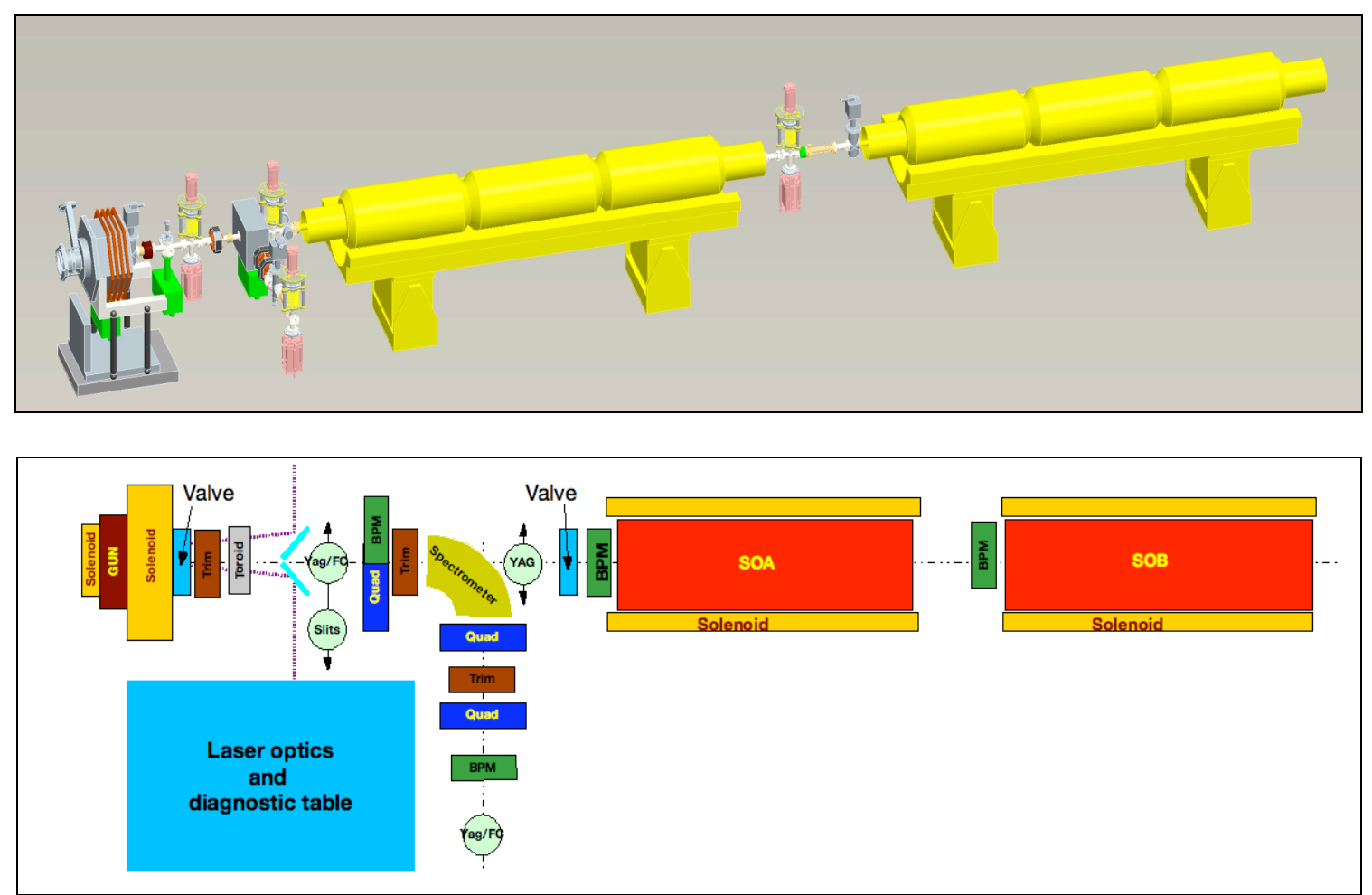

Figure 2.1: The FERMI@Elettra FEL photoinjector.

\subsection{Electron source and RF gun}

The electron source is composed of a metallic photocathode (polycrystalline copper) coupled to an intense, tailored-profile UV laser. Best measured values of the quantum efficiency of copper when illuminated with $263 \mathrm{~nm}$ photons are in the range $10^{-5}-10^{-4}$. Hence, the laser must deliver $\sim 500 \mu \mathrm{J}$ to the cathode in order to produce a bunch with $\sim 1 \mathrm{nC}$ charge.

The photocathode itself provides the conducting back plane to the half-cell of the RF gun. The RF gun operates in the fundamental, $\mathrm{TM}_{010-\pi}$ ('pi') mode of the two-cell cavity, resonant at 2997.9 $\mathrm{MHz}$. The RF gun design is adopted from proven, industrialized technologies, previously optimized for operation at $2856 \mathrm{MHz}$. Small changes in cavity 
geometry are required to change the resonant frequency. However, the detailed design of the cavity instrumentation, RF coupling, vacuum, and thermal handling remain unchanged from the standard design.

The RF gun will provide a peak accelerating gradient of $110 \mathrm{MV} / \mathrm{m}$, and an exit beam energy $\sim 5 \mathrm{MeV}$. This field will be generated by high power, pulsed RF energy originating from a modulator and klystron. Buildup of the cavity field to its nominal value will take approximately $2.8 \mu \mathrm{sec}$ assuming a $10 \mathrm{MW}$ peak input pulse. The initial RF gun will maintain a pulse repetition rate of $20 \mathrm{~Hz}$ or less. Improvements to the cooling circuit will require some additional engineering effort for subsequent RF guns, and will increase the repetition rate to $50 \mathrm{~Hz}$.

External solenoid magnets are integral to the operation of the RF gun. A multiple pancake emittance compensating solenoid provides focusing to transport the beam from the gun exit to the entrance of the booster linac structures. A small bucking coil is used to eliminate any residual magnetic flux threading the photocathode that may result in an increase in the beam emittance.

\subsection{Gun-to-linac (GTL) drift}

The gun-to-linac (GTL) drift section encompasses the beamline from the gun exit to the entrance of the first booster accelerator (see Figure 2.2).

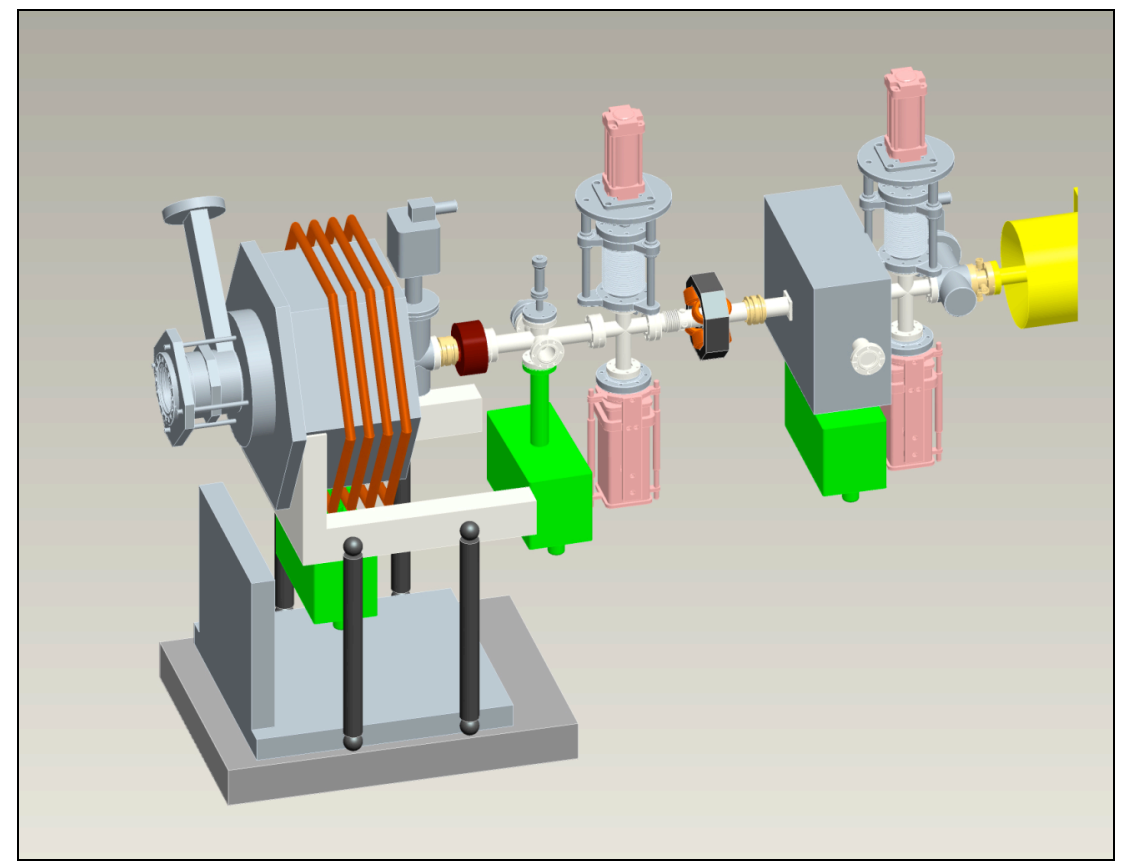

Figure 2.2: Gun-to-linac beamline.

The GTL beamline contains the instrumentation for low energy beam diagnostics, trajectory correction, and vacuum instrumentation for the entire front-end section. Two 
dipole trim magnets allow for corrections to beam offset and angle. Two retractable beam insert vacuum crosses hold beam profile image screens, a charge-measuring Faraday cup, and horizontal and/or vertical slits. An inductive toroid is placed near the gun exit for non-destructive bunch charge measurements. A six-way cross in the beamline allows for on-axis injection of the photocathode drive laser pulse, vacuum pumping and measurement.

Together with the low energy, off-axis diagnostic beamline, the GTL allows for measurement of the transverse and longitudinal beam phase space properties.

\subsection{Low energy diagnostic beamline}

The off-axis, low energy diagnostic beamline concept is shown in Figure 2.3.

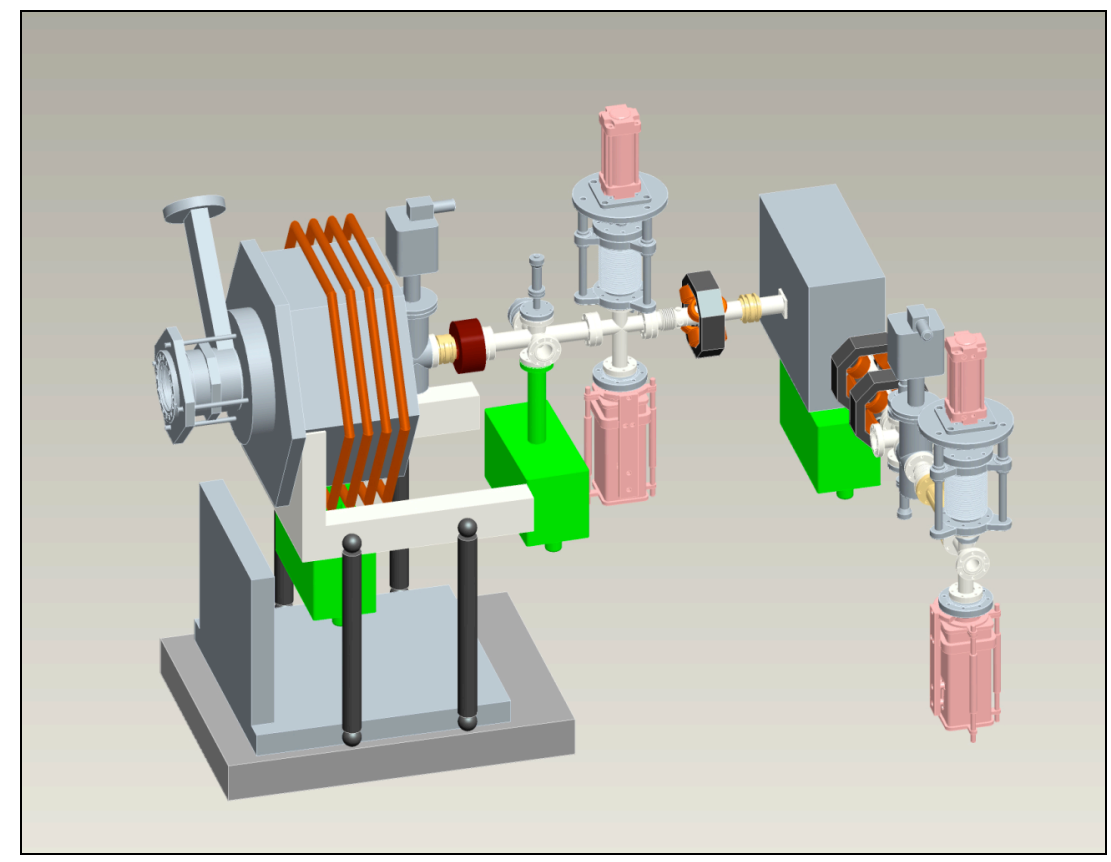

Figure 2.3: Off-axis, diagnostic beamline.

The beamline is comprised of a 90-degree spectrometer magnet, three normal quadrupole magnets, two dipole trim magnets, and a beam profile screen and Faraday cup. The beamline dispersion is tailored by the spectrometer and quadrupole magnets to enhance resolution of energy spread measurements.

\subsection{Booster accelerating modules}

The two booster accelerating structures are shown in Figure 2.1. Each traveling-wave structure is composed of 93 identical cells. Two end cells function to couple RF power into and out of the structure. The active structure length of $\sim 3.3 \mathrm{~m}$ occupies $\sim 3.5 \mathrm{~m}$ of 
beamline. The on-axis, iris coupled cells resonate at $2.998 \mathrm{GHz}$ in the $2 \pi / 3$ mode, and provide peak accelerating gradients of $\sim 19 \mathrm{MV} / \mathrm{m}$, for a total energy gain of $\sim 45 \mathrm{MeV}$ (operationally which includes a 10\% margin). The booster modules include solenoid magnets to provide transverse focusing, to assist with emittance compensation, and to match the optical (lattice) functions at the input to the main linac.

\subsection{Operating modes}

Several configurations of the electron bunch delivered to the undulator chain by the linac accelerator have been considered in the optimization study process, in order to satisfy different requirements coming from the future users' and in the meantime to get flexible the machine design, evaluating different scenarios.

The e-beam dynamics simulation studies have been separated in two parts: the low energy beam tracking (up $100 \mathrm{MeV}$ ) space charge dominated, named injector, and the rest of the linac accelerator. The injector simulations was performed by using two space charge tracking codes: ASTRA [2.1] and GPT [2.2].

As first regime a low charge bunch has been taken in account, consisting in providing at the linac exit a 1kA-bunch with a uniform central core of about 200fs, a projected emittance of $1.5 \mathrm{~mm} \mathrm{mrad}$ and a slice emittance around $1 \mathrm{~mm}$ mrad. This regime has been called "short bunch". The time jitter studies have shown that this regime is not suitable for the FEL seeded scheme with a short laser pulse (about 100fs), which is an interesting option for Fermi. The "short bunch" option is anyway described thanks to the good performance and because it represents an interesting back-up option and/or a start-up operation mode if a long seed laser is used.

In order to accommodate the time jitter coming from the bunch propagation through the linac, without increasing the seed laser length, a longer bunch solution with a higher charge $(\sim 800 \mathrm{pC})$ has been introduced. This regime is called "medium bunch", and it consists in providing a $600 \mathrm{fs}-1 \mathrm{kA}$ bunch at the end of linac. Moreover, a "long bunch" solution has been studied, consisting in more than $1 \mathrm{ps}$ bunch with a moderated peak current around 500 Amps. Following the linac optimization and the compression schemes, the three regimes have been translated in requirements on the bunch at the exit of the photoinjector, summarized in Table 2.1: 
Table 2.1: Main bunch parameters at the exit of the photoinjector in the three options.

\begin{tabular}{|r|c|c|c|}
\hline $\begin{array}{c}\text { Parameters at the exit } \\
\text { of the photoinjector }\end{array}$ & $\begin{array}{c}\text { Short } \\
\text { bunch }\end{array}$ & $\begin{array}{c}\text { Medium } \\
\text { bunch }\end{array}$ & $\begin{array}{c}\text { Long } \\
\text { bunch }\end{array}$ \\
\hline Electron beam energy [MeV] & 95 & 95 & 95 \\
\hline Bunch charge [pC] & 330 & 800 & 1000 \\
\hline Peak current [A] & 60 & 80 & 100 \\
\hline Bunch length (FWHM) [ps] & 5.6 & 9 & 10 \\
\hline Slice emittance [mm-mrad] & $<1$ & $<1$ & $<1$ \\
\hline Projected emittance [mm mrad] & $<1.5$ & $<1.5$ & $<1.5$ \\
\hline Energy spread (uncorrelated) [kev] & $<2$ & $<2$ & $<2$ \\
\hline
\end{tabular}

In the Linac optimization studies, it has been come out that nonlinearity of the waveform of the accelerating field in the linac and nonlinear time-of-flight characteristics of bunch compressors play an important role [2.3]. Starting with a flat top charge distribution, the longitudinal phase space at the end of the linac presents a quadratic chirp, that can dramatically affect the FEL performance [2.4]. It has been found that the phase space portrait at the end of the linac strongly depends upon the peak current distribution at the beginning of the linac, i.e. at the end of the photoinjector. It has been demonstrated [2.5] that in order to compensate the wakefield effects in the linac sections, at the exit of the photoinjector the electron bunch should have a linear ramped peak current distribution instead of a flat top. This requirement is translated in the photoinjector optimization in a big issue due to the strong nonlinearity of the space charge fields at the cathode and in the drift between the gun and the first booster.

In the medium and long bunch regimes ramping current distribution has been evaluated as alternatives to the "classical" flat top bunch, and optimum solutions are presented in the next sections.

\subsection{References}

[2.1] K. Flöttman, https://www.desy.de/ mpyflo/Astra dokumentation.

[2.2] S.B. Van der Geer, et. al., http://www.pulsar.nl/gpt/index.html.

[2.3] A. Zholents, et al., ST/F-TN-06/06, (2006).

[2.4] W. Fawley and G. Penn, ST/F-TN-06/07.

[2.5] A.Zholents, et. al., "Formation of electron bunches for harmonic cascade x-ray free electron lasers”, EPAC'06, Edinburgh, June 2006. 


\section{Photocathode and drive laser systems}

The requirements of future $\mathrm{x}$-ray facilities drive improvements in electron beam quality from reliable, robust sources. The requirements on the electron beam quality necessary for the successful operation of this class of facility are at, or just beyond, the current state of the art. The photoinjector system that produces the high quality electron beam consists of a RF gun, a high peak power UV drive laser system, and a robust metallic photocathode. Techniques for control of the stability and reliability of all three component systems, and on our ability to control the temporal and spatial profiles of the UV laser pulse incident upon the photocathode, are yet to be fully developed.

\subsection{Design considerations for photocathode sources in application to $x$-ray facilities}

The high-brightness electron source must be carefully optimized to minimize and preserve the electron beam emittance, particularly against the deleterious effects of spacecharge and wakefields. Space charge forces have their largest effect at low energies, after the electrons are pulled off the photocathode and before they reach significant kinetic energies (approximately 75-100 MeV). Wakefield effects are more prevalent at higher energies, and require fine tuning of the longitudinal phase space and current profile. To produce a low-emittance beam requires optimization of the illuminating laser pulse temporal and spatial profiles, and energy, accelerating electric field conditions, and magnetic focusing channels.

To support the reliability and availability demanded of a user-facility, the photoinjector is required to be highly stable in operation over hourly, daily, and even weekly timescales. An operational cycle of several months between major maintenance is required. The components of the photoinjector, i.e. the drive laser, photocathode, and RF cavities, are critical elements to achieve these operational reliability specifications.

Applications of high-brightness electron beams in free-electron lasers (FEL's) drive the photoinjector output electron beam specifications that include:

- High charge per bunch, $\mathrm{Q} \sim 1 \mathrm{nC}$

- Small normalized slice emittance, $\varepsilon \sim 1 \mathrm{~mm}-\mathrm{mrad}$

- Small energy spread, $\sigma_{\mathrm{E}} / \mathrm{E}<0.1 \%$

- Control of bunch length from 1 ps to 10's ps

A relatively long electron bunch of $\sim 10 \mathrm{ps}$ duration has potential advantages in reducing the charge density at the photocathode surface or the peak current in the bunch, and thus reducing deleterious space-charge effects. This can lead to reduced emittance electron beams compared to shorter pulse length electron sources that emit few ps duration bunches [3.1]. Manipulation of the longitudinal phase space following acceleration to 
higher energy downstream of the photocathode allows for bunch length compression to generate higher peak current, before utilization of the electron bunches in photonproduction sections. In addition, facilities may demand high pulse repetition rate, synchronization with external clock signals, and high stability:

- Variable repetition rate, $10-50 \mathrm{~Hz}$

- $\quad$ Timing jitter $\sim 100$ - 300 fs

Table 3.1: Photocathode Candidates (courtesy Z.M. Yusof, ANL)

\begin{tabular}{|c|c|c|}
\hline Photocathode & Advantages & Disadvantages \\
\hline $\begin{array}{l}\text { Metallic } \\
\text { ( } \mathrm{Cu}, \mathrm{Mg}, \mathrm{Ag} \text {, etc.) }\end{array}$ & $\begin{array}{ll}\text { - } & \text { Easy to obtain/handle } \\
\text { - } & \text { Widely-used } \\
\text { - } & \text { Rugged, and does not } \\
& \text { require UHV } \\
\text { - } & \text { QE constant for long } \\
& \text { time } \\
\text { - } & \text { Fast response time } \\
\text { - } & \text { Allows for pulse shaping }\end{array}$ & $\begin{array}{ll}\text { - } & \text { Low QE }(<0.01 \%) \\
\text { - } & \text { No systematic study of } \\
\text { effective cleaning \& } \\
\text { rejuvenating method, } \\
\text { especially in-situ at } \\
\text { photoinjector } \\
\text { - } \\
\text { Not indicated for high } \\
\text { average power applications } \\
\text { Beam tracks temporal } \\
\text { fluctuations in drive laser }\end{array}$ \\
\hline $\begin{array}{l}\text { PEA Semiconductor } \\
\left(\mathrm{Cs}_{2} \mathrm{Te}, \mathrm{K}_{2} \mathrm{Te}, \mathrm{GaN} \text {, }\right. \\
\text { etc. })\end{array}$ & $\begin{array}{ll} & \text { High QE }(5-30 \%) \\
\text { - } & \text { Photoelectrons have } \\
& \text { lower energy spread (in } \\
& \text { principle) than metallic } \\
\text { - } & \text { Low dark currents } \\
\text { - } & \text { Allows pulse shaping for } \\
& \text { long pulses (few 10's ps) }\end{array}$ & $\begin{array}{ll} & \text { Requires UHV } \\
\text { - } & \text { Surface deteriorates with } \\
\mathrm{O}_{2} \\
\text { - } \\
\text { Longer response time than } \\
\text { metallic }(\sim \text { ps }) \\
\text { - Initial QE has short } \\
\text { lifetime }\end{array}$ \\
\hline $\begin{array}{l}\text { NEA Semiconductor } \\
\text { (GaAs family, GaP, } \\
\text { etc.) }\end{array}$ & $\begin{array}{ll}\text { - } & \text { High QE (10-60\%) } \\
\text { - } & \text { Possible source of } \\
\text { polarized electrons } \\
\text { (GaAs) } \\
\text { - } \quad \text { Slow emission time } \\
\text { damps laser fluctuations }\end{array}$ & $\begin{array}{ll}\text { - } & \text { Requires UHV } \\
\text { - } & \text { Long response time (10's } \\
& \text { ps) } \\
\text { - } & \text { Difficult for RF gun use }\end{array}$ \\
\hline Dispenser Cathodes & $\begin{array}{ll}\text { - } & \text { Low work function / } \\
\text { High QE } \\
\text { - } \quad \text { Self-repairing \& Robust } \\
\text { - } \quad \text { Mature technology base }\end{array}$ & - Recent innovation \\
\hline
\end{tabular}

\subsection{High quantum efficiency photocathodes}

The quantum efficiency of the photoemissive cathode is the ratio of the number of emitted electrons to the number of incident photons. For various photocathode materials the Q.E. varies from $<10^{-4}$ for copper to $\sim 0.1$ for $\mathrm{GaAs}(\mathrm{Cs}, \mathrm{O})$. To produce an electron beam with charge $Q_{\text {bunch }}$ requires a laser pulse capable of delivering energy 


$$
W_{\text {laser }}=\frac{Q_{\text {bunch }}}{Q . E .} \frac{h c / e}{\lambda_{\text {laser }}},
$$

where $h c / e$ is $1.2398 \mu \mathrm{J}-\mathrm{nm} / \mathrm{nC}$. For example, with a laser wavelength of $263 \mathrm{~nm}$ (or a photon energy of $4.7 \mathrm{eV}$ ) to match the work function of $\mathrm{Cu}$, this energy is approximately $0.5 \mathrm{~mJ}$ per pulse to produce a $1 \mathrm{nC}$ bunch with a photocathode Q.E. of $10^{-5}$. Over time, the Q.E. of the photocathode may droop, requiring larger laser pulse energies to be delivered to the photocathode. For these reasons, the laser system may be specified to deliver $\sim 0.50-1.0 \mathrm{~mJ}$ (UV) per pulse to the photocathode. Laser systems can routinely produce this energy, although losses in transmission from the laser system to the photocathode may be significant.

Properties of different types of photocathodes are summarized in Table 3.1. The use of metallic copper photocathodes is desirable for the proposed initial phases of the FERMI FEL as it mitigates unnecessary risk by ensuring peak reliability and performance in the electron beam source. The metallic cathodes have the required ruggedness to maintain their intrinsic quantum efficiency for long periods, relegating the task of cathode maintenance and replacement to infrequently occurring operations. The fast temporal response allows for various temporal pulse shapes to be generated with the drive laser system and mapped onto the emitted electron beam. Copper cathodes do, however, require UV illumination for efficient photoemission that necessitates higher peak power IR lasers and stages of harmonic generation.

Photocathode performance is dictated and characterized by a relatively small number of parameters to achieve useful emission, namely, bulk temperature, field strength at the cathode surface, work function at the emission site and/or the nature of coatings or contaminants, emission temporal response, and drive laser illumination particulars such as intensity, duration, shape, and pulse repetition frequency (duty factor). Fields on the cathode are generally 10 to $100 \mathrm{MV} / \mathrm{m}$, and generally the cathode is desired to produce $0.1-1 \mathrm{nC}$ in FWHM pulse durations of $10 \mathrm{ps}$ in a disk approximately $4 \mathrm{~mm}^{2}$ in area. Note that $1 \mathrm{nC} / 10 \mathrm{ps}$ corresponds to 100-A peak current, and the field produced by a sheet with a charge density of $\left(1 \mathrm{nC} / 4 \mathrm{~mm}^{2}\right)$ is $\mathrm{Q} /\left(2 \varepsilon_{0} \mathrm{~A}\right) \sim 14 \mathrm{MV} / \mathrm{m}$, so space-charge effects within the disk can be disruptive. These disruptive fields can be diminished by use of pulse lengths of tens of picoseconds or longer.

The generation of the lowest emittance beam from any electron sources requires careful matching of the drive laser parameters to the photocathode. Modern photoinjector design can reduce the emittance growth in the low-energy, space-charge-dominated regime so that the final beam emittance is dominated by the thermal distribution of the electrons at the cathode surface. Recent studies [3.2] have elucidated the relationship between thermal emittance and quantum efficiency in metallic cathodes, which are mediated by strong, time-dependent Schottky effects at the cathode surface. The relationship is shown in Figure 1. Removing excess kinetic energy from photoexcited electrons in the copper photocathode reduces thermal emittance, but at the cost of quantum efficiency. 

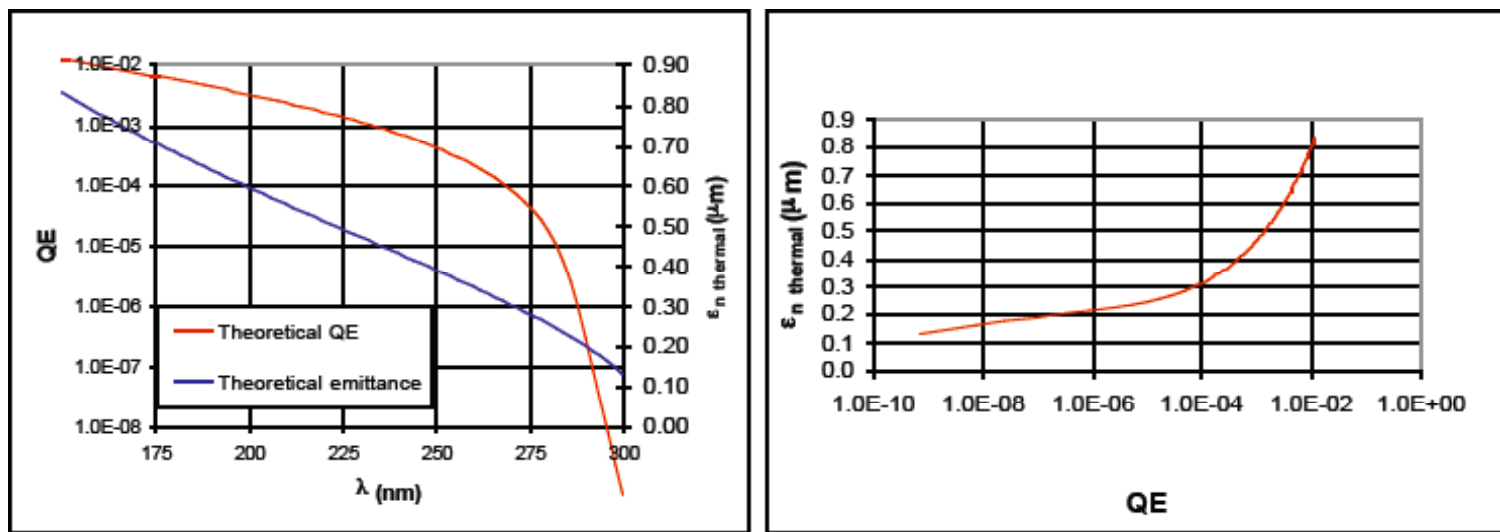

Figure 3.1: Theoretical emittance and QE from $\mathrm{Cu}$ [3.2].

\subsection{Required specifications for the photoinjector drive laser}

Laser systems have already been developed to address some of the needs for photocathode systems described above, but they currently exhibit a highly nonuniform temporal profile, low stability, low repetition rate, or short pulse length [3.3, 3.4]. A goal of this proposal is to demonstrate a laser system that combines the advantages of high stability, precise control over the instantaneous intensity across the photocathode, and over the (potentially relatively long) pulse length.

Given the measured quantum efficiency for typical photocathode materials considered here, and the required typical electron parameters for low-emittance, high-charge bunches, the intensity and time specifications for the UV illumination pulse can be derived. Table 3 lists the output UV pulse temporal specifications. Specifications for the beam transverse profile parameters are listed in Table 3.2.

Table 3.2: Temporal specifications for the UV laser pulse.

\begin{tabular}{|r|c|}
\hline Wavelength & $<270 \mathrm{~nm}$ \\
\hline Required pulse energy at the cathode (Cu) & $0.5-1 \mathrm{~mJ}$ \\
\hline Pulse duration range (FWHM) & $1-20 \mathrm{ps}$ \\
\hline Rise/fall time range (10-90\%) & $0.5-5 \mathrm{ps}$ \\
\hline Instantaneous power flatness (RMS) & $<1 \%$ \\
\hline Synchronization (RMS) & $<300 \mathrm{fs}$ \\
\hline Pulse repetition rate range & $10 \mathrm{~Hz}-50 \mathrm{~Hz}$ \\
\hline
\end{tabular}

Table 3.3: Spatial specifications for the UV laser spot at the photocathode.

\begin{tabular}{|r|c|}
\hline Beam diameter range (FWHM) & $2-4 \mathrm{~mm}$ \\
\hline Edge ramp width (10-90 \%) & $0.5 \mathrm{~mm}$ \\
\hline Intensity flatness (RMS) & $<1 \%$ \\
\hline
\end{tabular}


Beam shaping techniques have employed refractive, reflective, diffractive, and absorptive optics to transform Gaussian-like spatial profiles into uniform, 'flat-top' distributions [3.5]. Individual beam profiling elements have been employed in the past, producing nearly flat-top distributions with $\sim 10 \%$ RMS intensity variations [3.1, 3.6]. A system comprised of multiple beam shapers/correctors may provide a degree of control over the wavefront amplitude and phase to generate the tailored spatial profiles with low $(<1 \%$ RMS) intensity variations required at the photocathode plane.

Previous efforts to produce laser pulses with a controlled temporal profile (not simply Gaussian-like) have had varying degrees of success [3.7, 3.8, 3.9]. One approach is to add a series of short pulses in a pulse-stacker. This method produces noisy interference in the areas where the pulses overlap, creating perturbations in the emitted electron bunch current from a photocathode. Also, energy variations in the initial pulse are nonlinearly amplified in the frequency converter, resulting in uncontrolled variations in pulse shape as well as overall energy.

Shaping of the temporal and spatial laser pulse profile may be performed before after harmonic generation from IR to UV. In previous sections we have discussed the requirements for temporal shaping of the laser pulse profile, for example to obtain a flattop distribution or a ramped distribution of charge versus time. This type of shaping may be generated in several ways. Acousto-optic modulation and spatial light modulation in the Fourier plane are recent examples of temporal pulse shaping for photoinjector drive lasers.

Aspheric optical pulse flatteners [3.10] have recently seen implementation in photoinjector laser systems to efficiently shape the transverse wavefront such that a more-or-less uniform spatial profile is generated at the cathode plane. Results from early tests at SLAC's GTF are shown in Figure 3.2. Additional spatial light modulators may be employed to obtain fine control over the 2D laser pulse intensity profile. Small variations in the local cathode quantum efficiency may appear as 'bright' or 'dark' areas, as shown in Figure 3.3. Fine tuning the 2D spatial profile can then enhance the overall uniformity of charge emission. 


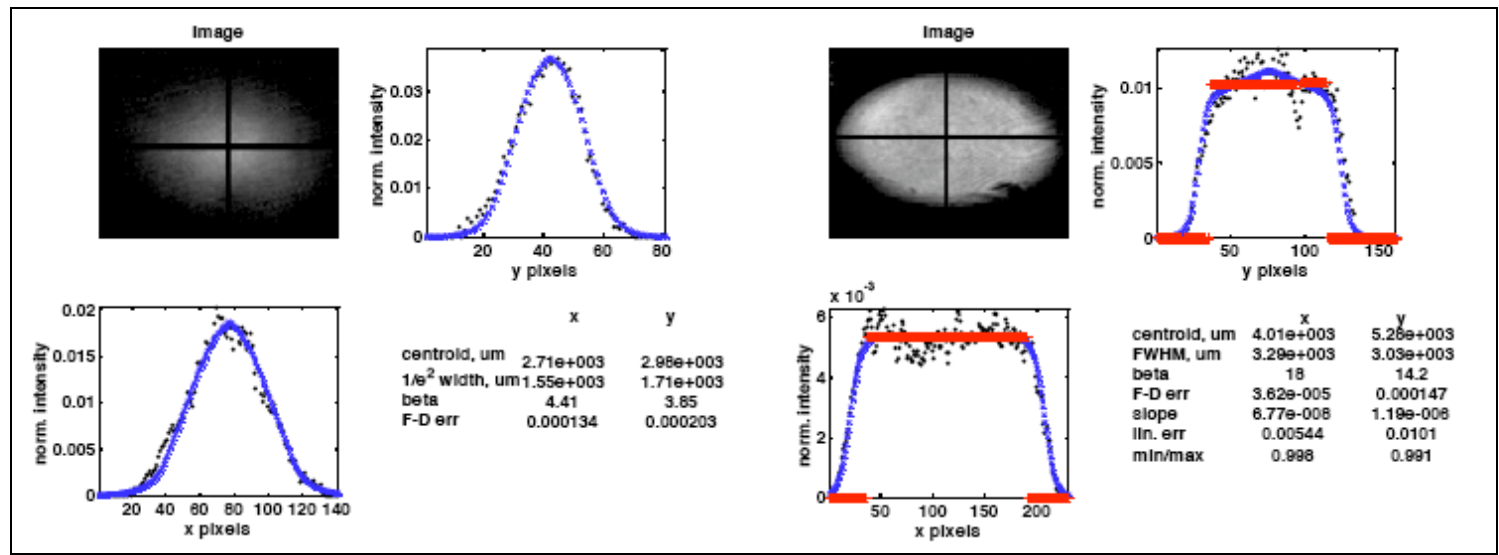

Figure 3.2: Optical pulse flattening in the SLAC GTF photoinjector laser system.

[Courtesy, J. Schmerge.]

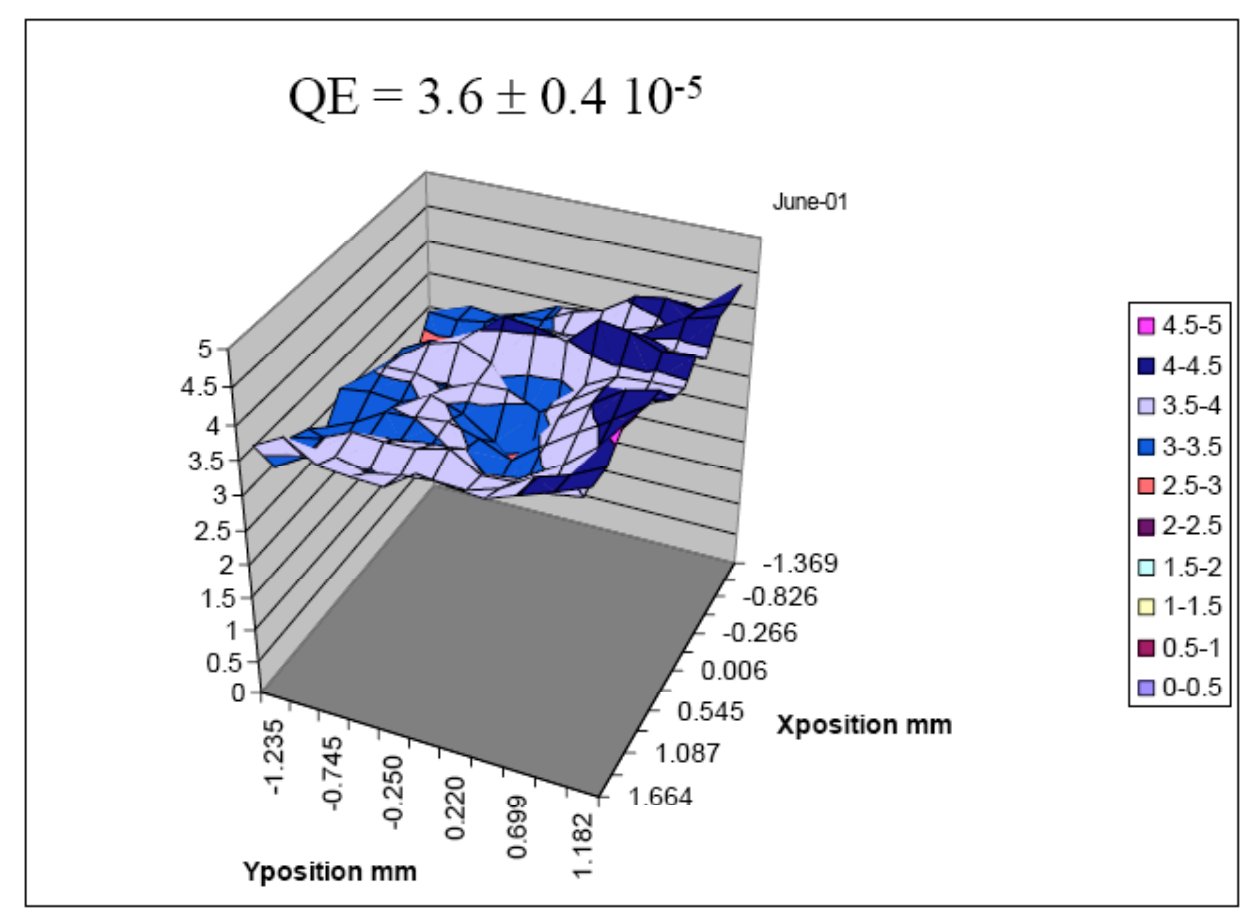

Figure 3.3: Local quantum efficiency variations of copper cathode. [Courtesy, J.Schmerge.]

\subsection{References}

[3.1] H. Tomizawa, et. al., "Reduction of electron-beam emittance with shaping both spatial and temporal profiles of uv-laser light source for photo-cathode RF gun", Proceedings of EPAC 2002, Paris, France.

[3.2] J. Schmerge, et. al., Proceedings of the Physics and Applications of High Brightness Electron Beams, Erice, 2005.

[3.3] A.R. Fry, et. al., Nucl. Instrum. Methods Phys. Res. A 430 (1999), 180.

[3.4] X.J. Wang, et. al., Nucl. Instrum. Methods Phys. Res. A 375 (1996), 82. 
[3.5] F.M. Dickey and S.C. Holswade, eds. Laser Beam Shaping: Theory and Techniques, Marcel Dekker, Inc. (New York, 2000).

[3.6] V. Bagnoud and J.D. Zuegel, Optics Lett. 29 (2004), 295.

[3.7] M. Nisoli, et. al., "Amplitude and phase spectral shaping of fs pulses for lowemittance electron-beam generation", in Proceedings of CLEO 2003, paper CThM21. [3.8] M.J. Fitch, Electro-optic sampling of transient electric fields from charged particle beams, Ph.D. Thesis (U. Rochester, 2000).

[3.9] S. Cialdi, I. Boscolo, and A. Flacco, J. Opt. Soc. Am. B 21 (2004), 1693.

[3.10] J. A. Hoffnagle and C. M. Jefferson, "Design and performance of a refractive optical system that converts a Gaussian to a flat-top beam," Appl. Opt. 39, 5488-5499 (2000). 


\section{RF Gun Design and Optimization}

The high brightness requirement of the electron beam produced by the photoinjector for the FERMI FEL project forces us to examine all possible effects within the RF gun that may result in beam quality deterioration. An issue identified in the current generation of RF guns is the presence of the lower frequency coupled-cavity mode, the 'zero' mode in a two-cavity structure, that may exhibit a finite overlap in its frequency response with that of the drive pulse from the klystron. Significant field oscillation amplitude may be established that can interfere with the detailed beam dynamics of the emitted electrons with the primary accelerating cavity mode (the 'pi' mode), resulting in beam quality deterioration.

We begin with a study of the cavity modes using frequency-domain and time-domain electromagnetic models. The fundamental strategy and purpose of this study is to increase the resonant frequency separation between the two modes in order to decrease the coupling of the zero mode to the klystron drive pulse. This is one technique for eliminating the effect of the zero mode upon the beam dynamics. Other, more active techniques may also be considered.

A variant geometry to the standard pill-box cavity has been investigated. A reentrant type cathode cell allows for tailoring of the transit-time factor while maintaining an optimized shunt impedance $([\mathrm{R} / \mathrm{Q}])$. The two-cell cavity designs presented incorporate varying pi/zero mode frequency separations, while keeping constant the pi-mode frequency and field balance between cells. For the largest foreseeable mode frequency separation, the cavity drive efficiency decreases by $\sim 10 \%$.

\subsection{RF cavity design and optimization}

\section{PI mode optimization studies}

Some RF cavity geometries, proposed for the FERMI photo-gun, are reviewed in this document. The pi-modes are resonant at the European frequency of $2997.9 \mathrm{MHz}$, but they are different in cell lengths and pi/zero mode separation. We calculate for all of them some parameters that are standard Figures of merit for RF cavities.

\section{Cavity characterization}

The RF cavity characterization is a quite standard procedure and the main quantities that depend from the cavity geometry are the resonant frequencies, the field distributions in the volume and the quality factor. Then there are other quantities also describing the cavity proprieties that taken in to account a charge particle which is accelerated along the axis by the cavity fields, as transient time factor, shunt impedance and R/Q. The peculiarity of the photoinjector cavities is that the electrons extracted from the cathode have very low energy and it is incorrect assume $\beta=1$ in the whole particle trajectory. For 
the calculation of the transient time factor and the other parameters depending from the accelerated particle should be taken in to account also the launching phase, i.e. the field phase when the electron is emitted from the cathode.

We report in the following our estimations of some parameters of different RF cavities proposed so far for the FERMI photo-gun. We used SUPERFISH code to calculate the resonant modes, field distribution and the quality factor $(\mathrm{Q})$ and one of our codes, written in Mathcad, for the estimation of the electron energy gain, the transit time and the R/Q. The code loads the electrical field distribution on axis produced by SUPERFISH and calculates the particle trajectory through the cavity. The output parameters are calculated as function of the launching phase.

We start this review with the result of the first frequency scaling performed [4.1] at an earlier stage of the project. The frequency scaling was performed by reducing the $\mathrm{BNL} / \mathrm{SLAC} / \mathrm{UCLA} 1.6 \mathrm{Gun}$ geometry by a factor $\mathrm{K}=f_{B N L} / f_{F E R M I} \approx 0.96$. This scaling effectively raises the resonant frequency from $2856 \mathrm{MHz}$ to $2997.9 \mathrm{MHz}$, but disregards other frequency scaling laws involved in normal conducting RF cavity optimization [4.2].

We have studied several variant cavity geometries with the fundamental, pi-mode frequency resonant at 2997.9 MHz. The cavity geometries and field patterns are shown in Figures 4.1-4.5 below. Case 1 presents the naively scaled geometry. Case 2 shows a more complicated design that leaves the half-cell length constant at the $2856 \mathrm{MHz}$ design value (to avoid excessive redesign of the extensive half-cell instrumentation and port layout), but then varies the half-cell radius, the full-cell radius and length, and the inter-cell iris radius and length. Case 3 takes the basic design from Case 2, but then adds a small (1-2 $\mathrm{mm}$ ) raised mesa at the cathode plane to decrease beam RF transit angle in the half cell, thus permitting a more advantageous launch phase of the beam. Case 4 examines a similar geometry with a slightly small mesa height. Case 5 then alters the inter-cell iris geometry by introducing an elliptical cross-section that can significantly reduce the peak surface fields in the cavity, lowering the breakdown probability and raising the achievable accelerating gradient.

Figure 4.1: Case 1

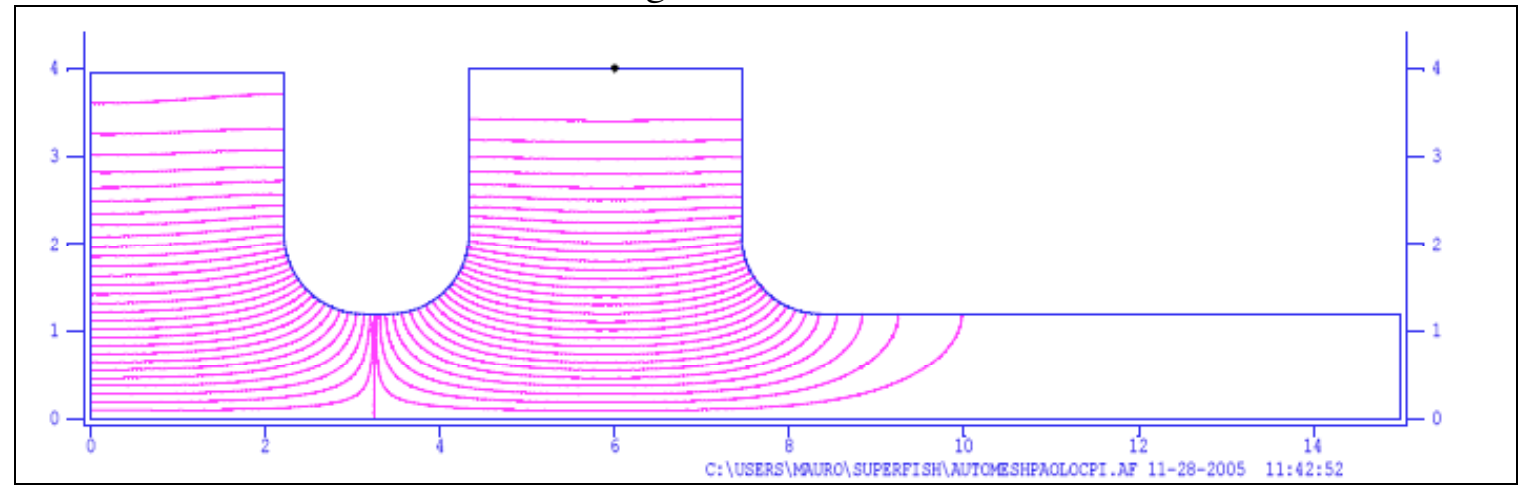


Figure 4.2: Case 2

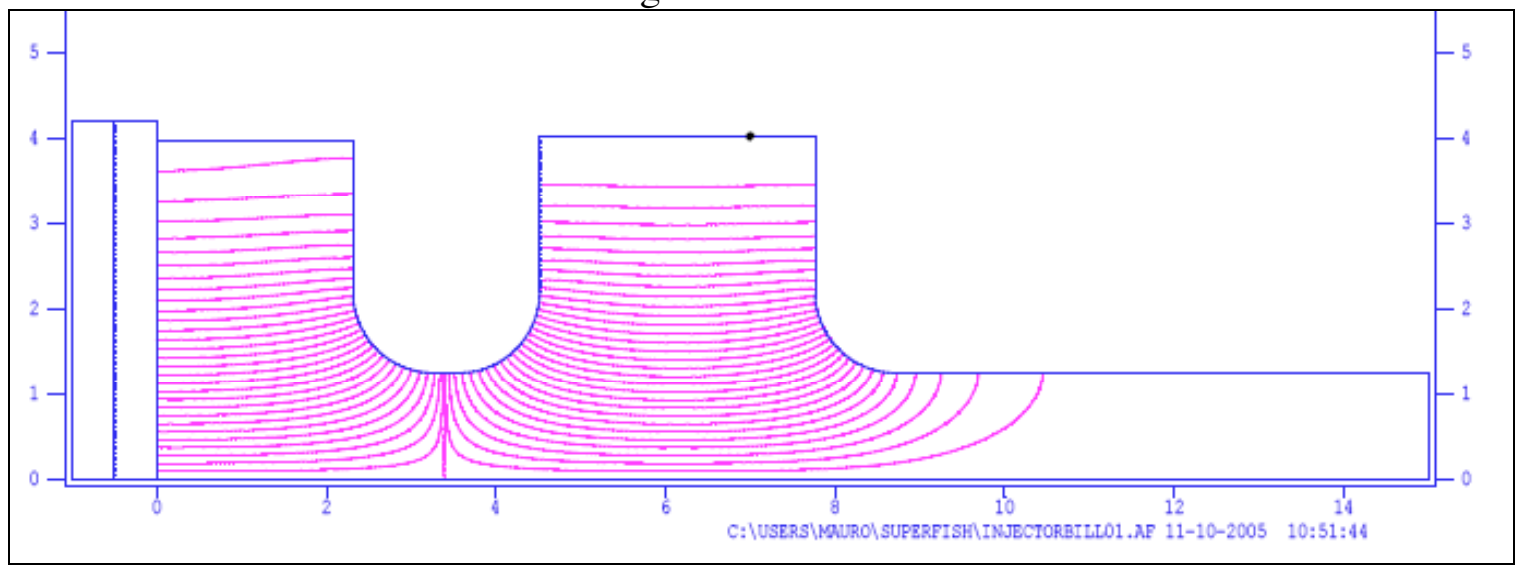

Figure 4.3: Case 3

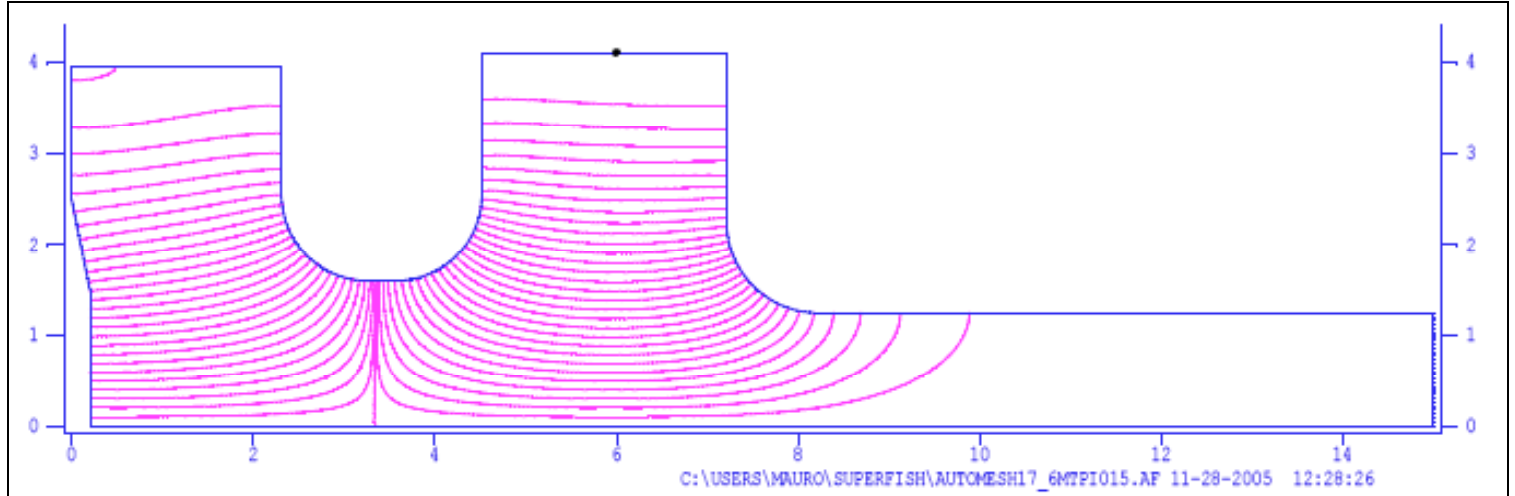

Figure 4.4: Case 4

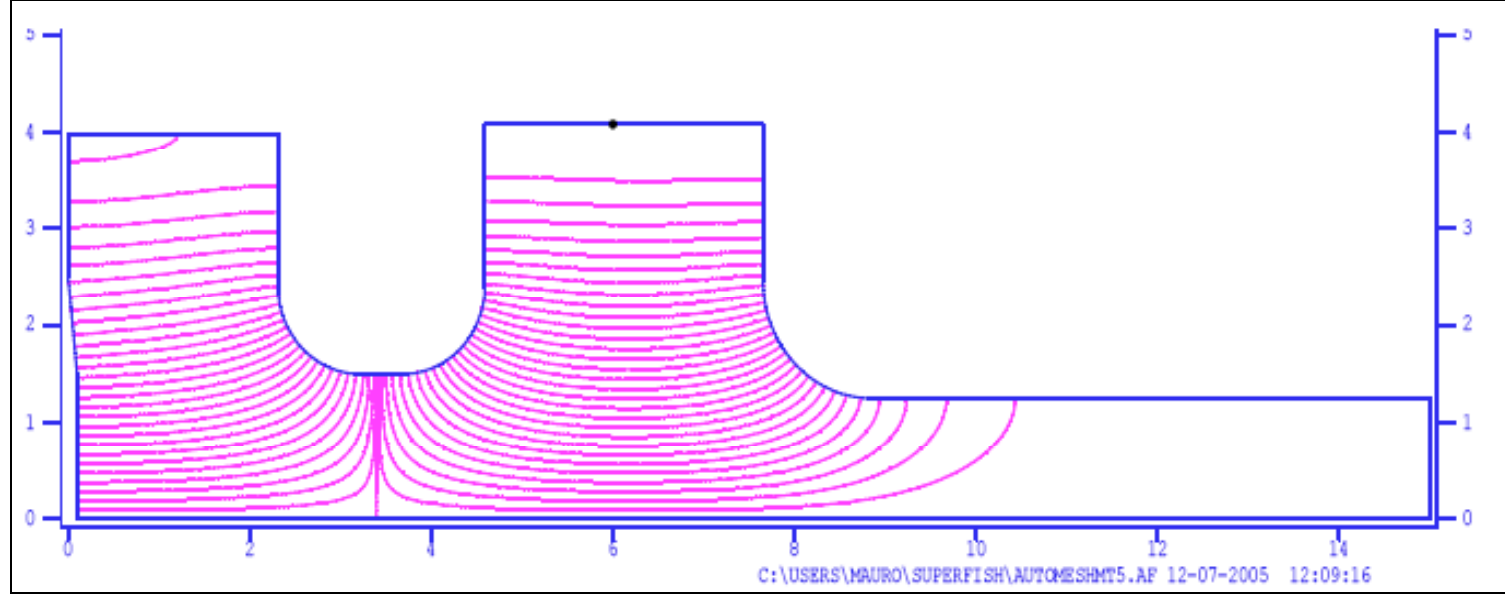


Figure 4.5: Case 5

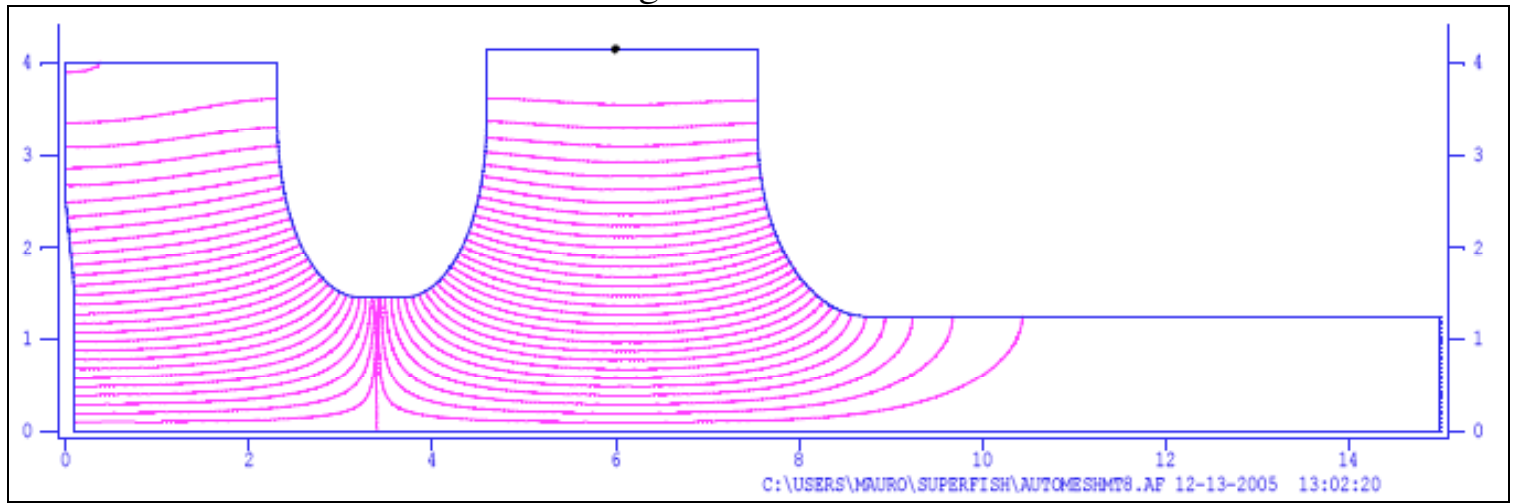

Different RF cavity geometries can satisfied the main requirement to be resonant at the frequency of 2997.9 MHz. We compare five cases. The field profiles along the axis are different, as shown in the Figure 4.6.

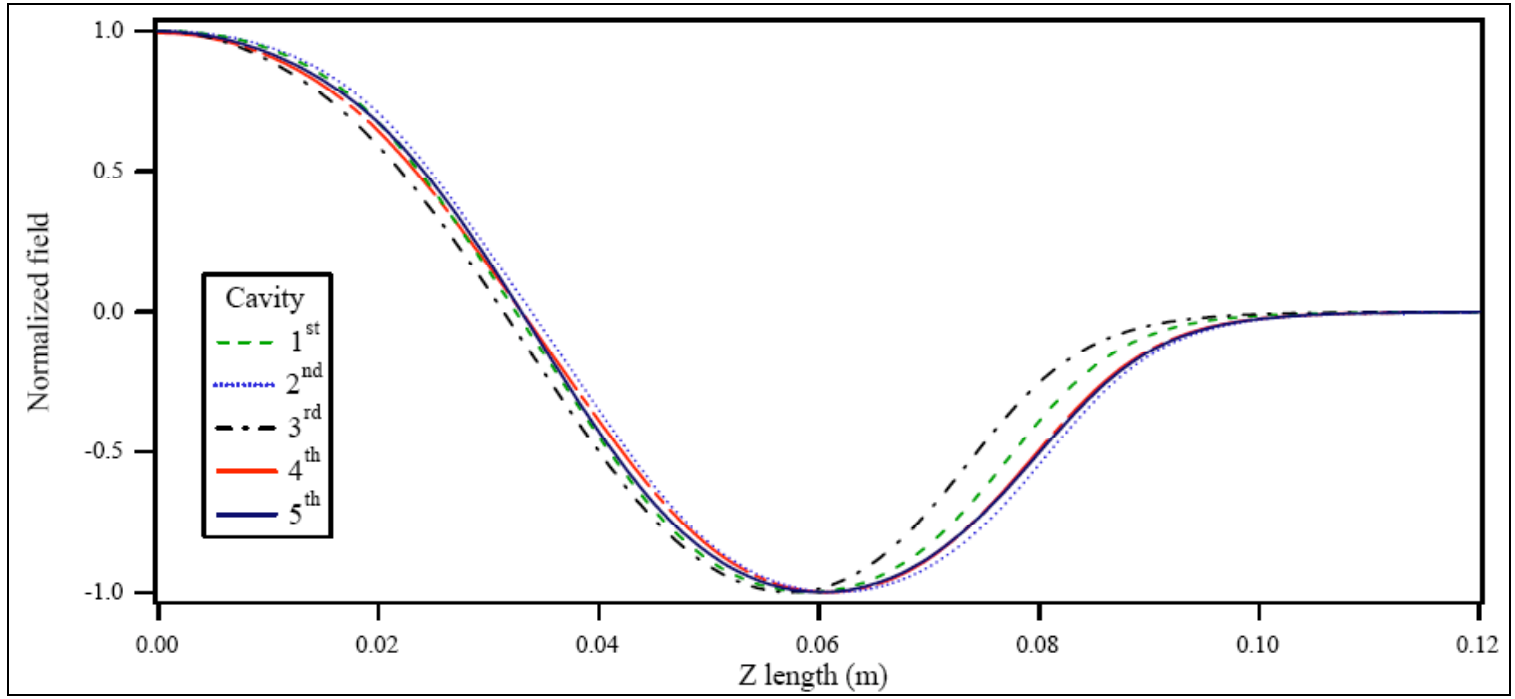

Figure 4.6: Axial electric field of the five cavities (pi-mode).

The cavities differ slightly in the parameters, but all of them are in an acceptable range. The main different between the analyzed cases is the launching phase that optimized the energy gain of the electron accelerated by the structure and this parameter plays an important role in the bunch beam dynamics.

\subsection{Field Balance and Mode Separation}

We examine the consequences of cavity geometry in some additional detail. In the pillbox cavity (Case 2) and reentrant cavity (Case 3) shown above, the cavity cells are redimensioned and optimized to produce a nearly 1:1 field balance in the pi-mode as well as a varying separation between the pi and zero mode resonant frequencies. We examine 
the effect on the shunt impedance, but do not yet optimize for maximum energy transfer efficiency between RF mode and the electron beam.

\section{Pillbox Cavity (Case 2)}

The nominal geometry for the RF gun is shown in Figure 4.7 below.

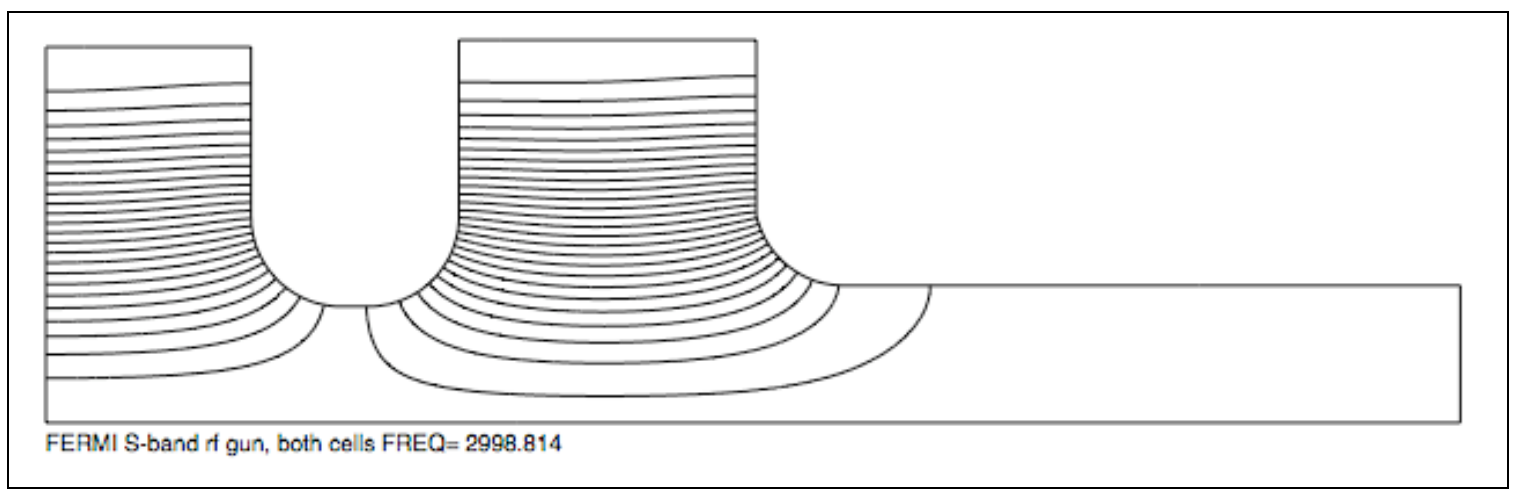

Figure 4.7: RF gun geometry from SUPERFISH model. Pi mode field lines are shown.

To produce various cavity tunes and frequency separations between the pi and zero modes, the radial dimensions of the cavity cell walls and connecting iris were adjusted. Increasing the frequency separation between the modes was implemented by increasing the coupling iris radius. The field balance for the pi mode was then re-established by independent adjustment of the two cell outer wall radii. Finally, the pi mode frequency was re-established by tandem (i.e. nearly equal) adjustment of the two cell outer radii. Table 4.1 lists the cavity geometries used to attain the various mode frequency separations.

Table 4.1: Cavity geometry variations and mode tunes (SUPERFISH).

\begin{tabular}{|c|c|c|c|c|c|}
\hline \multicolumn{3}{|c|}{ Radius [cm] } & \multicolumn{3}{c|}{ Tuning [all in MHz] } \\
\hline Half & Full & Iris & $\mathbf{f}_{\boldsymbol{\pi}}$ & $\mathbf{f}_{\mathbf{0}}$ & $\Delta \mathbf{f}$ \\
\hline 3.972 & 4.045 & 1.23 & 2998.830 & 2995.1 & 3.71 \\
\hline 3.977 & 4.048 & 1.25 & 2998.271 & 2993.5 & 4.80 \\
\hline 3.998 & 4.062 & 1.35 & 2997.876 & 2991.5 & 6.33 \\
\hline 4.020 & 4.070 & 1.45 & 2997.751 & 2998.4 & 9.33 \\
\hline 4.052 & 4.097 & 1.58 & 2997.515 & 2982.5 & 15.1 \\
\hline 4.089 & 4.121 & 1.73 & 2998.834 & 2975.1 & 23.7 \\
\hline
\end{tabular}

The on-axis electric field profiles for the pi and zero monopole modes are shown in Figure 4.8 below. For this study, only a rough field balance (to within $10 \%$ ) in the pi mode was required. Nevertheless, most of the geometries studied produced acceptable variations in the pi-mode field balance. The field balance in the zero mode does not exhibit much variation as the frequency separation increases. However, as can be seen in 
Figure 4.8, the field behavior in the coupling iris region between the two cells does show some trend toward higher fields as the mode separation increases.

This deviation from the pi-mode field distribution can exhibit several effects on the beam. The axial field null at the inter-cell iris has been 'filled in' by the presence of the zero mode. Hence, the purely defocusing electric field now contains a time dependent accelerating/decelerating component. The focusing properties of the cavity are then altered, and re-optimization of the beam dynamics is essential for adequate emittance compensation. From the standpoint of longitudinal dynamics, the zero mode can significantly alter the correlated energy spread, which again can affect the transverse beam quality due to chromaticity in the downstream focusing channels.
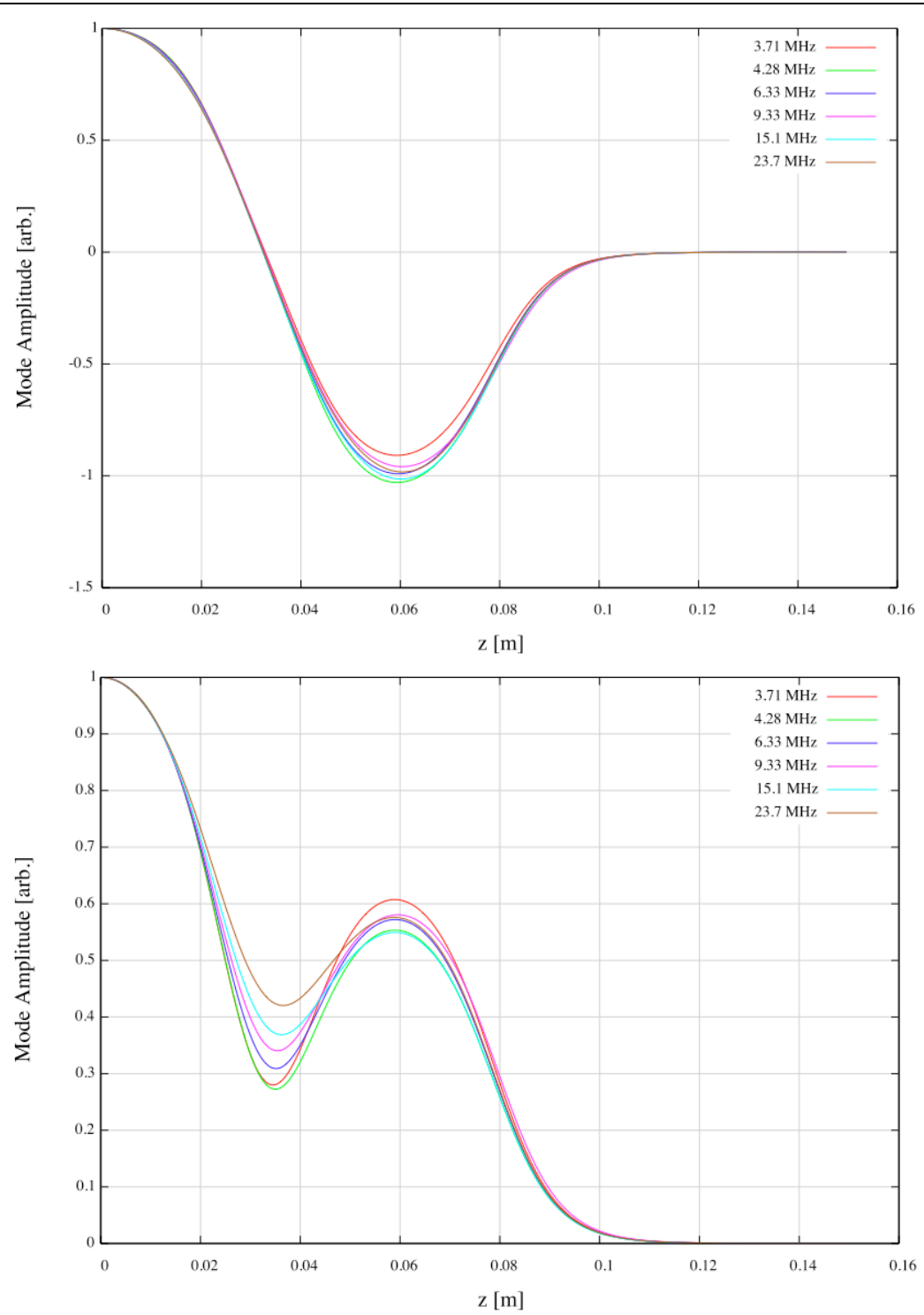

Figure 4.8: Axial electric field profiles for the pi mode (top) and zero mode (bottom), from SUPERFISH model. 
As a frequency domain model, SUPERFISH produces accurate values for the geometry dependent shunt impedance $([\mathrm{R} / \mathrm{Q}])$ and wall losses $\left(\mathrm{Q}_{\text {wall }}\right.$, from a perturbation calculation). These parameters are listed in Table 4.2 below. The wall losses remain fairly constant for each mode. The shunt impedances are seen to decrease with an increase in the mode frequency separation. This may be offset by re-optimizing the lengths of the two cells, which is described in the following sections.

Table 4.2: Resonant frequency monopole-mode characteristics

\begin{tabular}{|c|c|c|c|c|}
\hline & \multicolumn{2}{|c|}{ Pi mode } & \multicolumn{2}{c|}{ Zero mode } \\
\hline$\Delta \mathbf{f}[\mathbf{M H z}]$ & $\mathbf{Q}_{\text {wall }}$ & {$[\mathbf{R} / \mathbf{Q}][\mathbf{O h m}]$} & $\mathbf{Q}_{\text {wall }}$ & {$[\mathbf{R / Q}][\mathbf{O h m}]$} \\
\hline 3.71 & 13487 & 266 & 12584 & 5.47 \\
\hline 4.80 & 13690 & 259 & 12728 & 9.52 \\
\hline 6.33 & 13669 & 255 & 12813 & 8.23 \\
\hline 9.33 & 13806 & 252 & 12959 & 7.39 \\
\hline 15.1 & 13816 & 239 & 12873 & 10.7 \\
\hline 23.7 & 13857 & 232 & 13043 & 8.85 \\
\hline
\end{tabular}

\section{Re-entrant Cavity (Case 3)}

Adapting the existing design for an American S-band RF gun cavity geometry to European S-band frequencies preferentially requires that the half-cell length remain constant (at the dimensions optimized for $2856 \mathrm{MHz}$ resonant pi mode, in order to limit perturbations to the extensive half-cell instrumentation), but that the cell radii, iris radius, and full cell length may be varied to achieve the required frequency tuning and mode separation. Additionally, the RF transit angle across the cathode cell may be decreased by contouring the back plane of the cavity, creating a raised 'mesa' from which to launch the electron beam. Mode separation is investigated in this new geometry, and the full cell length is varied to maximize the cavity shunt impedance.

The nominal geometry for the RF gun is shown in Figure 4.9 below.

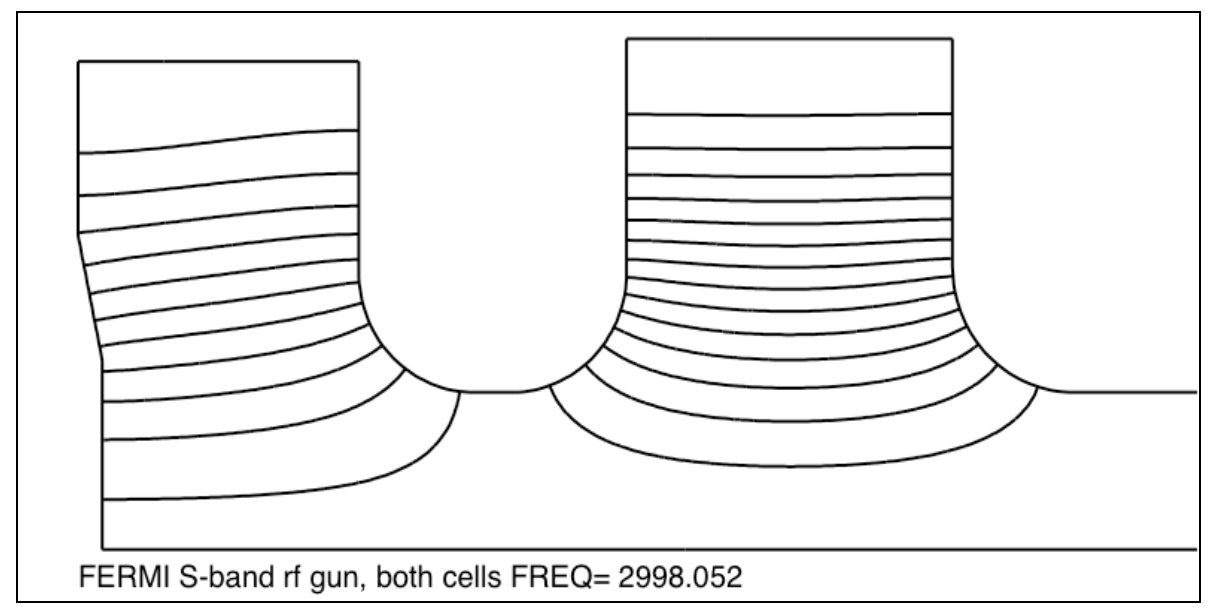


Figure 4.9: Reentrant RF gun geometry from SUPERFISH model. Pi mode field lines are shown.

To produce various cavity tunes and frequency separations between the pi and zero modes, the radial dimensions of the cavity cell walls and connecting iris were adjusted. Increasing the frequency separation between the modes was implemented by increasing the coupling iris radius. The field balance for the pi mode was then re-established by independent adjustment of the two cell outer wall radii. Finally, the pi mode frequency was re-established by tandem (i.e. nearly equal) adjustment of the two cell outer radii. Table 4.3 lists the cavity geometries used to attain the various mode frequency separations, calculated by SUPERFISH.

Table 4.3: Cavity geometry variations and mode tunes (SUPERFISH).

\begin{tabular}{|c|c|c|c|c|c|}
\hline \multicolumn{3}{|c|}{ Radius [cm] } & \multicolumn{3}{c|}{ Tuning [all in MHz] } \\
\hline Half-cell & Full-cell & Iris & $\mathbf{f}_{\boldsymbol{\pi}}$ & $\mathbf{f}_{\mathbf{0}}$ & $\boldsymbol{\Delta} \mathbf{f}$ \\
\hline 3.8853 & 4.06305 & 1.25 & 2998.05 & 2993.5 & 4.55 \\
\hline 3.9044 & 4.0766 & 1.35 & 2998.02 & 2990.9 & 7.12 \\
\hline 3.9386 & 4.10177 & 1.50 & 2997.9 & 2985.4 & 12.5 \\
\hline 3.9632 & 4.1196 & 1.60 & 2998.05 & 2980.46 & 17.6 \\
\hline
\end{tabular}

The on-axis electric field profiles for the pi and zero monopole modes are shown in Figures 4.10 and 4.11 below. For this study, only a rough field balance (to within a few percent accuracy) in the pi mode was required. The field balance in the zero mode does not exhibit much variation as the frequency separation increases. However, as can be seen in Figure 4.11, the field behavior in the coupling iris region between the two cells repeats the trend toward higher fields as the mode separation increases.

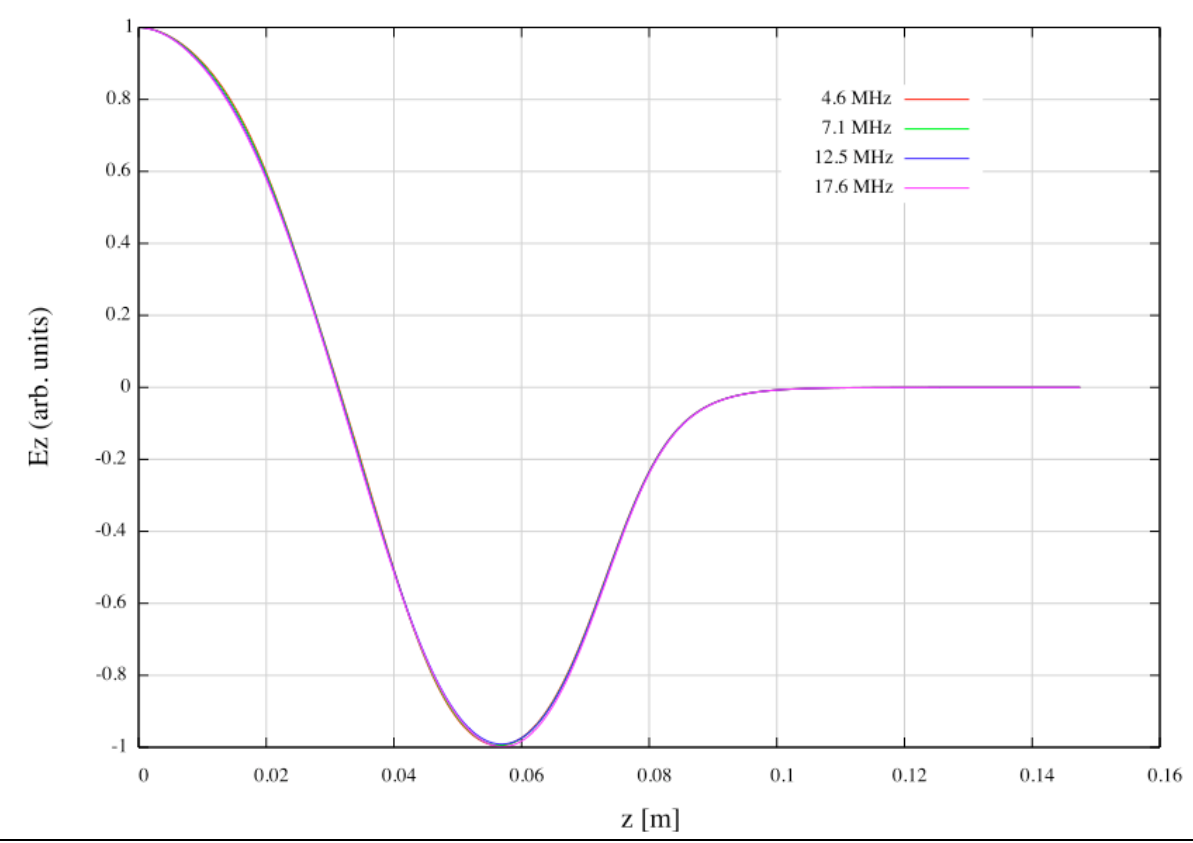


Figure 4.10: Axial electric field profiles for the pi mode, from SUPERFISH model.

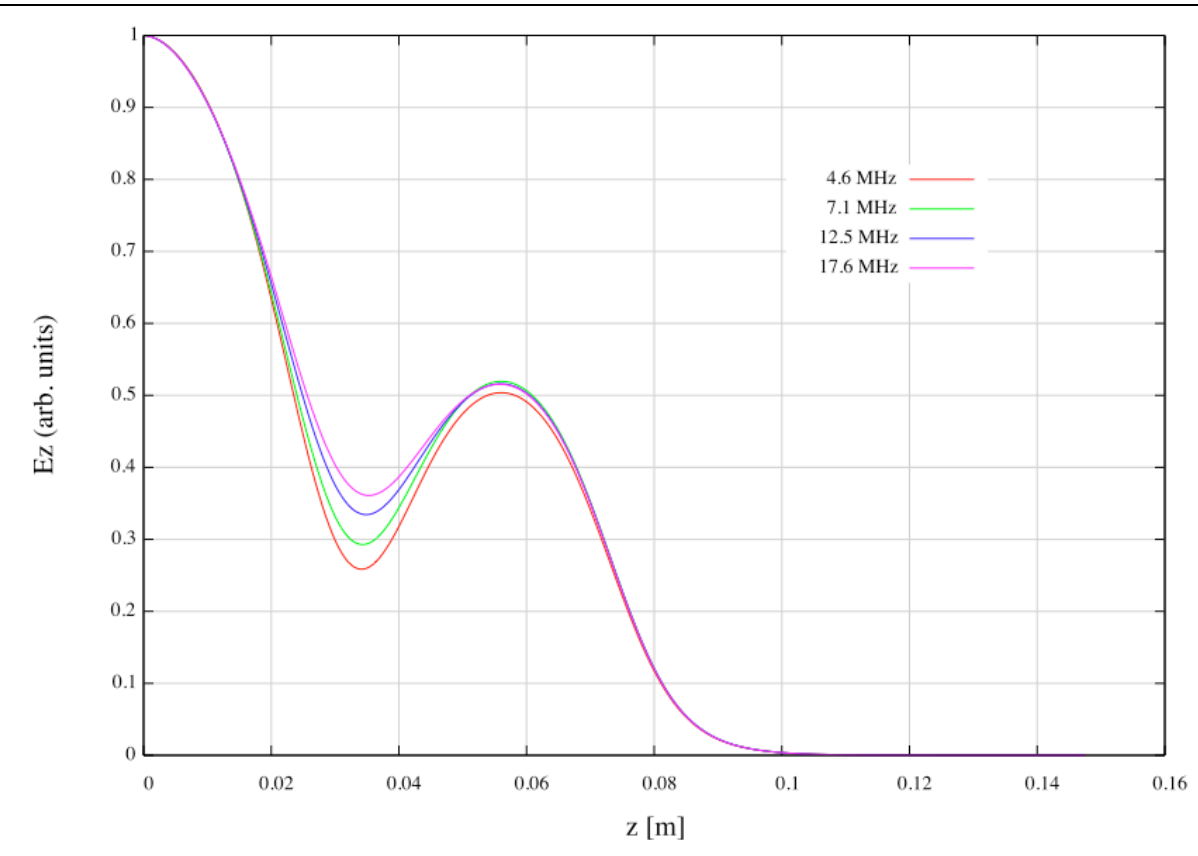

Figure 4.11: Axial electric field profiles for the zero mode, from SUPERFISH model.

In the following sections we examine the optimizations and response to the RF drive for the re-entrant pillbox cavity (Case 3).

\subsection{Shunt Impedance Optimization}

As a frequency domain model, SUPERFISH produces accurate values for the geometry dependent shunt impedance $([\mathrm{R} / \mathrm{Q}])$ and wall losses $\left(\mathrm{Q}_{\text {wall }}\right.$, from a perturbation calculation). These parameters are listed in Table 4.4 below. The transit-time factor (T) is calculated for constant velocity particles $\left(\beta_{\mathrm{z}}=1\right)$ via:

$$
T=\frac{\int_{\text {cavity length }} E_{z}\left(r=0, z^{\prime}\right) \sin \left(2 \pi z^{\prime} / \beta_{z} \lambda\right) d z^{\prime}}{\left|\int_{\text {cavity length }} E_{z}\left(r=0, z^{\prime}\right) d z^{\prime}\right|} .
$$


Table 4.4: Resonant frequency monopole-mode characteristics.

\begin{tabular}{|c|c|c|c|c|c|c|}
\hline & \multicolumn{3}{|c|}{ Pi mode } & \multicolumn{3}{c|}{ Zero mode } \\
\hline $\begin{array}{c}\Delta \mathbf{f} \\
{[\mathrm{MHz}]}\end{array}$ & $\mathbf{Q}_{\text {wall }}$ & $\mathbf{T}$ & $\begin{array}{c}{[\mathbf{R} / \mathbf{Q}]} \\
{[\mathbf{\Omega}]}\end{array}$ & $\mathbf{Q}_{\text {wall }}$ & $\mathbf{T}$ & $\begin{array}{c}{[\mathbf{R} / \mathbf{Q}]} \\
{[\mathbf{\Omega}]}\end{array}$ \\
\hline 4.55 & 12955 & 0.787 & 259 & 12422 & -0.175 & 14.4 \\
\hline 7.12 & 12997 & 0.787 & 252 & 12491 & -0.163 & 12.7 \\
\hline 12.5 & 13068 & 0.786 & 242 & 12572 & -0.165 & 13.2 \\
\hline 17.6 & 13130 & 0.785 & 235 & 12638 & -0.165 & 13.4 \\
\hline
\end{tabular}

The wall losses remain nearly constant for each mode. The shunt impedances are seen to decrease with an increase in the mode frequency separation, while the transit time factors remain essentially unchanged around the optimum for beam acceleration. In the absence of heavy beam loading, as is the case here, the efficiency of the cavities to transfer power from the RF drive to the beam in the fundamental mode is compromised by the increase in mode separation. This may be offset somewhat by re-optimizing the lengths of the full cell. Table 4.5 lists the results of re-optimizing the $17.6 \mathrm{MHz}$ frequency separation case for different lengths of the full-cell. The $9.3 \%$ loss in efficiency incurred by opening the cell-to-cell coupling iris and increasing the mode separation has been decreased to $6.6 \%$ by re-optimizing the full cell length. 
Table 4.5: Rf mode parameter dependence on full cell length.

\begin{tabular}{|c|c|c|c|c|c|c|c|c|c|}
\hline & & \multicolumn{3}{|c|}{ Pi mode } & \multicolumn{3}{c|}{ Zero mode } \\
\hline Case & $\begin{array}{c}\Delta \mathbf{L}_{\text {full }} \\
(\mathbf{c m})\end{array}$ & $\begin{array}{c}\text { Freq. } \\
{[\mathbf{M H z}]}\end{array}$ & $\mathbf{T}$ & $\mathbf{Q}_{\text {wall }}$ & $\begin{array}{c}{[\mathbf{R} / \mathbf{Q}]} \\
{[\mathbf{\Omega}]}\end{array}$ & $\begin{array}{c}\text { Freq. } \\
{[\mathbf{M H z}]}\end{array}$ & $\mathbf{T}$ & $\mathbf{Q}_{\text {wall }}$ & $\begin{array}{c}{[\mathbf{R} / \mathbf{Q}]} \\
{[\mathbf{\Omega}]}\end{array}$ \\
\hline $\mathrm{F}$ & -0.05 & 2998.25 & 0.786 & 13031 & 236 & 2980.55 & -0.167 & 12596 & 13.7 \\
\hline $\mathrm{A}$ & 0 & 2998.05 & 0.785 & 13130 & 235 & 2980.46 & -0.165 & 12639 & 13.4 \\
\hline $\mathrm{B}$ & +0.05 & 2998.09 & 0.783 & 13233 & 237 & 2980.57 & -0.166 & 12676 & 13.5 \\
\hline $\mathrm{C}$ & +0.10 & 2997.90 & 0.782 & 13311 & 240 & 2980.59 & -0.157 & 12731 & 12.3 \\
\hline $\mathrm{E}$ & +0.15 & 2997.90 & 0.781 & 13395 & 242 & 2980.76 & -0.152 & 12779 & 11.7 \\
\hline $\mathrm{D}$ & +0.20 & 2997.99 & 0.778 & 13505 & 243 & 2980.83 & -0.157 & 12800 & 12.5 \\
\hline
\end{tabular}




\section{Launching with $\beta=0$ and effective $[R / Q]$}

In the case of a photoinjector source, the optimizations performed above contain a serious error. The beam is emitted from the photocathode with nearly zero forward momentum and requires several centimeters of acceleration in the RF cavity fields to attain a velocity $\beta \sim$ c. Net energy transfer from the RF field to the beam is complicated by slippage effects.

To estimate the effective shunt impedance of the cavity as seen by the electron beam, we calculate the single particle energy gain $(\Delta \mathrm{W})$ as a function of launch phase. For an electron with negligible kinetic energy at emission, the system of equations is solved:

$$
\begin{aligned}
& \frac{d t}{d z}=\frac{1}{\beta c} \\
& \frac{d W}{d z}=e E_{\pi}(z) \cos \left(\omega_{\pi} t+\varphi_{\pi}\right)
\end{aligned}
$$

Here, we express the spatial profile of the pi-mode as $\mathrm{E}_{\pi}$, the pi-mode angular frequency as $\omega_{\pi}$, and the temporal phase at emission as $\varphi_{\pi}$. The particle velocity is calculated as

$$
\beta=\sqrt{1-\frac{1}{\left(1+\frac{W}{m c^{2}}\right)^{2}}}
$$

This system of equations is solved, tracking the energy and time-of-flight of a reference electron from emission $(t=0, \beta=0)$ to the exit of the RF gun, for a range of initial launch phases, assuming a peak axial electric field gradient at the cathode of $110 \mathrm{MV} / \mathrm{m}$. The effective shunt impedance is then calculated by

$$
\left[\frac{R}{Q}\right]_{e f f}=\frac{\left(\Delta W_{\max } / e\right)^{2}}{\omega_{\pi} U_{\pi}}
$$

where $U_{\pi}$ is the energy stored in the cavity. This quantity is mode dependent and is calculated by SUPERFISH. In general, the definition of the effective shunt impedance is launch phase dependent. Here, we have introduced the convention of utilizing the maximum energy gain at the cavity exit in the definition of $[R / Q]_{\text {eff }}$ as a means to label the launch phase. Table 4.6 lists the characteristics and effective shunt impedances for the various geometries that produce a $17.6 \mathrm{MHz}$ pi-zero mode frequency separation. The change in energy ( $\Delta$ Energy) is the difference between the maximum particle energy within the structure and the particle energy upon exit. Comparing the values of the shunt impedances between Tables 4.5 and 4.6, we see that for this class of RF gun and electric field gradient, the effective shunt impedance loss due to particle slippage at low $\beta$ is in 
the range of $2 \%-8 \%$ compared to the $\beta=1$ model. The $[R / Q]_{\text {eff }}$ displays a weak dependence with variations in geometry. We may then choose to optimize the cavity geometry with exit beam energy (Case $\mathrm{D}$ in Table 4.6 below). 
Table 4.6: Beam energy dependence, $\pi$ mode only, $17.6 \mathrm{MHz}$ separation, $110 \mathrm{MV} / \mathrm{m}$.

\begin{tabular}{|c|c|c|c|c|c|c|c|c|}
\hline Case & $\Delta \mathrm{L}_{\text {full }}[\mathrm{cm}]$ & $\begin{array}{c}\text { Max Energy }{ }^{1} \\
{[\mathrm{MeV}]}\end{array}$ & Phase $^{1}\left[{ }^{\circ}\right]$ & $\begin{array}{c}\text { Max Exit } \\
\text { Energy }^{2} \\
{[\mathrm{MeV}]}\end{array}$ & Phase $^{2}\left[^{\circ}\right]$ & $\Delta \mathrm{E}[\mathrm{keV}]$ & $\mathbf{Q}_{\text {wall }}$ & $\begin{array}{c}{[R / Q]_{e f f}} \\
{[\Omega]}\end{array}$ \\
\hline $\mathrm{F}$ & -0.05 & 4.68 & -50 & 4.65 & -50 & -28 & 13031 & 224.1 \\
\hline A & 0 & 4.71 & -50 & 4.68 & -50 & -30 & 13130 & 225.6 \\
\hline$B$ & +0.05 & 4.75 & -50 & 4.72 & -55 & -32 & 13233 & 226.8 \\
\hline C & +0.10 & 4.72 & -50 & 4.69 & -55 & -31 & 13311 & 229.2 \\
\hline$E$ & +0.15 & 4.72 & -55 & 4.69 & -55 & -32 & 13395 & 236.5 \\
\hline $\mathrm{D}$ & +0.20 & 4.80 & -55 & 4.76 & -55 & -35 & 13505 & 224.5 \\
\hline
\end{tabular}


Figure 4.12 displays the launch phase dependence on the maximum energy and exit energy of the reference particle. Figure 4.13 shows the resulting evolution of the particle's kinetic energy from launch to the cavity exit. We see that the maximum particle energy actually occurs $\sim 1 \mathrm{~cm}$ from the cavity exit, though the difference between these energies is insignificant.

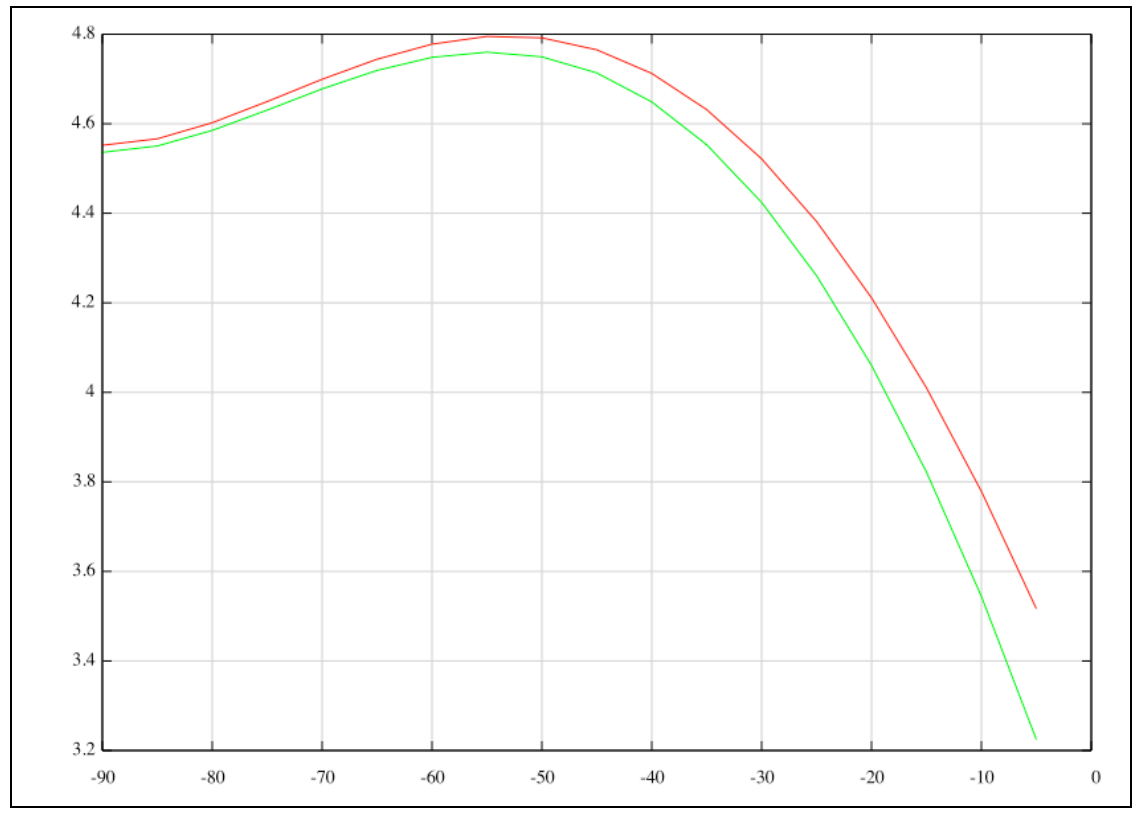

Figure 4.12: Maximum (red) and exit (green) beam energy (in MeV) versus launch RF phase (in degrees of $2.998 \mathrm{GHz}$ ). (Table 4.6, Case D)

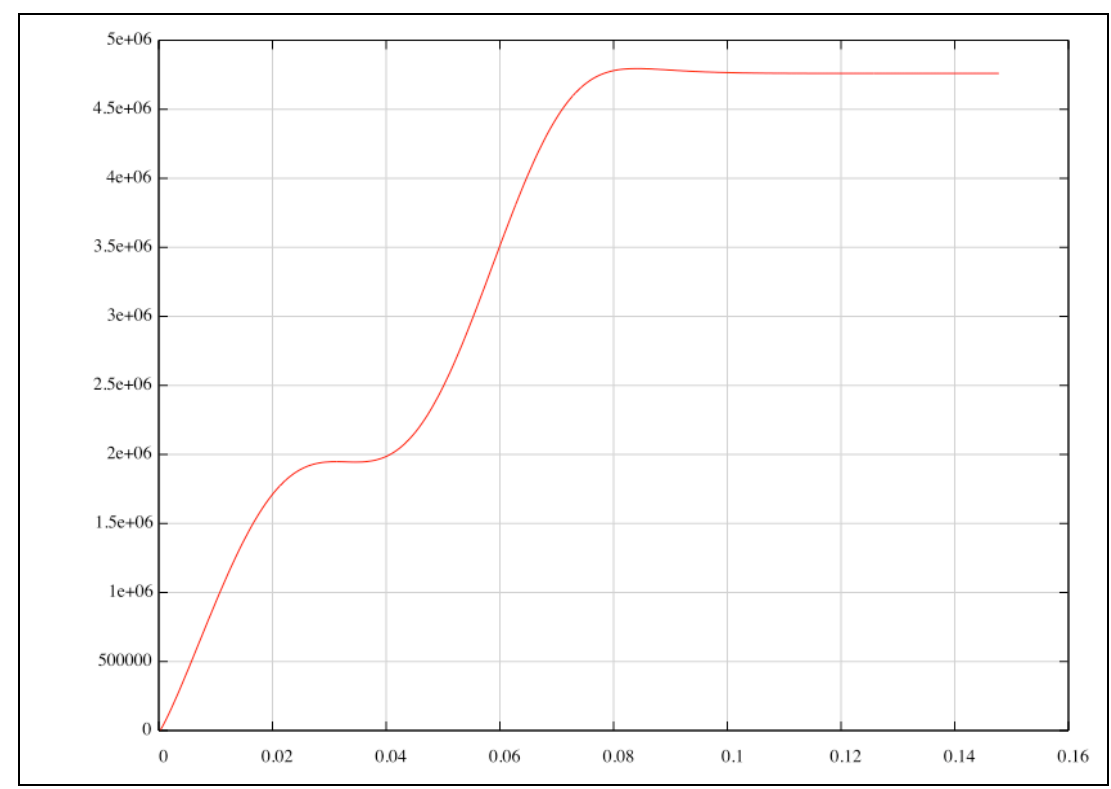

Figure 4.13: Beam energy (in eV) versus longitudinal position (in $\mathrm{m}$ ) for optimal launch phase. (Table 4.6, Case D, $-55^{\circ}$ ) 
Returning to the comparison of cavity geometries with varying pi-zero mode frequency separation, we calculate the effective shunt impedances seen by the beam. The results are shown in Table 4.7 below. Including emission and beam lethargy effects (i.e. slippage), we see that only $\sim 4 \%$ reduction in the shunt impedance results. Moreover, we see that increasing the mode frequency separation from a nominal value of $\sim 4.6 \mathrm{MHz}$ to nearly 18 $\mathrm{MHz}$ results in a reduction of $\sim 10 \%$ to the efficiency of transferring RF power from the source to the electron beam. 
Table 4.7: Variation of effective [R/Q] with mode frequency separation.

\begin{tabular}{|c|c|c|c|c|c|c|}
\hline $\begin{array}{c}\Delta \mathbf{f} \\
{[\mathrm{MHz}]}\end{array}$ & $\begin{array}{c}\text { Max } \\
\text { Energy } \\
{[\mathrm{MeV}]}\end{array}$ & $\begin{array}{c}\text { Phase } \\
{\left[{ }^{\circ}\right]}\end{array}$ & $\begin{array}{c}\text { Max Exit } \\
\text { Energy } \\
{[\mathrm{MeV}]}\end{array}$ & $\begin{array}{c}\text { Phase } \\
{\left[{ }^{\circ}\right]}\end{array}$ & $\begin{array}{c}{[R / Q]_{\text {eff }}} \\
{[\Omega]}\end{array}$ & $\begin{array}{c}\left.[R / Q]_{(\beta)}=1\right) \\
{[\Omega]}\end{array}$ \\
\hline 4.6 & 4.7199 & -50 & 4.6905 & -50 & 248.4 & 259 \\
\hline 7.12 & 4.7136 & -50 & 4.6840 & -50 & 241.9 & 252 \\
\hline 12.5 & 4.6944 & -50 & 4.6646 & -55 & 232.3 & 242 \\
\hline 17.6 & 4.7708 & -50 & 4.7372 & -55 & 224.5 & 235 \\
\hline
\end{tabular}




\subsection{Power Coupling}

A three-dimensional model of the basic pillbox RF gun structure (Case 2) was created and implemented using Microwave Studio [4.3] (Figure 4.14 below). The cavity is modeled with symmetric waveguides and ports, with full height WR-284 waveguide extending to with $3 \mathrm{~mm}$ of the outer cavity walls and a simple, rectangular opening providing the waveguide-cavity coupling iris. The dimensions of the coupling aperture were varied until the time-domain response at the smallest mode frequency separation roughly corresponded to the values measured at the SLAC Gun Test Facility [4.4]. The coupling aperture dimensions were then fixed, as the cell and on-axis iris radii were adjusted to vary the pi and zero mode frequency separation.

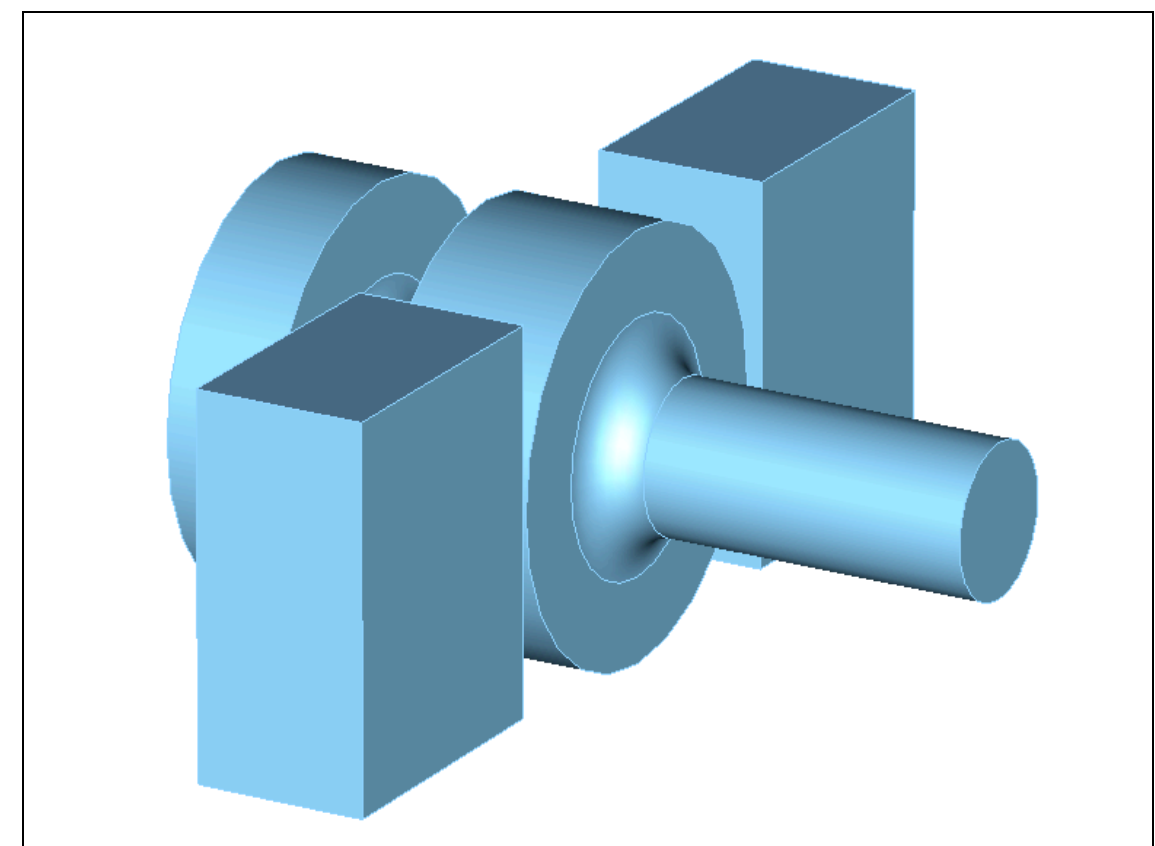

Figure 4.14: Microwave Studio model for the RF gun with dual waveguide feeds.

\section{Frequency-domain}


Table 4.8: Frequency-domain results for various pi/zero mode tunings.

\begin{tabular}{|c|c|c|c|c|c|c|c|}
\hline \multicolumn{3}{|c|}{ Initial Radius [cm] } & \multicolumn{5}{|c|}{ Final Tuning [frequencies in MHz] } \\
\hline Half & Full & Iris & $\mathbf{f}_{\boldsymbol{\pi}}$ & $\mathbf{Q}_{\text {wall, } \boldsymbol{\pi}}$ & $\mathbf{f}_{\mathbf{0}}$ & $\mathbf{Q}_{\text {wall, }}$ & $\mathbf{\Delta f}$ \\
\hline 3.972 & 4.045 & 1.23 & 2998.094 & 12478 & 2994.340 & 12116 & 3.75 \\
\hline 4.020 & 4.070 & 1.45 & 2997.890 & 12902 & 2987.600 & 12419 & 10.29 \\
\hline 4.052 & 4.097 & 1.58 & 2998.540 & 13041 & 2982.860 & 12600 & 15.68 \\
\hline
\end{tabular}


The cell geometries were adjusted to produce various pi-zero mode resonant frequency separation. The results of frequency-domain simulations are listed in Table 4.8 below. The axial electric field profiles are shown in Figure 4.15. These results agree well with the SUPERFISH models.

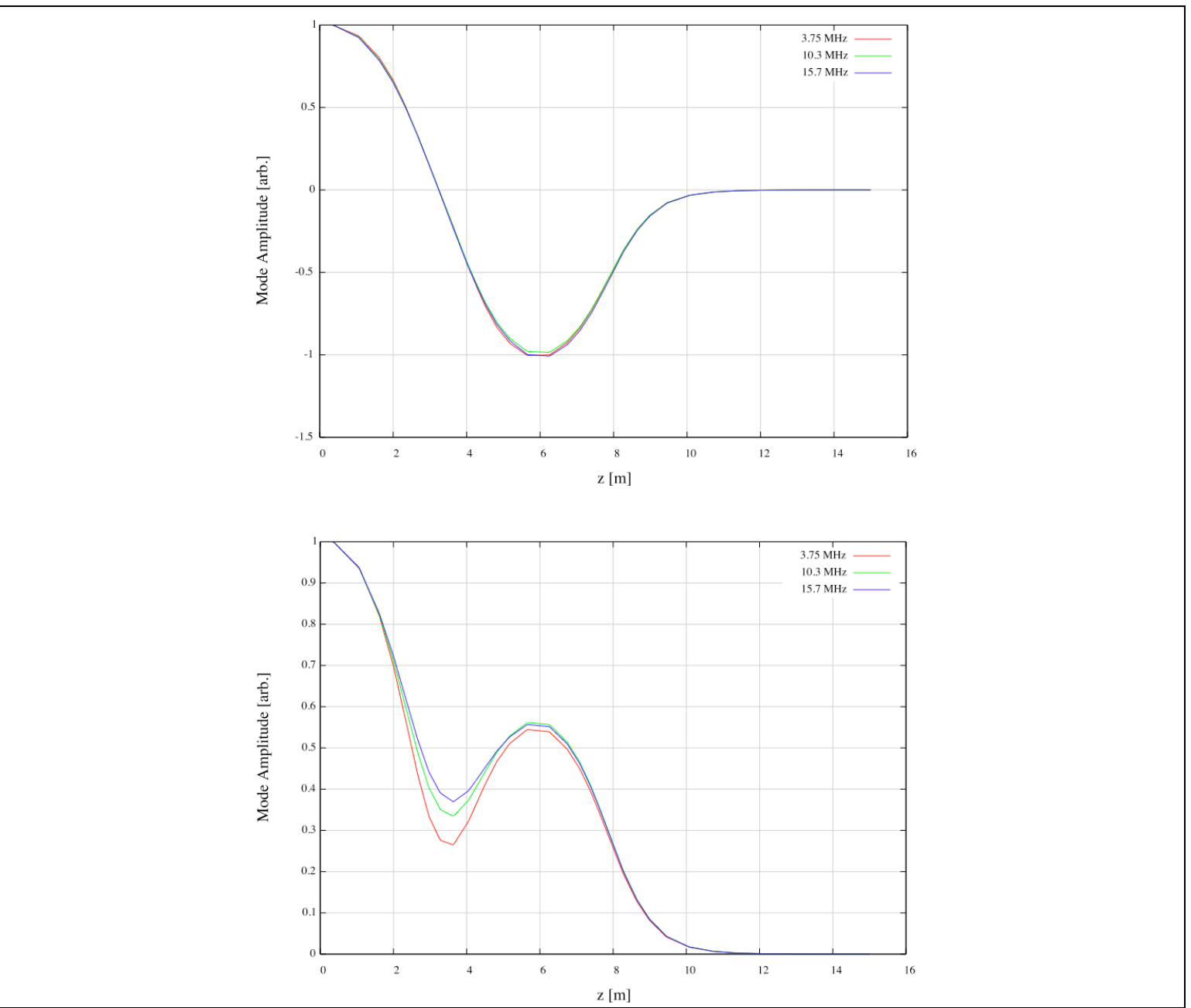

Figure 4.15: Axial electric field profiles for the pi mode (top) and zero mode (bottom), from frequency-domain Microwave Studio model.

\section{Time domain}

Time-domain calculations were performed in Microwave Studio to calculate the waveguide coupling factors $(\beta)$ for the pi and zero modes at their resonant frequencies. The waveguides were tandemly excited in the $\mathrm{TE}_{10}$ mode with a flat-top signal in the frequency domain with limits between $2980 \mathrm{MHz}$ and $3010 \mathrm{MHz}$. The reflected signals were then Fourier analyzed, and the complex reflection coefficients calculated. These are shown in Figure 4.16. The larger radius curve is the pi mode response and the smaller 
radius curve is the zero mode response. This plot shows us that the pi mode is overcoupled $(\beta>1)$ since the response curve encloses the origin, while the zero mode is undercoupled $(\beta<1)$ since the origin is excluded. For each of the cases studied, this behavior was seen to be generic.

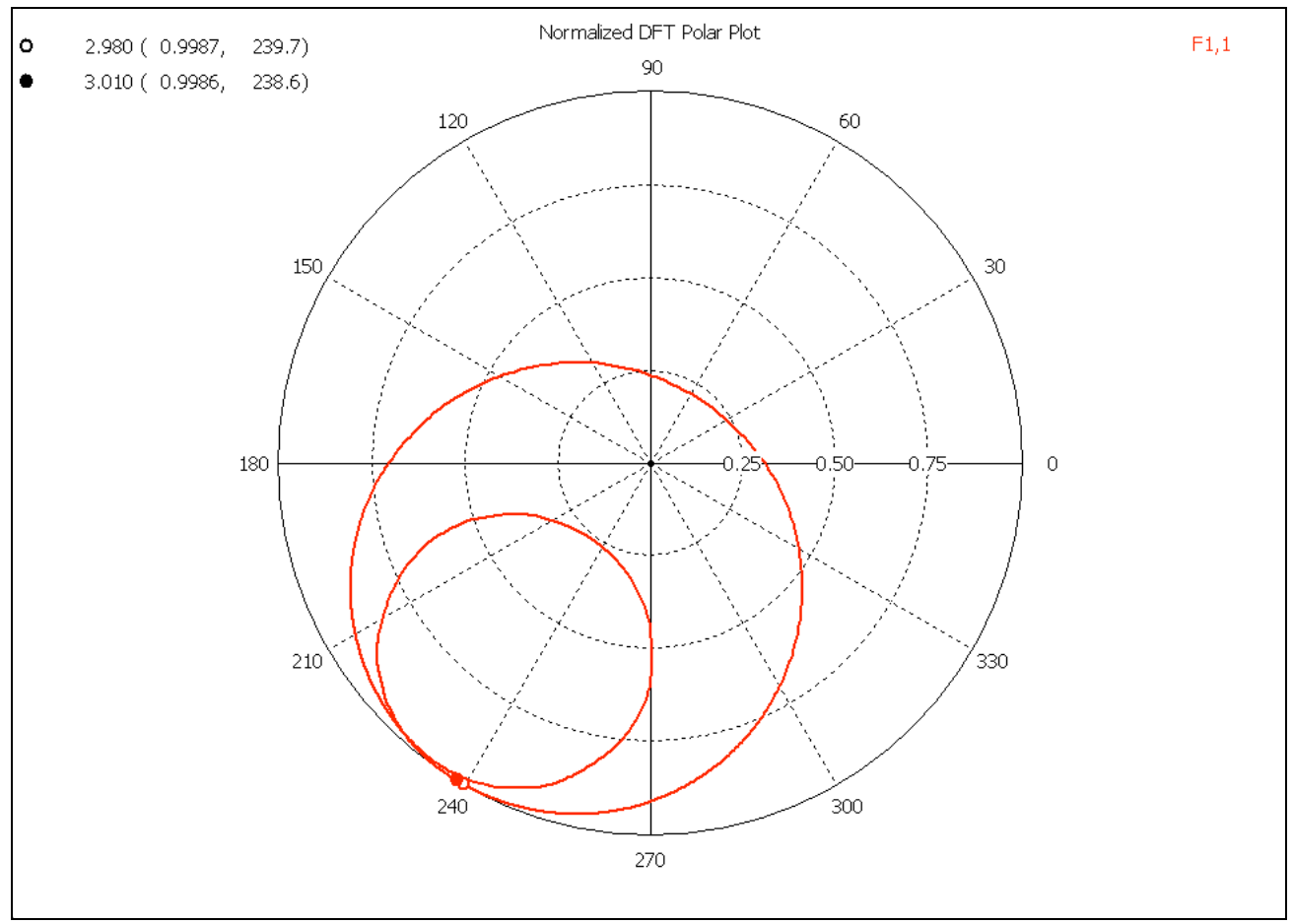

Figure 4.16: Complex reflection coefficient $(\rho)$ measurement.

The voltage standing wave ratio (VSWR) is calculated from the reflection coefficient $(\rho)$ :

$$
V S W R=\frac{1+|\rho|}{1-|\rho|}
$$

In the overcoupled case the coupling parameter $\beta=\mathrm{VSWR}$, while in the undercoupled case $\beta=1 /$ VSWR. The results of the time-domain modeling are listed in Table 4.9. Without re-optimization of the input waveguide coupler, the pi mode is well overcoupled, while the zero mode can approach critical coupling. Even after optimization of the coupling to the pi mode, we will expect the zero mode coupling to be a factor of 2-3 smaller.

Table 4.9: Time-domain results for the waveguide coupling parameters.

\begin{tabular}{|c|c|c|c|c|}
\hline & \multicolumn{2}{|c|}{ Pi mode } & \multicolumn{2}{c|}{ Zero mode } \\
\hline$\Delta \mathbf{f}[\mathbf{M H z}]$ & VSWR & $\boldsymbol{\beta}=\mathbf{V S W R}$ & VSWR & $\boldsymbol{\beta}=\tilde{1 / V S W R}$ \\
\hline 3.75 & 1.558 & 1.558 & 1.701 & 0.588 \\
\hline 10.29 & 1.918 & 1.918 & 1.131 & 0.884 \\
\hline 15.68 & 1.887 & 1.887 & 1.220 & 0.820 \\
\hline
\end{tabular}




\subsection{Klystron pulse shape and transient response}

The transient response of the two-mode RF gun structure has been analyzed under a series of progressively complicated (and realistic) excitation signals.

\section{Klystron Pulse Model and Excitation Spectrum}

A simple model of the klystron pulse that drives the cavity modes has been spectrally analyzed. The input pulse envelope is assumed to have a $3 \mu$ sec flat-top with linear, 100ns rise- and fall- times, with single carrier-frequency modulation at $2998 \mathrm{MHz}$. The spectrum of the amplitude and relative phase of the drive pulse have been calculated and are shown in Figure 4.17, and tabulated in Table 4.10.

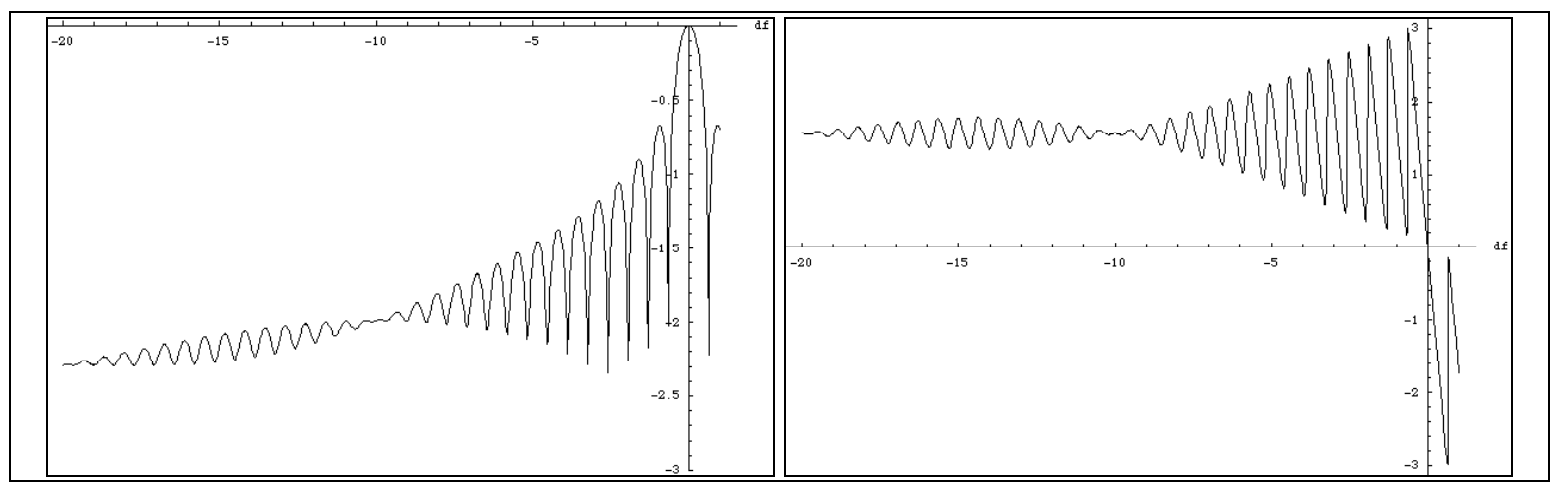

Figure 4.17: Log-amplitude (left) and relative phase (right) of the klystron voltage pulse frequency components, normalized to the $2998 \mathrm{MHz}$ component, versus frequency difference (in $\mathrm{MHz}$ ).

Table 4.10: Relative amplitude and phase at several frequency separations from the carrier frequency.

\begin{tabular}{|r|c|c|c|c|c|c|}
\hline$\Delta \mathbf{f}(\mathbf{M H z})$ & 0 & -3.5 & -5 & -10 & -15 & -20 \\
\hline $\begin{array}{r}\text { Relative } \\
\text { amplitude }\end{array}$ & 1. & 0.052 & 0.024 & 0.010 & 0.007 & 0.005 \\
\hline $\begin{array}{r}\text { Relative } \\
\text { phase ( }\end{array}$ & 0. & 77.94 & 122.5 & 90. & 102.0 & 90. \\
\hline
\end{tabular}

At large offsets from the carrier frequency and at steady-state, the relative amplitude is seen to decrease exponentially, while the relative phase oscillates about $90^{\circ}$. Hence, a rough expectation is that, at steady-state, the pi mode will track the phase and amplitude generated by the component at $2998 \mathrm{MHz}$, while the zero mode will track with the amplitude and phase shown above. This estimate does not account for differences in coupling to the klystron pulse for the two modes, nor for mode quality factor differences that determine the actual transient response. We consider those effects next. 


\subsection{Two-mode resonant cavity response under ideal RF drive}

The behavior of the RF gun structure under non-trivial RF drive waveforms is determined from analysis of the equivalent circuit equation description. The transient and steady state response of the cavity modes are determined and related to measured values of the fields in the full- and half-cells. In this section, the RF drive waveform is assumed to take an idealized, though nontrivial form. However, we will assume for now that the phase of the RF drive signal remains constant over the pulse.

\section{Equivalent circuit equation}

We derive an expression for the cavity voltages following [4.5]. The time dependence of the voltage in an oscillating resonant mode with coupling to finite conductivity walls and an external waveguide is expressed as

$$
\left(\frac{d^{2}}{d t^{2}}+\omega_{0}^{2}\right) V_{0}=-\frac{\omega_{0}}{Q_{w}} \frac{d}{d t} V_{0}+\frac{\omega_{0}}{Q_{e x t}} \frac{d}{d t}\left(V_{F}-V_{R}\right)
$$

In this expression we express the voltages in terms of those observed within the cavity. The forward and reverse voltages within this impedance normalization are related to the observed voltages within the waveguide $\left(V_{ \pm}\right)$by

$$
V_{F, R}=n V_{ \pm}
$$

where

$$
n=\left[\frac{R_{s}}{2 Z_{c} \beta}\right]^{1 / 2}
$$

is the so-called 'transformer ratio', which follows from power balance at steady-state. This links the observed voltages to the cavity shunt impedance $\left(R_{s}\right)$, the waveguide-cavity mode coupling parameter $\left(\beta=Q_{w} / Q_{\text {ext }}\right)$, and the characteristic impedance of the traveling waveguide mode $\left(Z_{c}\right)$. Continuity of the transverse electric fields at the waveguide boundary is guaranteed by enforcing the boundary condition

$$
V_{0}=V_{F}+V_{R}
$$

Using this condition, we rewrite Eqn. (4.6) as

$$
\left(\frac{d^{2}}{d t^{2}}+\frac{\omega_{0}}{Q_{L}} \frac{d}{d t}+\omega_{0}^{2}\right) V_{0}=\frac{2 \omega_{0}}{Q_{e x t}} \frac{d}{d t} V_{F}=\frac{2 n \omega_{0}}{Q_{e x t}} \frac{d}{d t} V_{+}
$$

where we use the usual expression for loaded quality factor in the mode 


$$
\frac{1}{Q_{L}}=\frac{1}{Q_{0}}+\frac{1}{Q_{e x t}}
$$

We note that the transformer ratio, shunt impedance, and coupling parameter are dependent on the particular waveguide and cavity mode distributions. In our case here, we assume a single waveguide mode $\left(\mathrm{TE}_{01}\right)$ that couples to our two TM cavity modes.

For the two cavity modes $(0$ and $\pi)$ present within the bandwidth of the RF drive, we assume that the two modes are independently coupled. This assumption is valid if the frequency separation between the two modes is larger than width of each resonance. In this approximation, we rewrite Eqn. (4.10) for the two modes as

$$
\begin{aligned}
& \left(\frac{d^{2}}{d t^{2}}+\frac{\omega_{0}}{Q_{L, 0}} \frac{d}{d t}+\omega_{0}^{2}\right) V_{0}=\frac{2 n_{0} \omega_{0}}{Q_{e x t, 0}} \frac{d}{d t} V_{+, 0}, \\
& \left(\frac{d^{2}}{d t^{2}}+\frac{\omega_{\pi}}{Q_{L, \pi}} \frac{d}{d t}+\omega_{\pi}^{2}\right) V_{\pi}=\frac{2 n_{\pi} \omega_{\pi}}{Q_{e x t, \pi}} \frac{d}{d t} V_{+, \pi} .
\end{aligned}
$$

\section{Solution under constant phase approximation}

We seek a time domain solution to Eqns. $(4.12 \mathrm{a}, \mathrm{b})$ that allows us to investigate both transient and steady-state behavior. The forward voltage waveform is assumed to take the form

$$
V_{+}(t)=A(t) \cos \Phi_{+}(t)=\Re A(t) \exp \left[-i \Phi_{+}(t)\right],
$$

where $\mathfrak{R}$ indicates the real part of a complex value. The amplitude, $A(t)$, and phase, $\Phi_{+}(t)$, of the waveform are real-valued. The phase dependence is assumed to contain a constant carrier frequency, $\omega$, and a modulated phase offset, i.e. $\Phi_{+}(t)=\omega t+\phi_{+}(t)$. In this note, we will assume that the phase modulation is negligible compared to $2 \pi$, so that $\Phi_{+}(t) \approx \omega t$.

We will work with complex phasors in the rotating frame defined by the drive frequency, $\omega, V(t)=\Re \tilde{V}(t) e^{-i \omega t}$. We also use the slowly varying envelope approximation, $\dot{\tilde{V}}<<\omega V$, where the overdot signifies the total time derivative (i.e. $d / d t$ ). Neglecting second order envelope derivative terms, we re-express Eqns. (4.12a,b) as

$$
\dot{\tilde{V}}_{0}+\frac{\omega_{0}}{2 Q_{L, 0}}\left(1+i \tan \psi_{0}\right) \tilde{V}_{0}=\frac{n_{0}}{Q_{e x t, 0}}\left(\omega \tilde{V}_{+}+i \dot{\tilde{V}}_{+}\right)
$$




$$
\dot{\tilde{V}}_{\pi}+\frac{\omega_{\pi}}{2 Q_{L, \pi}}\left(1+i \tan \psi_{\pi}\right) \tilde{V}_{\pi}=\frac{n_{\pi}}{Q_{e x t, \pi}}\left(\omega \tilde{V}_{+}+i \dot{\tilde{V}}_{+}\right) .
$$

We have defined the tuning angles, $\psi_{(0, \pi)}$, by

$$
\tan \psi_{(0, \pi)}=2 Q_{L,(0, \pi)}\left(\frac{\omega_{(0, \pi)}-\omega}{\omega_{(0, \pi)}}\right) .
$$

\section{Input voltage waveform}

We first analyze the simple, idealized case of an analytically defined amplitude waveform given by

$$
V_{F}(t) / V_{F 0}=H(t)\left(1-e^{-t / \tau}\right)-H(t-T)\left(1-e^{-(t-T) / \tau}\right),
$$

where $V_{F O}$ is the peak voltage amplitude of the input signal, $H(t)$ is the Heaviside unit step function, $\tau$ is an exponential rise/fall time, and $\mathrm{T}$ defines the time at which the flat top portion begins to decay. The pulse has zero amplitude for times $t<0$, a $10 \%-90 \%$ risetime of $\sim 2.2 \tau$, and a flat top interval stretching out to $t=T$. Since the signal phase modulation is negligible, we identify the forward voltage phasor with the amplitude waveform, $\tilde{V}_{+}=V_{F}$.

We seek a solution to the equations of motions (Eqns. 4.14a,b) via Laplace transform techniques. Standard mathematical tables [4.6] are used to generate the particular transform pairs we need to solve the set of equations, subject to the initial conditions $\tilde{V}_{+}(0)=\tilde{V}_{0}(0)=\tilde{V}_{\pi}(0)=0$. Foregoing a tedious display of algebra, the result is the time response for the two modes:

$$
\begin{aligned}
& \tilde{V}_{0}(t),\left(V_{F 0} n_{0} \frac{2 \beta_{0}}{1+\beta_{0}}\right)=\cos \psi_{0} e^{-i \psi_{0}} \times \\
& \left(\left\{1-e^{-\left(\frac{1+i \tan \psi_{0}}{2 Q_{L, 0}}\right) \omega t}+(\omega \tau-i)\left[\frac{\omega \tau+2 Q_{L, 0} \cos \psi_{0} e^{i} \psi_{0}}{(\omega \tau)^{2}+\left(2 Q_{L, 0} \cos \psi_{0}\right)^{2}}\right]\left[e^{-\left(\frac{1+i \tan \psi_{0}}{2 Q_{L, 0}}\right)_{\omega t}}-e^{-(\omega t))(\omega \tau)}\right]\right\}-\right. \\
& \left.H(t-T)\left\{1-e^{-\left(\frac{1+i \tan \psi_{0}}{2 Q_{L, 0}}\right)_{\omega(t-T)}}+(\omega \tau-i)\left[\frac{\omega \tau+2 Q_{L, 0} \cos \psi_{0} e^{i i \psi_{0}}}{(\omega \tau)^{2}+\left(2 Q_{L, 0} \cos \psi_{0}\right)^{2}}\right]\left[e^{-\left(\frac{\left.1+i \tan \psi_{0}\right)}{2 Q_{L, 0}}\right) \omega(t-T)}-e^{-\omega(t-T) /(\omega \tau)}\right]\right\}\right)
\end{aligned}
$$

and 


$$
\begin{aligned}
& \tilde{V}_{\pi}(t) /\left(V_{F 0} n_{\pi} \frac{2 \beta_{\pi}}{1+\beta_{\pi}}\right)=\cos \psi_{\pi} e^{-i \psi_{\pi}} \times \\
& \left(\left\{1-e^{-\left(\frac{1+i \tan \psi_{\pi}}{2 Q_{L, \pi}}\right) \omega t}+(\omega \tau-i)\left[\frac{\omega \tau+2 Q_{L, \pi} \cos \psi_{\pi} e^{i \psi_{\pi}}}{(\omega \tau)^{2}+\left(2 Q_{L, \pi} \cos \psi_{\pi}\right)^{2}}\right]\left[e^{-\left(\frac{1+i \tan \psi_{\pi}}{2 Q_{L, \pi}}\right) \omega t}-e^{-(\omega t) /(\omega \tau)}\right]\right\}-\right. \\
& \left.H(t-T)\left\{1-e^{-\left(\frac{1+i \tan \psi_{\pi}}{2 Q_{L, \pi}}\right) \omega(t-T)}+(\omega \tau-i)\left[\frac{\omega \tau+2 Q_{L, \pi} \cos \psi_{\pi} e^{i \psi_{\pi}}}{(\omega \tau)^{2}+\left(2 Q_{L, \pi} \cos \psi_{\pi}\right)^{2}}\right]\left[e^{-\left(\frac{1+i \tan \psi_{\pi}}{2 Q_{L, \pi}}\right) \omega(t-T)}-e^{-\omega(t-T) /(\omega \tau)}\right]\right\}\right)
\end{aligned}
$$

\section{Normalization to measured and calculated parameters}

The final step in this analysis is to connect the equivalent circuit parameters with measured quantities, or calibrations derived from numerical simulation.

\section{Measured coupling parameters}

The waveguide to cavity mode coupling can be measured by analyzing scattering parameter data. Here, we take $\beta_{\pi}=1.79$ and $\beta_{0}=0.76$. The external quality factors $\left(Q_{e x t}\right)$ are then derived from the coupling factors and the measured wall-loss quality factors $\left(Q_{w}\right)$ by $Q_{e x t}=Q_{w} / \beta$, and the fully loaded quality factor is given by $Q_{L}=Q_{w} /(1+\beta)$. The coupling to the wall, given by the wall-loss quality factor, may also be extracted from simulation (SUPERFISH, Microwave Studio, etc.)

\section{Waveguide impedance and rms power flow}

The characteristic impedance of the waveguide mode is a function of the waveguide geometry, the mode distribution and the drive frequency. We consider standard WR-284 rectangular waveguide, which transmits in single $\left(\mathrm{TE}_{10}\right)$ mode over the bandwidth needed to excite the $\mathrm{S}$-band cavities. For the $\mathrm{TE}_{10}$ mode in this geometry, the cutoff frequency is defined by geometry to be $2.08 \mathrm{GHz}$ [4.7]. The characteristic impedance for this mode is given by

$$
Z_{c}=Z_{T E}=Z_{0} \sqrt{1-\left(f_{\text {cutoff }} / f_{\text {drive }}\right)^{2}}
$$

where $Z_{0}=(\mu / \varepsilon)^{1 / 2}=376.73 \Omega$ is the free space wave impedance. The peak power flow for the forward and reverse waves in the waveguide is simply expressed by

$$
\bar{P}_{ \pm}=\frac{\left|V_{ \pm}\right|^{2}}{2 Z_{c}} .
$$

\section{Cavity shunt impedance}


As already discussed, the shunt impedance is a quantity that displays various definitions and values. Previously, our interest in analyzing the various cavity geometries was to consider the efficiency of the RF drive in providing beam power, and this resulted in one definition of the shunt impedance that folded the geometrical definition with the lowestorder effect on the longitudinal beam dynamics - the exit energy of the beam. Here, we wish to connect the fields in the drive waveguide to the fields in the cavity, and ultimately to the electric field at the cathode surface. To do this will require access to SUPERFISH (or other simulation) output files and the calibrations contained therein.

For each mode it calculates, SUPERFISH provides the geometrical shunt impedance by calculating the wall losses for a given level of mode amplitude. The line voltage is calculated along the beamline axis from the longitudinal electric field component of the mode

$$
V_{\text {line }}=\int_{\substack{\text { cavity } \\ \text { length }}} d z E_{z}(r=0, z),
$$

and the mode's shunt impedance is then

$$
R_{s}=\frac{\left|V_{\text {line }}\right|^{2}}{P_{w}}
$$

where $P_{w}$ is the power absorbed into the resistive walls.

We identify the line voltage in Eqn. (4.20) with the cavity voltage in Eqn. (4.6) or Eqn. (4.10). SUPERFISH provides a standard normalization to the field components such that the computed value of the line voltage divided by the structure length is $1 \mathrm{MV} / \mathrm{m}$.

\section{Cathode electric field}

To determine the peak electric field at the cathode surface at a given time due to a particular history of the RF drive source, we must find a calibration between the line voltage, Eqn. (4.20), and the local electric field at the cathode. The output files from SUPERFISH contain the relevant information. The electric field distribution is generated along the beamline axis (with units of $\mathrm{V} / \mathrm{m}$ ), subject the normalization condition described above. To calculate the cathode field at each time step we extract the field value from the SUPERFISH data and normalize by multiplication with the mode voltage divided by the structure length. The data from the previous SUPERFISH calculations are given in Table 4.11 below. 
Table 4.11: SUPERFISH output values for pi and zero mode quantities.

\begin{tabular}{|c|c|c|c|c|c|c|c|c|}
\hline $\begin{array}{c}\Delta \mathbf{f} \\
{[\mathbf{M H z}]}\end{array}$ & $\begin{array}{c}\mathbf{f}_{\boldsymbol{\pi}} \\
{[\mathbf{M H z}]}\end{array}$ & $\mathbf{Q}_{\text {wall, }}$ & $\begin{array}{c}\mathbf{R}_{\mathbf{s}} \\
{[\mathbf{M o h m}]}\end{array}$ & $\begin{array}{c}\mathbf{E}_{\mathbf{z}}(\mathbf{z = 0}) \\
{[\mathbf{M V} / \mathbf{m}]}\end{array}$ & $\begin{array}{c}\mathbf{f}_{\mathbf{0}} \\
{[\mathbf{M H z}]}\end{array}$ & $\mathbf{Q}_{\text {wall, } \mathbf{0}}$ & $\begin{array}{c}\mathbf{R}_{\mathbf{s}} \\
{[\mathbf{M o h m}]}\end{array}$ & $\begin{array}{c}\mathbf{E}_{\mathbf{z}}(\mathbf{z}=\mathbf{0}) \\
{[\mathbf{M V} / \mathbf{m}]}\end{array}$ \\
\hline 4.6 & 2997.9 & 12951 & 5.345 & 2.7008 & 2993.3 & 12436 & 5.810 & 3.5475 \\
\hline 7.12 & 2997.8 & 12990 & 5.234 & 2.7076 & 2990.8 & 12485 & 5.884 & 3.4985 \\
\hline 12.5 & 2997.7 & 13077 & 5.060 & 2.6806 & 2985.2 & 12574 & 6.022 & 3.4197 \\
\hline 17.6 & 2997.8 & 13510 & 5.361 & 2.6034 & 2980.7 & 12802 & 6.380 & 3.3367 \\
\hline
\end{tabular}




\section{Mode phase advance considerations}

One additional ingredient is needed to complete the analysis and have a ready tool to calculate the cathode electric field for the two resonant modes. The continuity condition, Eqn. (4.9), produces a definite phase relation between the forward and reverse voltages, and the voltages in the pi and zero modes. The relative phases of the two modes with respect to the forward voltage phase is contained within Eqns. (4.17a,b). However, this applies to the full cell plane that contains the iris connecting to the RF drive. The phase of the mode in the half-cell is shifted by the phase advance for the particular mode: $\pi$ radians for the pi mode, and 0 radians for the zero mode. This introduces an additional $\pi$ radians relative phase shift between the pi and zero modes at the cathode plane. We measure phase here as a relative phase shift at the drive frequency with respect to the phase of the forward wave, referenced to the waveguide-cavity coupling plane.

\section{RF source parameters}

We assume an ideal RF source waveform, defined in Eqn. (4.16). The rise and fall time of the waveform $(\tau)$ is assumed to be $100 \mathrm{~ns}$, for a $10 \%-90 \%$ risetime of $\sim 220 \mathrm{~ns}$. The pulse is held for $3 \mu \mathrm{sec}(T)$ before it begins to decay. The drive source central frequency is matched to that of the pi mode in the cavity. The WR-284 waveguide cutoff frequency is $2.08 \mathrm{GHz}$ for the dominant $\mathrm{TE}_{10}$ mode. The peak power of the forward wave is adjusted to produce $\sim 110 \mathrm{MV} / \mathrm{m}$ at the cathode in the pi mode.

\section{Results}

A Python script was written to generate the time dependent complex mode voltages, Eqns. (4.17a,b), from the RF power source waveform, Eqn. (4.16). For the four different frequency separation cases, we generate the corresponding waveforms for the forward and reverse waves, the peak cathode electric field in the pi and zero modes, and the combined electric field in the half and full cavity cells. This last observation corresponds to field measurements made by field probes in the two cells, and will differ significantly from the pi mode values in the presence of significant zero mode excitation. Figures 4.184.21 display these waveforms for the four cases. 
4.6 MHz separation

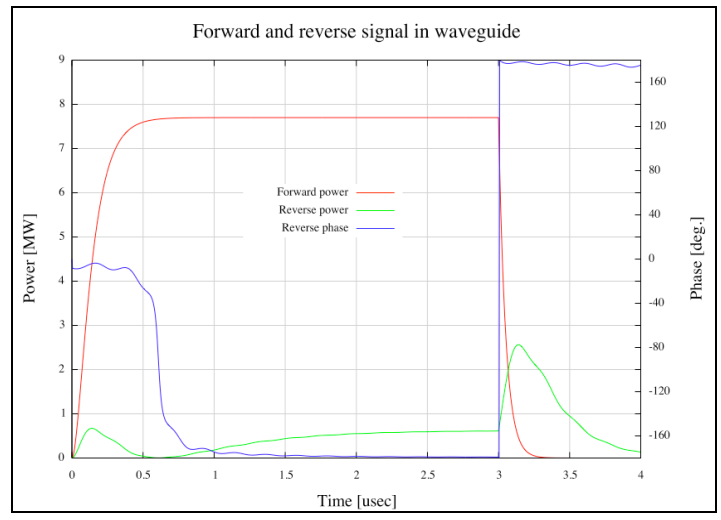

(a) Forward and reverse waveforms.

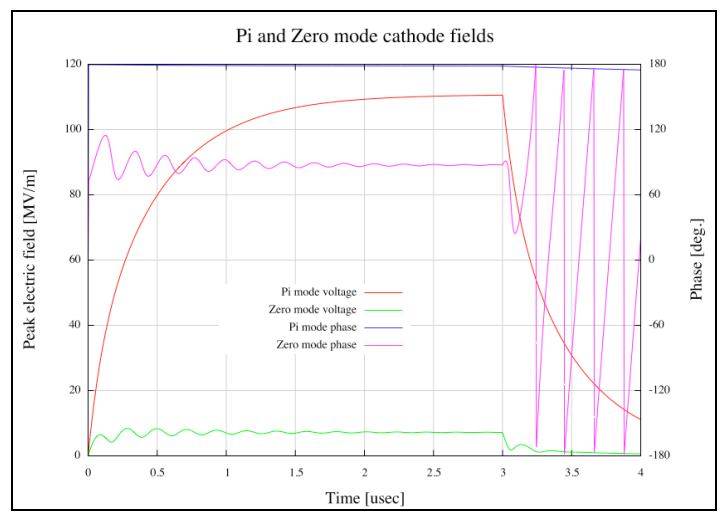

(b) $\mathrm{Pi}$ and zero mode peak fields.

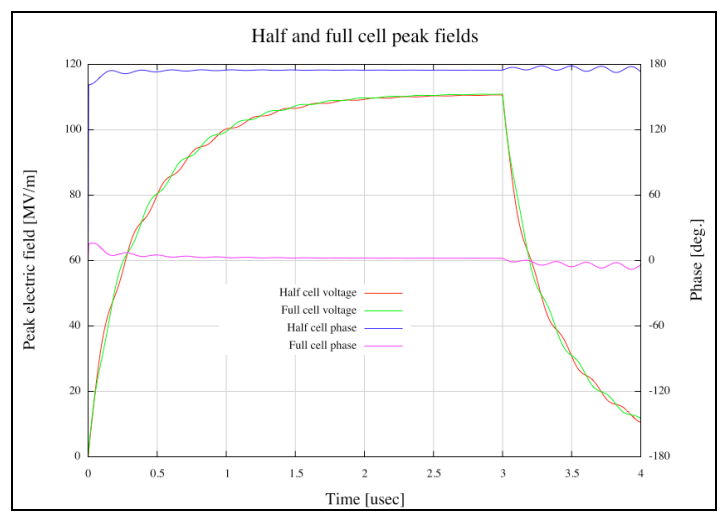

(c) Half- and full-cell peak fields.

Figure 4.18: Incident and observed waveforms for $4.6 \mathrm{MHz}$ frequency separation.
7.12 MHz separation

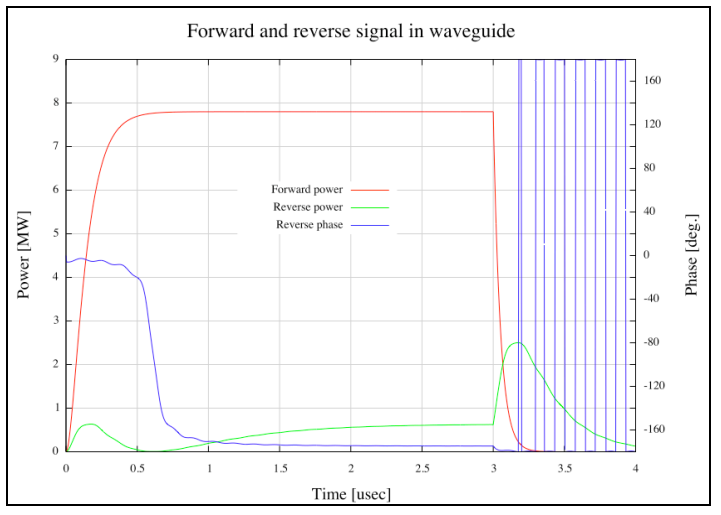

(a) Forward and reverse waveforms.

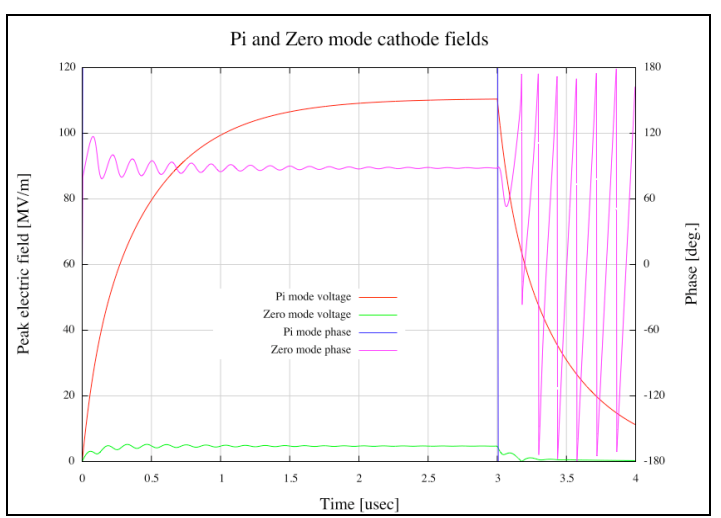

(b) Pi and zero mode peak fields.

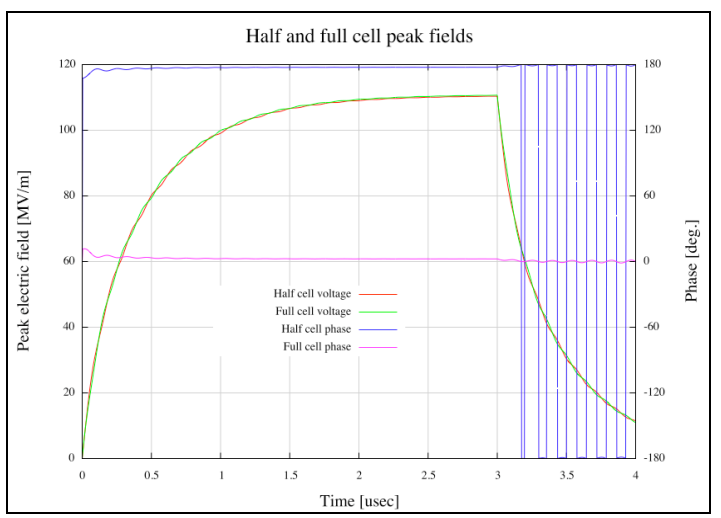

(c) Half- and full-cell peak fields.

Figure 4.19: Incident and observed waveforms for $7.12 \mathrm{MHz}$ frequency separation. 


\subsection{MHz separation}

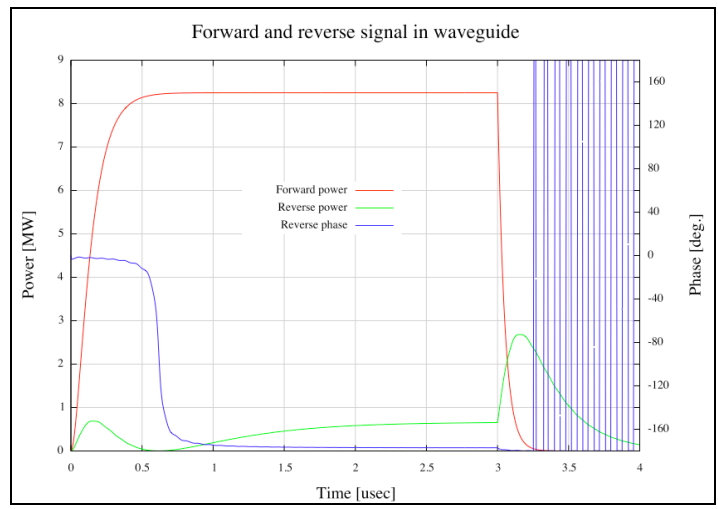

(a) Forward and reverse waveforms.

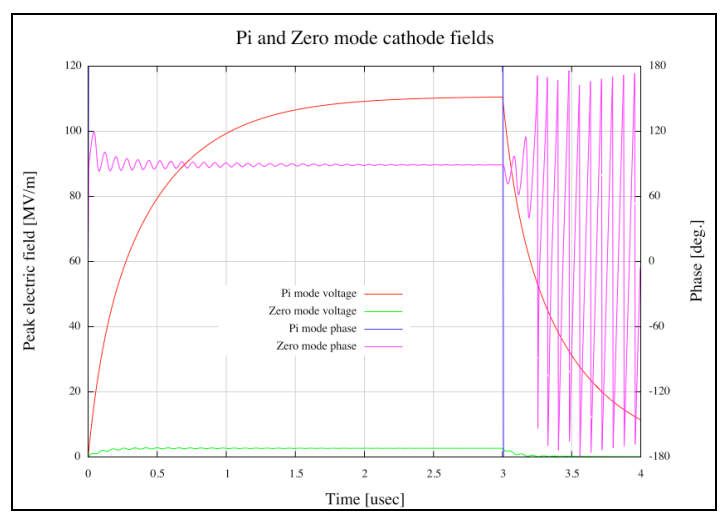

(b) Pi and zero mode cathode fields.

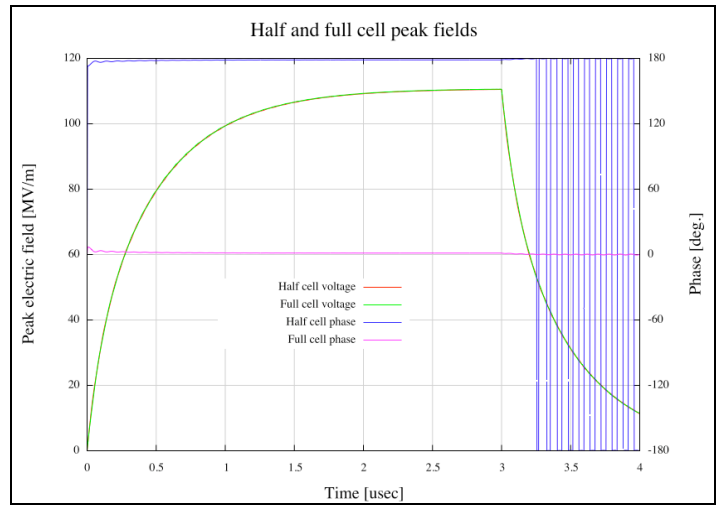

(c) Half- and full-cell peak fields.

Figure 4.20: Incident and observed waveforms for $12.5 \mathrm{MHz}$ frequency separation.

\subsection{MHz separation}

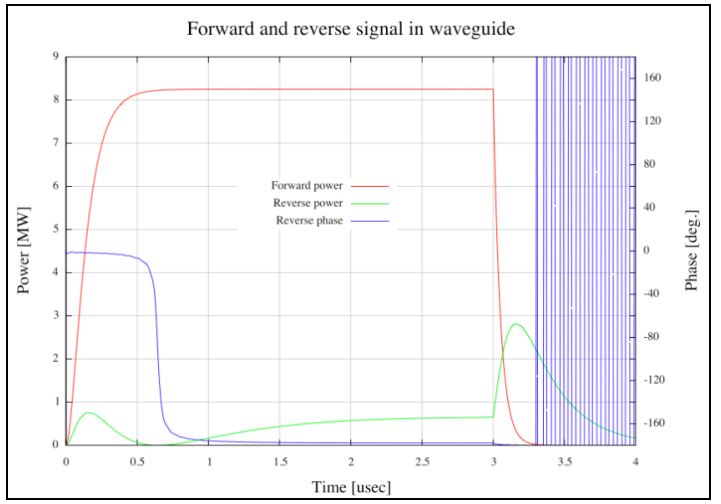

(a) Forward and reverse waveforms.

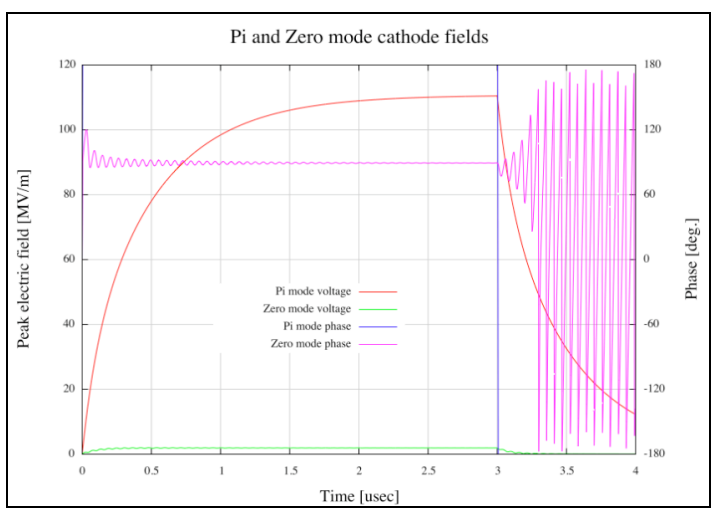

(b) Pi and zero mode cathode fields.

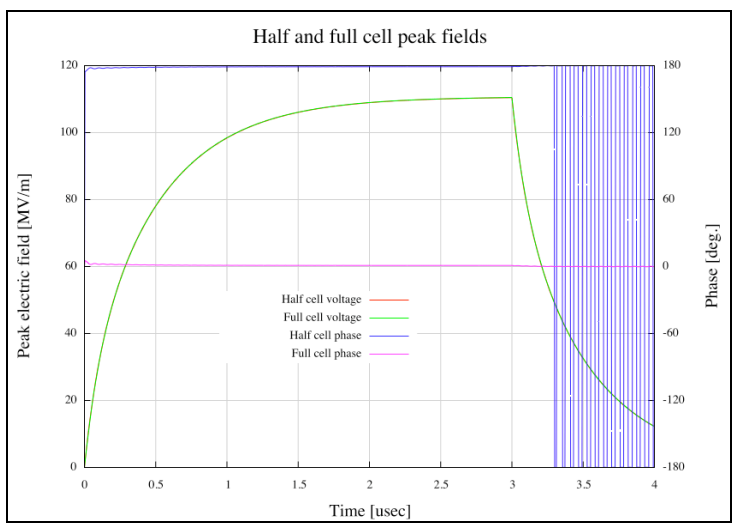

(c) Half- and full-cell peak fields.

Figure 4.21: Incident and observed waveforms for $17.6 \mathrm{MHz}$ frequency separation. 


\section{Pseudo-steady-state cathode fields and phases}

The steady state voltages may be extracted from Eqns. $(4.17 \mathrm{a}, \mathrm{b})$ by taking the limit as $t \rightarrow \infty$. The asymptotic phases of the two modes in the full cell are then given by the tuning angles, Eqn. (4.15). In pseudo-steady-state, we are examining the waveforms at the approximate time when the photoemitting laser pulse arrives towards the end of the flat-top region. For the cases presented, we perform a time-average of the voltages between $t=2.5 \mu \mathrm{sec}$ and $t=3.0 \mu \mathrm{sec}$. The time-averaged values are shown in Table 4.12. 
Table 4.12: Observed fields at steady state.

\begin{tabular}{|c|c|c|c|c|c|c|c|c|c|c|}
\hline $\begin{array}{c}\Delta \mathbf{f} \\
{[\mathbf{M H z}]}\end{array}$ & $\begin{array}{c}\mathbf{f}_{\text {drive }} \\
{[\mathbf{M H z}]}\end{array}$ & $\begin{array}{c}\mathbf{P}_{\text {forward }} \\
{[\mathbf{M W}]}\end{array}$ & $\begin{array}{c}\mathbf{E}_{\boldsymbol{\pi}} \\
{[\mathbf{M V} / \mathbf{m}]}\end{array}$ & $\begin{array}{c}\phi_{\boldsymbol{\pi}} \\
{\left[{ }^{\circ}\right]}\end{array}$ & $\begin{array}{c}\mathbf{E}_{\mathbf{0}} \\
{[\mathbf{M V} / \mathbf{m}]}\end{array}$ & $\begin{array}{c}\mathbf{\phi}_{0}^{\sim} \\
{\left[{ }^{\circ}\right]}\end{array}$ & $\begin{array}{c}\mathbf{E}_{\text {half }} \\
{[\mathbf{M V} / \mathbf{m}]}\end{array}$ & $\begin{array}{c}\phi_{\text {half }} \\
{\left[{ }^{\circ}\right]}\end{array}$ & $\begin{array}{c}\mathbf{E}_{\text {full }} \\
{[\mathbf{M V} / \mathbf{m}]}\end{array}$ & $\begin{array}{c}\phi_{\text {full }} \\
{\left[{ }^{\circ}\right]}\end{array}$ \\
\hline 4.6 & 2997.9 & 7.70 & 110.4 & 178.4 & 7.20 & 87.4 & 110.5 & 174.7 & 110.8 & 2.16 \\
\hline 7.12 & 2997.8 & 7.80 & 110.3 & 180.0 & 4.71 & 88.3 & 110.2 & 177.6 & 110.5 & 2.44 \\
\hline 12.5 & 2997.7 & 8.25 & 110.4 & 180.0 & 2.66 & 89.1 & 110.4 & 178.6 & 110.5 & 1.38 \\
\hline 17.6 & 2997.8 & 8.25 & 110.3 & 180.0 & 1.91 & 89.3 & 110.3 & 179.0 & 110.3 & 0.99 \\
\hline
\end{tabular}


This model makes several assumptions regarding the waveform of the incident, drive wave. In particular, the phase of the drive pulse is assumed to remain constant throughout the entire pulse length. Nevertheless, an analytic, closed-form expression was found from which to predict the state of the fields in the RF gun cavities at any time during the pulse, with which we may benchmark against measurements and pursue beam dynamics studies. Next, we examine the case of non-ideal drive waveforms, and the resulting behavior of the RF gun cavity fields under transient RF loading.

\subsection{Two-mode resonant cavity response under measured or simulated RF drive}

Our final approximation is to derive the behavior of the RF gun structure under nontrivial RF drive waveforms from numerical analysis of the equivalent circuit equation description. The transient and steady-state response of the cavity is determined and related to measured values of the fields in the full- and half- cells of the RF gun cavity. The GTF RF gun measurements are used to benchmark the technique presented here.

\section{Equivalent circuit equation}

We solve the circuit equation in the time domain, by integrating the differential equations from the previous note with the assumption of slowly varying envelope quantities

$$
\begin{aligned}
& \dot{\tilde{V}}_{0}+\frac{\omega_{0}}{2 Q_{L, 0}}\left(1+i \tan \psi_{0}\right) \tilde{V}_{0}=\frac{n_{0}}{Q_{\text {ext }, 0}}\left(\omega \tilde{V}_{+}+i \dot{\tilde{V}}_{+}\right), \\
& \dot{\tilde{V}}_{\pi}+\frac{\omega_{\pi}}{2 Q_{L, \pi}}\left(1+i \tan \psi_{\pi}\right) \tilde{V}_{\pi}=\frac{n_{\pi}}{Q_{\text {ext }, \pi}}\left(\omega \tilde{V}_{+}+i \dot{\tilde{V}}_{+}\right) .
\end{aligned}
$$

These equations are simplified slightly by scaling the time coordinate by the drive frequency, $\omega$. Doing so, we obtain

$$
\begin{aligned}
& \tilde{V}_{0}^{\prime}+\frac{1}{\omega \tau_{0}}\left(1+i \tan \psi_{0}\right) \tilde{V}_{0}=\frac{n_{0}}{Q_{e x t, 0}}\left(\tilde{V}_{+}+i \tilde{V}_{+}^{\prime}\right) \\
& \tilde{V}_{\pi}^{\prime}+\frac{1}{\omega \tau_{\pi}}\left(1+i \tan \psi_{\pi}\right) \tilde{V}_{\pi}=\frac{n_{\pi}}{Q_{e x t, \pi}}\left(\tilde{V}_{+}+i \tilde{V}_{+}^{\prime}\right) .
\end{aligned}
$$

In the above the cavity fill times are defined as $\tau_{0}=\frac{2 Q_{L, 0}}{\omega_{0}}$ and $\tau_{\pi}=\frac{2 Q_{L, \pi}}{\omega_{\pi}}$, and the derivatives are taken with respect to the drive phase, $\omega t$. 


\section{Input voltage waveform}

The complex voltage waveform from the RF source driver is obtained from measurements of the forward klystron pulse at a power divider in the waveguide prior to injection into the RF gun full cell. This same power divider operates as a directional coupler and also measures the reflected signal from the RF gun. The forward wave phase is measured with respect to a low-level clock signal.

The detailed time history of the voltage signal depends on details of the LLRF, amplifier chain, and delivery system to the RF gun. We have used a particular waveform from the GTF facility at SLAC as an example of a generic drive pulse [4.8]. Measurements of the forward and reverse power, the forward wave phase, and the power developed in the full cell are shown in Figure 4.22 below.

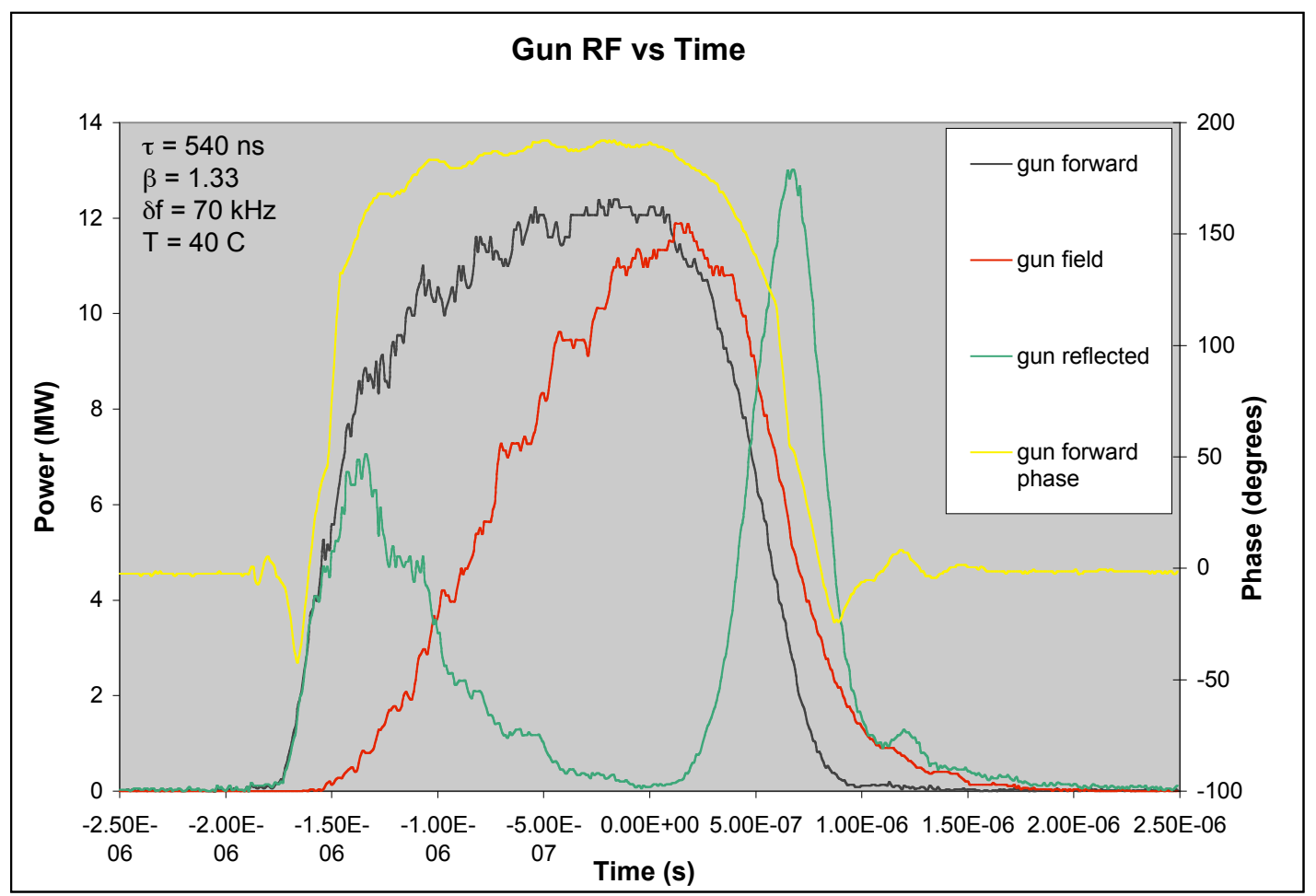

Figure 4.22: RF gun cavity waveforms from SLAC GTF measurements [4.8].

The forward wave signals shown above are then used to derive the complex voltage waveform that drives the evolution of the pi and zero mode amplitudes via Eqns. $(4.23 \mathrm{a}, \mathrm{b})$. The complex waveform is defined as

$$
\tilde{V}_{+}=\left|\tilde{V}_{+}\right| e^{-i \varphi_{+}},
$$

where the amplitude and phase are both time dependent quantities. Under the slowly varying envelope approximation (in this case with respect to the instantaneous phase of 
the drive signal), the derivative of the complex waveform is expressible in terms of the derivatives of the envelope amplitude and phase

$$
\tilde{V}_{+}^{\prime}=\left(\left|\tilde{V}_{+}\right|^{\prime}-i \varphi_{+}^{\prime}\left|\tilde{V}_{+}\right|\right) e^{-i \varphi_{+}} .
$$

\section{Results}

A Python script was written to numerically integrate the equations of motion for the time dependent complex mode voltages (Eqns. 4.23a,b) from the measured RF power source waveform (Eqns. 4.24, 4.25). The forward wave signal was analyzed by calculating a cubic spline fit, and then interpolated for particular values and time derivatives. We first analyze the GTF injector case, to benchmark the results of this method against the measured data. We then apply the method to the FERMI FEL injector, under the various cases of pi-zero mode frequency separation.

\section{GTF Case}

To solve Eqns. $(4.23 \mathrm{a}, \mathrm{b})$ requires the measurement or simulation of individual mode parameters. Several of the parameters were used as measured. Other parameters were derived from Superfish calculations, as they were not readily measurable. Finally, several parameters were varied to fit the calculated with the measured waveforms. Table 4.13 below lists the resonant mode parameters used in the calculation.

Table 4.13: GTF RF gun resonant mode parameters.

\begin{tabular}{|r|c|c|c|c|c|}
\hline Mode & $\begin{array}{c}\text { Freq. [MHz] } \\
\text { (meas.) }\end{array}$ & $\begin{array}{c}\mathbf{Q}_{\text {wall }} \\
\text { (calc.) }\end{array}$ & $\begin{array}{c}\mathbf{Q}_{\text {wall }} \\
\text { (fit) }\end{array}$ & $\begin{array}{c}\boldsymbol{\beta} \\
\text { (meas.) }\end{array}$ & $\begin{array}{c}\mathbf{R}_{\text {shunt }}[\mathbf{M W} \text { ] } \\
\text { (calc.) }\end{array}$ \\
\hline Pi & 2856.46 & 14478 & 11850 & 1.369 & 6.101 \\
\hline Zero & 2852.9 & 13701 & 12050 & 0.692 & 6.302 \\
\hline
\end{tabular}

As can be seen in Table 4.13, the resonant frequencies and coupling betas were directly measured quantities. The shunt impedances were calculated by Superfish. The wall loss quality factors were allowed to vary from their calculated values as a fit parameter. We expect a certain amount of de-Qing of the cavity wall losses from their ideal values, as calculated by Superfish. The indicated $10-20 \%$ decrease in the quality factor from the ideal value is reasonable. The remaining free parameter, then, is the actual drive RF frequency. We fit the drive frequency to a value of $2856.43 \mathrm{MHz}$. This is only detuned from the pi mode resonance by $30 \mathrm{kHz}$, well within the measurement uncertainty.

With these values, we calculate the time dependence of the mode amplitudes and phase (with respect to the forward wave phase). The fit parameter values are varied to provide a best match to the reflected power signal and the full cell field probe. The results of the fit are shown in Figure 4.23, displaying excellent agreement between the calculated and measured waveforms. The mode frequency-beating in the cavity field amplitude is 
evident. In Figure 4.24 we show the calculated electric field amplitudes at the cathode plane, as well as the phases of the pi and zero modes with respect to the forward wave phase. Figure 4.25 displays the peak electric field amplitudes and phases in the full- and half-cells of the RF gun, again displaying the mode frequency-beating behavior.

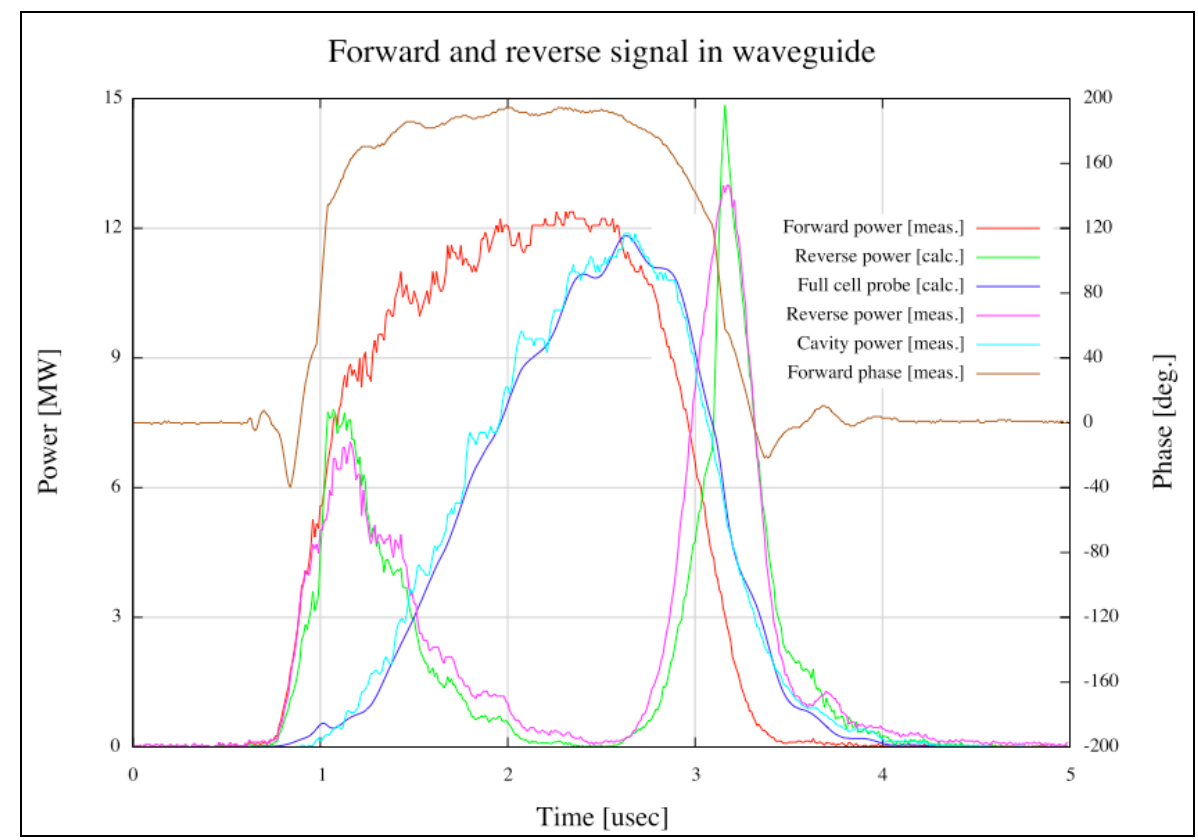

Figure 4.23: Measured and calculated time dependence of the forward and reflected power from the RF gun, and full cell cavity probe signal.

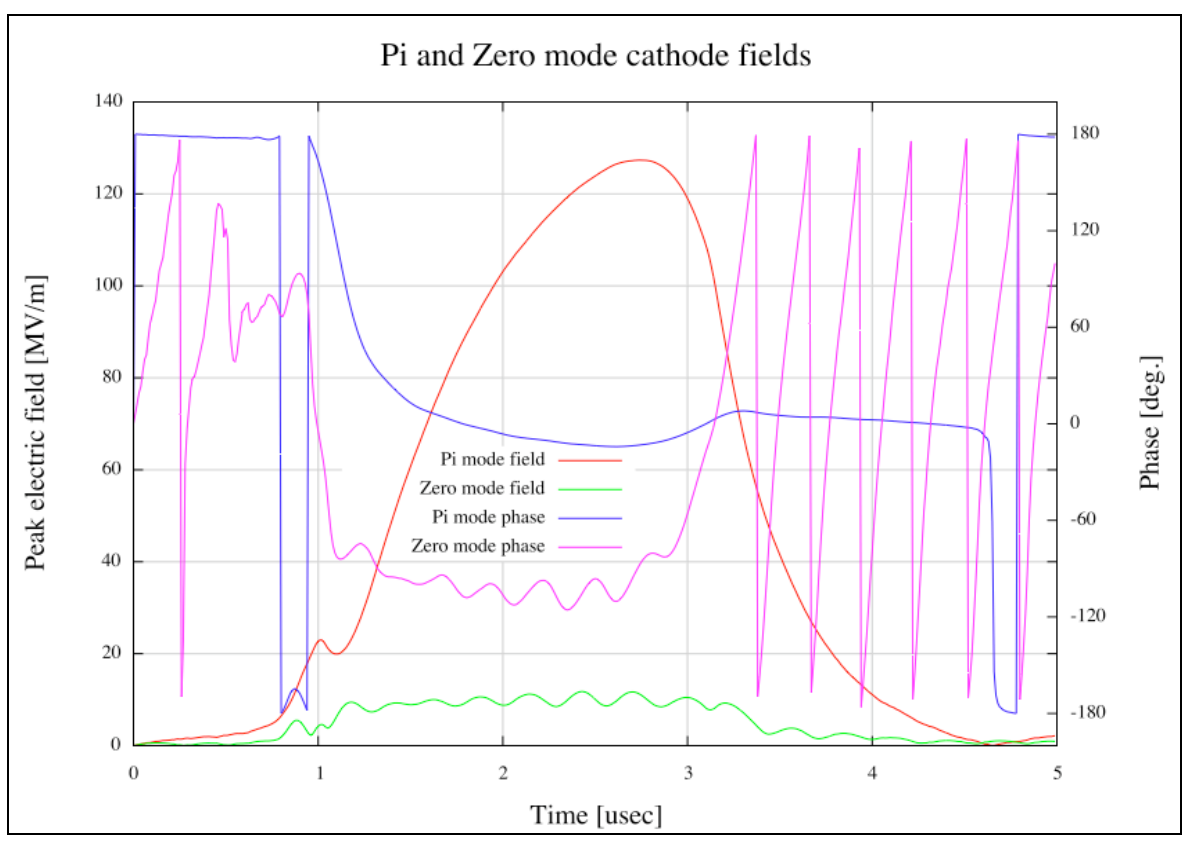

Figure 4.24: Calculated time dependence of the pi and zero mode electric field amplitudes and phases (relative to the drive RF phase) at the cathode plane. 


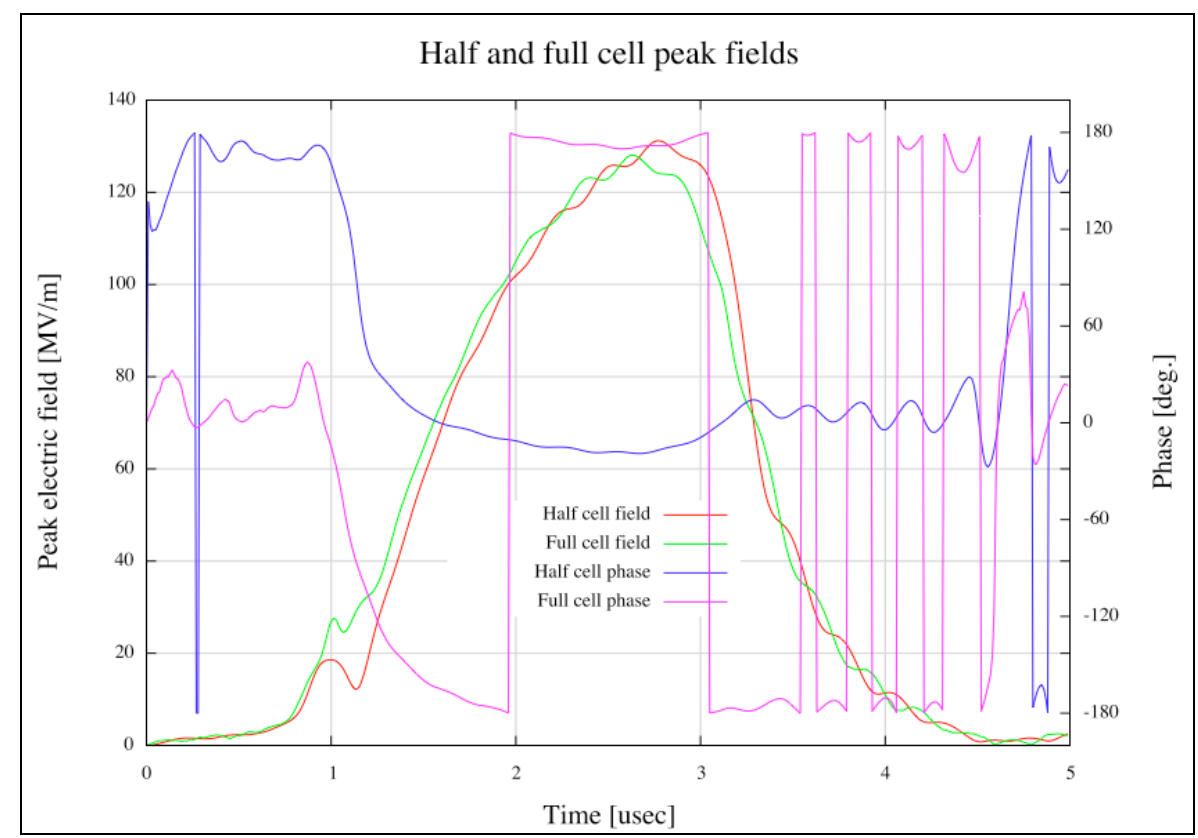

Figure 4.25: Calculated time dependence of the full-cell and half-cell peak electric field amplitudes and phases (relative to the drive RF phase).

\section{FERMI Injector Case}

For the four different frequency separation cases, we generate the corresponding waveforms for the forward and reverse waves, the peak cathode electric field in the pi and zero modes, and the combined electric field in the half and full cavity cells. This last observation corresponds to field measurements made by field probes in the two cells, and will differ significantly from the pi mode values in the presence of significant zero mode excitation.

In integrating the equations of motion, we only use parameter values calculated by Superfish, and assume that the drive frequency is matched exactly to the pi mode resonance. Additionally, we consider only near-critical coupling of the waveguide to the RF gun for both modes. In fact, we assume $\beta_{\pi}=\beta_{0}=1.05$. For all four cases the results are generically similar to the GTF results above. We empirically determine the peak forward power required to drive the peak pi mode amplitude to $110 \mathrm{MV} / \mathrm{m}$. We then locate the time along the pulse at which the peak amplitude is reached in the full-cell (this is assumed to hold our cavity voltage probe, which could just as easily be in the half-cell, or both). This is the time along the pulse at which we would launch the electron beam by firing the photocathode drive laser. The values of the peak electric fields, and their phase relative to the forward wave phase are tabulated in Table 4.14 below. 
Table 4.14: FERMI RF gun operational parameters.

\begin{tabular}{|c|c|c|c|c|}
\hline Mode separation, $\Delta \mathbf{f}[\mathrm{MHz}]$ & 4.6 & 7.12 & 12.5 & 17.6 \\
\hline$P_{\text {forward }}[M W]$ & 9.2 & 9.3 & 9.9 & 10.1 \\
\hline$t_{\text {peak }}[\mu s e c]$ & 2.82 & 2.81 & 2.81 & 2.82 \\
\hline$\phi_{\text {forward }}\left[{ }^{\circ}\right]$ & 174.3 & 175.2 & 175.2 & 174.3 \\
\hline $\mathbf{P}_{\text {cav }}[\mathrm{MW}]$ & 7.05 & 7.1 & 7.5 & 7.6 \\
\hline $\mathrm{E}_{\pi}[\mathrm{MV} / \mathrm{m}]$ & 110.3 & 109.9 & 110.2 & 110.4 \\
\hline$\Delta \phi_{\pi}\left[^{\circ}\right]$ & -6.7 & -6.8 & -6.8 & -6.6 \\
\hline $\mathrm{E}_{0}[\mathrm{MV} / \mathrm{m}]$ & 9.07 & 5.86 & 3.1 & 2.4 \\
\hline$\Delta \phi_{0}\left[{ }^{\circ}\right]$ & -92.4 & -83.6 & -83.2 & -86.1 \\
\hline$\Delta \phi_{0}-\Delta \phi_{\pi}\left[{ }^{\circ}\right]$ & -85.7 & -76.8 & -76.4 & -79.5 \\
\hline$E_{\text {half }}[M V / m]$ & 111.3 & 111.4 & 111.0 & 110.9 \\
\hline$\Delta \phi_{\text {half }}\left[{ }^{\circ}\right]$ & -11.3 & -9.8 & -8.4 & -7.8 \\
\hline $\mathrm{E}_{\text {full }}[\mathrm{MV} / \mathrm{m}]$ & 110. & 108.8 & 109.6 & 110.0 \\
\hline$\Delta \phi_{\text {full }}\left[{ }^{\circ}\right]$ & 178.1 & 176.2 & 174.8 & 174.6 \\
\hline$\Delta \phi_{\text {full }}-\Delta \phi_{\text {half }}\left[{ }^{\circ}\right]$ & 189.4 & 186.0 & 183.2 & 182.4 \\
\hline
\end{tabular}

\subsection{Beam dynamics simulations}

We have analyzed the response of the FERMI FEL RF gun to measured klystron pulse drive waveforms. The transient response of the RF cavity is described in terms of the behavior of the two lowest resonant cavity modes, and the interference they produce within the RF cavity structure. For varying RF cavity geometries, corresponding to increasing separation between the resonant frequencies of the pi and zero modes, we have generated a data set describing the optimized cavity parameters at the appropriate electron beam launching time. This analysis may be used to perform parametric sensitivity studies and to generate accurate field ratios and relative phases for beam dynamics calculations. We examine the effect of the zero-mode on a 'fiducial' solution for a regime of interest in the FERMI FEL project.

\section{Fiducial beam evolution}

We have studied the effect of zero-mode excitation on the beam dynamics in the 'medium' bunch case [4.9]. The electron beam is generated from drive laser pulse carrying a $1 \mathrm{~mm}$ (hard edge) spot size, 9ps (FWHM) pulse length with a flat-top profile and $0.5 \mathrm{ps}$ linear rise and fall times. The beam carries $800 \mathrm{pC}$ per bunch, and the initial (thermal) emittance is $\sim 0.6 \mathrm{~mm} \mathrm{mrad} \mathrm{[4.10].}$

The evolution of the transverse beam phase space is shown in Figure 4.26. The slice parameters were calculated for a $1 \mathrm{ps}$ length slice symmetrical about the bunch center-of- 
charge. Longitudinal blow-out of the bunch decrease the slice peak current from $\sim 90 \mathrm{~A}$ at generation to $\sim 75 \mathrm{~A}$ at the booster linac entrance and the injector exit.

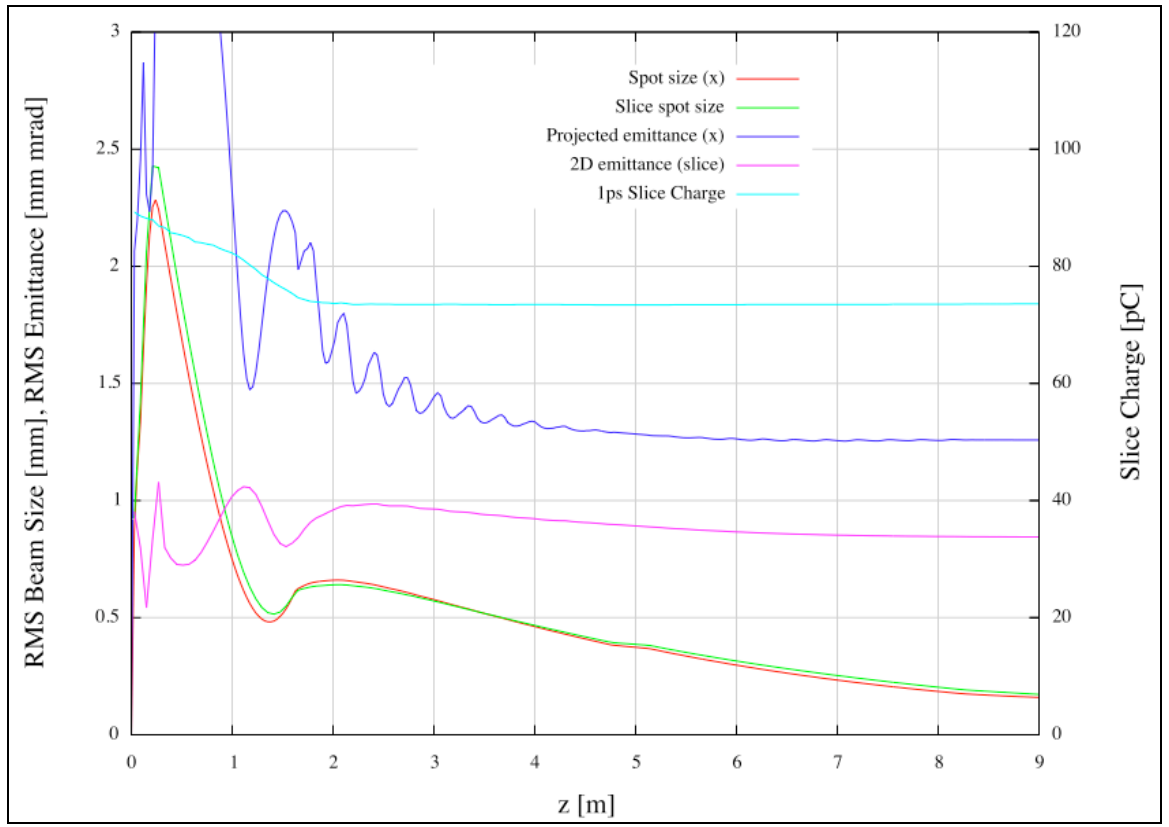

Figure 4.26: Transverse beam evolution through the injector.

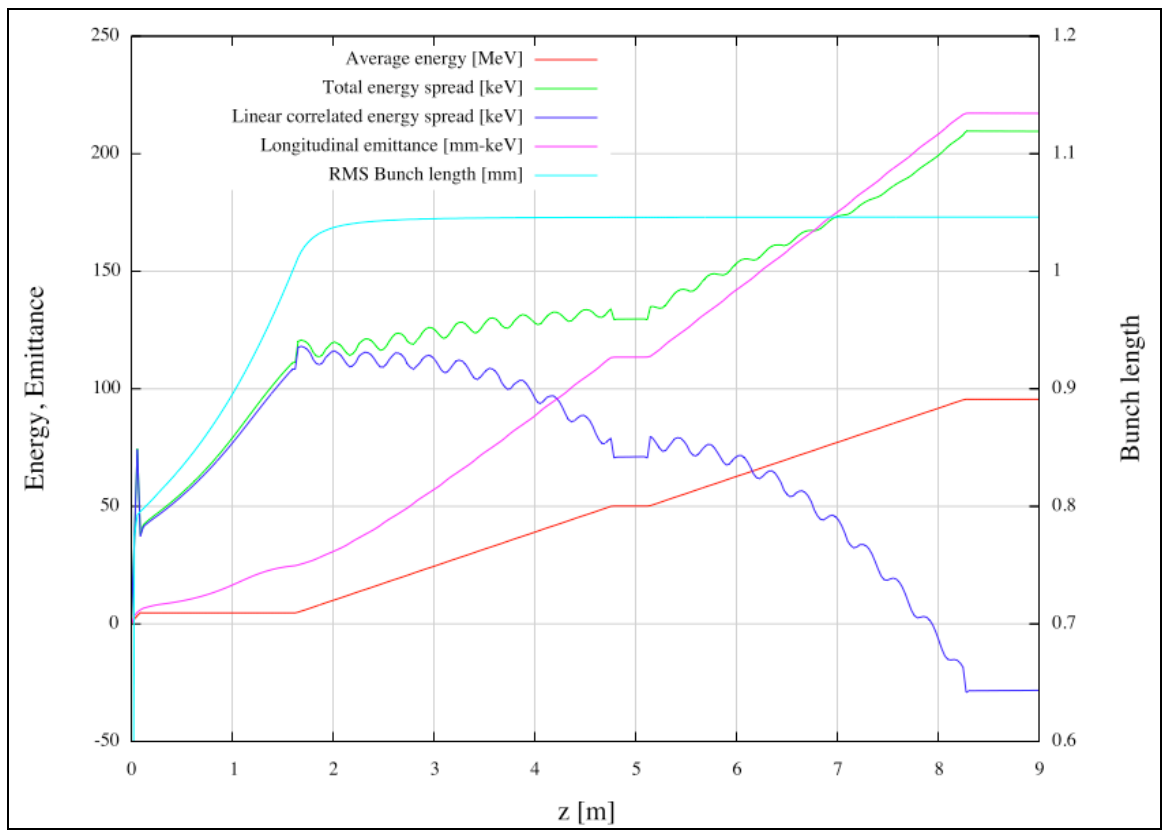

Figure 4.27: Longitudinal beam evolution through the injector.

The evolution of the longitudinal phase space is shown in Figure 4.27. The average energy of the beam is shown to increase to $\sim 95 \mathrm{MeV}$ by the time the bunch exits the injector. The RMS bunch length is shown to expand from $\sim 0.8 \mathrm{~mm}$ to $\sim 1.05 \mathrm{~mm}$, an approximately $30 \%$ increase. The region of largest expansion rate coincides with the gun- 
to-linac drift where the beam has relatively low kinetic energy and is decreasing in spot size as per the emittance compensation technique and matching to the linac optics.

The longitudinal emittance defined via

$$
\varepsilon_{s}=\sqrt{\left\langle s^{2}\right\rangle\left\langle\Delta E^{2}\right\rangle-\langle s \cdot \Delta E\rangle^{2}}
$$

where $s$ is the offset in longitudinal position, and $\Delta E$ is the offset in energy, from the fiducial particle. Figure 4.27 displays the evolution of the longitudinal emittance, total RMS energy spread, and linear correlation of the longitudinal phase space $\langle s(d \Delta E / d s)\rangle$.

The linear correlation indicates the orientation of the phase space. It has greatest meaning in the region upstream from the booster linac sections. RF field curvature in the linacs introduces quadratic (and higher order) polynomial dependencies that introduce significant distortions from linearity. The longitudinal phase space of the beam in the gun-to-linac and booster linac regions are shown in Figure 4.28.

In the gun-to-linac region, the linear correlation indicates the slope of the phase space, and hence the contribution of the correlated energy spread to the total energy spread. The total energy spread is dominated by the correlated spread contribution. The increase in the longitudinal emittance in the gun-to-linac drift is due to the increase in bunch length and the increase in nonlinear components in the phase space due to space charge forces.

We analyze the effect of the zero mode excitation on the beam dynamics. The fiducial beam dynamic above is used to generate a nominal collection of beamline parameters that determine the optimal RF gun voltages and phases, solenoid magnet peak field settings, booster linac voltages and phases, etc. For each mode frequency separation case, a set of beam dynamics simulations are produced, both with and without the presence of the zero mode, as a function of detuning the pi-mode phase with respect to the bunch launch time. 


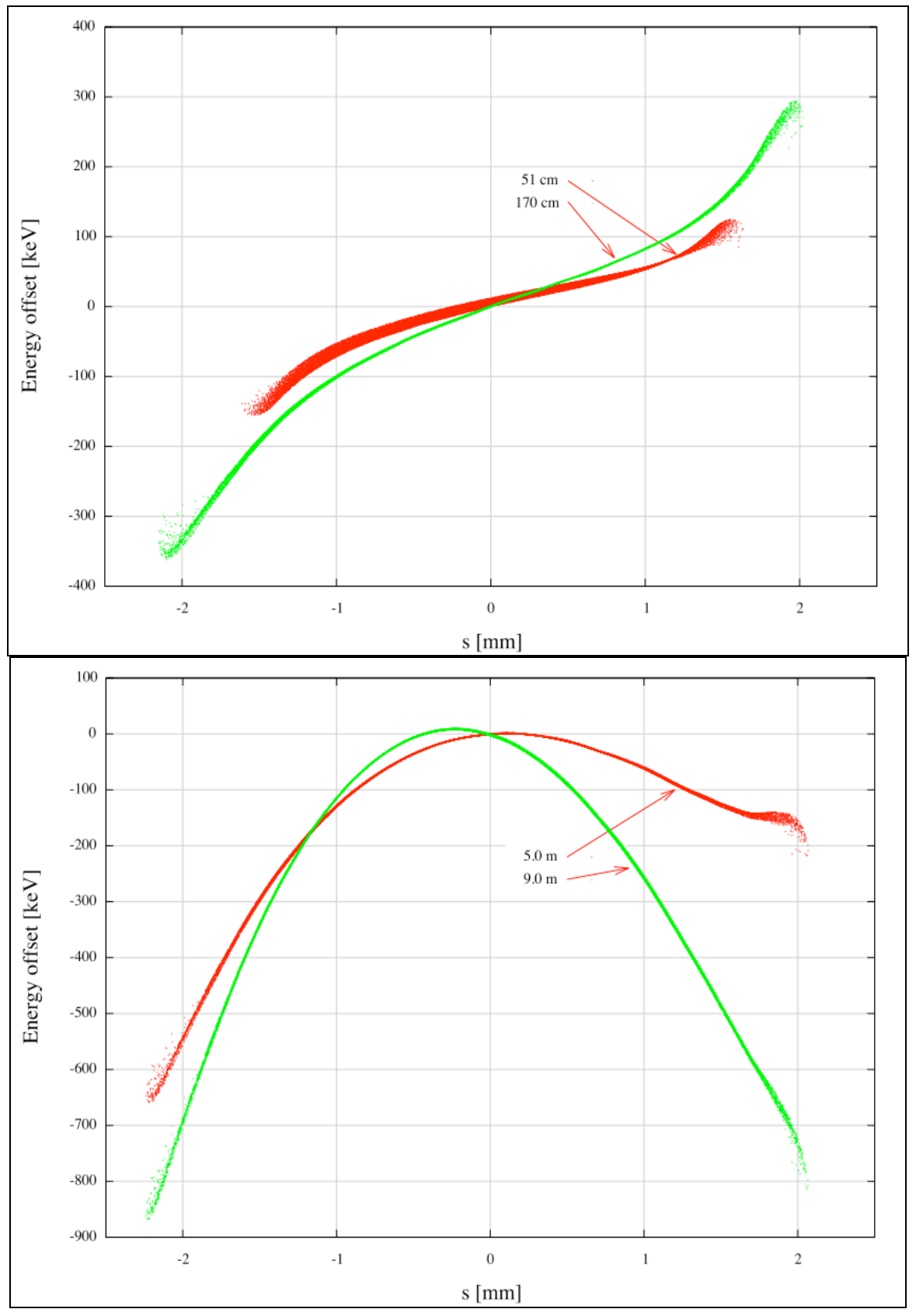

Figure 4.28: Longitudinal phase space in the gun-to-linac region $(50 \mathrm{~cm}, 170 \mathrm{~cm})$ (top), and at the exit of S0A $(5 \mathrm{~m})$ and SOB $(9 \mathrm{~m})$ (bottom).

The modes are assumed to be excited according to the GTF-like klystron pulse described previously, which introduces specific zero mode amplitudes and phases relative to the pi mode. The zero mode, when present, tracks in phase with the pi mode according to the phase difference tabulated in Table 4.14 above. This effectively produces a sensitivity study of the beam dynamics with respect to the stability of the laser arrival time at the photocathode. 
For the relatively long and intense bunches considered here, we expect that the beam dynamics will remain somewhat stable and insensitive to the zero-mode influence. Space charge forces and 'normal' RF field nonlinearities (due to the pi mode alone) will be the dominant contributors to transverse emittance and energy spread evolution. However, a quantitative understanding of the zero-mode influence is essential during commissioning and whenever bunches of short length and/or low charge are in use.

\section{Transverse phase space}

Analyzing the beam transverse phase space at the injector exit $(\sim 8.28 \mathrm{~m})$ indicates the degree to which the zero mode excitation interferes with the emittance compensation process. In general, the normalized emittance exhibits a larger degree of sensitivity to offsets in the pi-mode phase than to the presence of a perturbing zero-mode. The variation of transverse emittance with respect to the pi-mode phase is shown in Figure 4.29. The emittances calculated are average values of slice emittances, over 100 longitudinal beam slices.

As shown, small improvements in the transverse emittance occur due to changes in the cavity geometry (changes in RF transit angle of the beam) as the mode separation increases. There is also a decrease in the phase offset sensitivity at negative detuning angles. Following this, however, is an increase in the emittance sensitivity at positive phase offsets. Upon the introduction of the zero mode excitation for these cases, the transverse emittance sensitivity remains more or less constant. This is an indication that the zero mode influence is primarily confined to the longitudinal phase space.

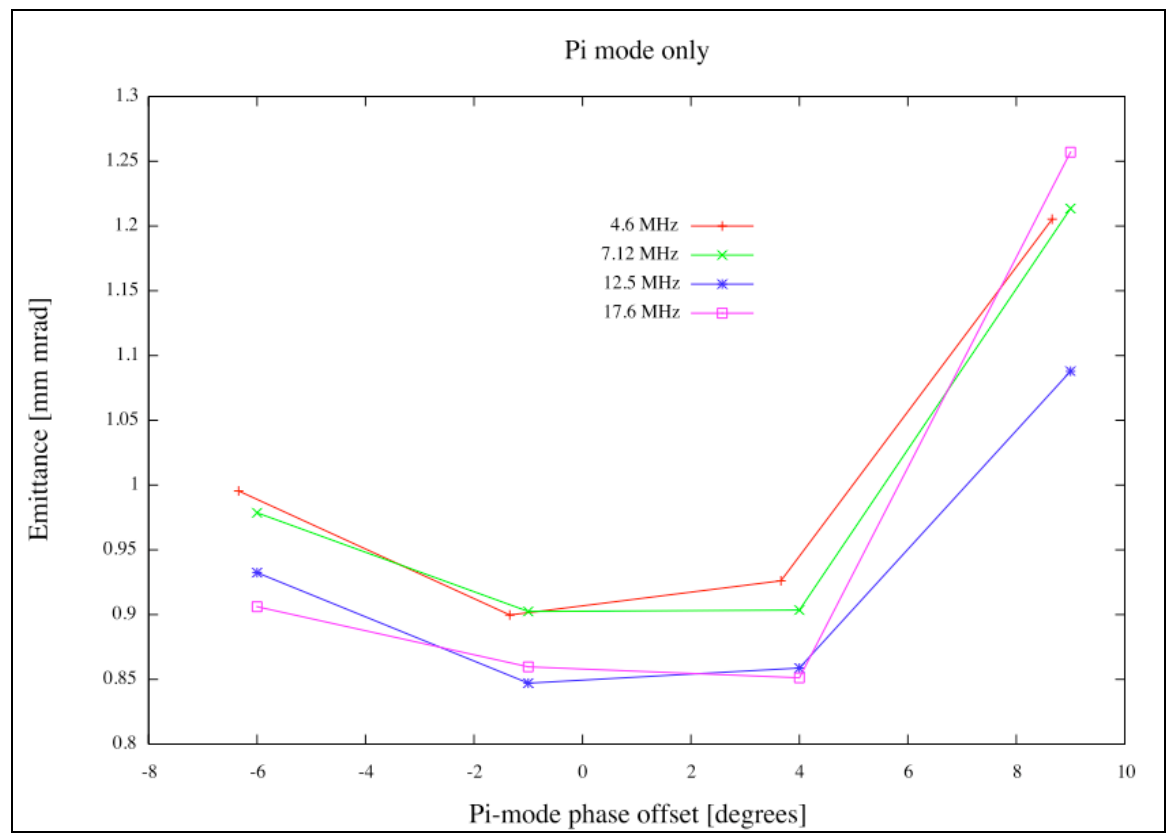




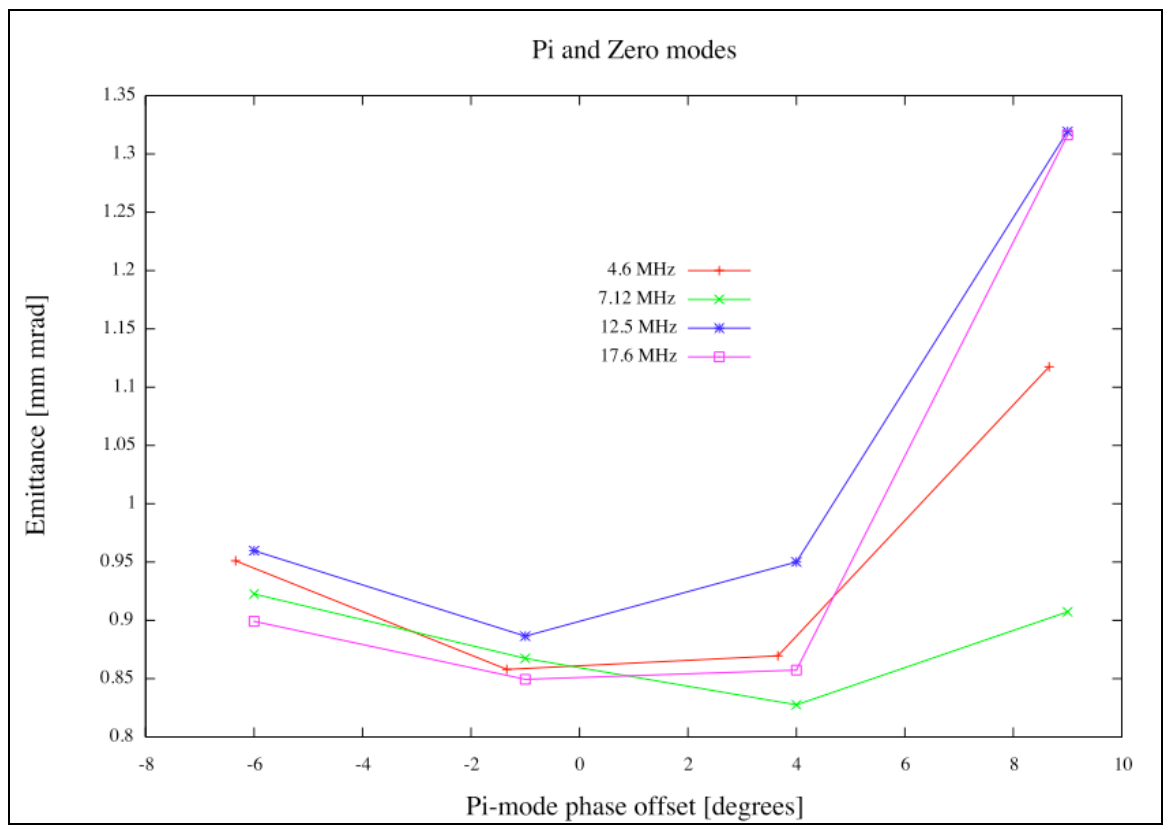

Figure 4.29: Variation of normalized transverse emittance with respect to pi-mode phase offset, for the pi-mode only cases (top) and pi+zero-mode cases (bottom).

\section{Longitudinal phase space}

The influence of the zero mode was initially noticed in studies of the correlated energy spread of photoinjector-generated electron bunches [4.11]. Beating between the two modes introduces additional correlated energy spread that must be accounted for in subsequent beam transport, measurement, and analysis.

The results of the beam dynamics runs are compared in the gun-to-linac drift region $(\sim 0.5 \mathrm{~m})$, before nonlinear RF curvature makes it difficult to extract the influence of the zero-mode. Differences are calculated in the total energy spread, linear correlation, and longitudinal emittance between the pi+zero mode cases and the pi-mode only cases, as a function of the pi-mode phase offset. The results are shown in Figures 4.30, 4.31, and 4.32. 


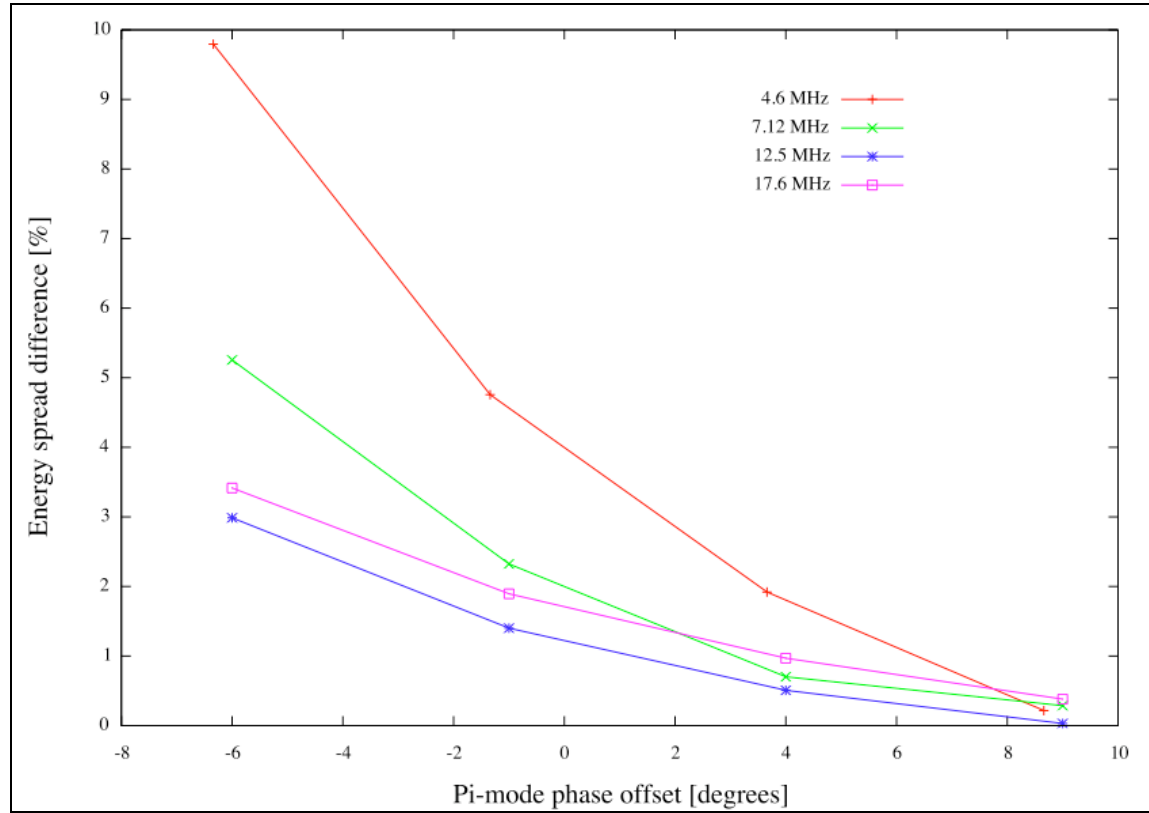

Figure 4.30: Difference in total energy spread between pi+zero mode cases and pi-mode only cases.

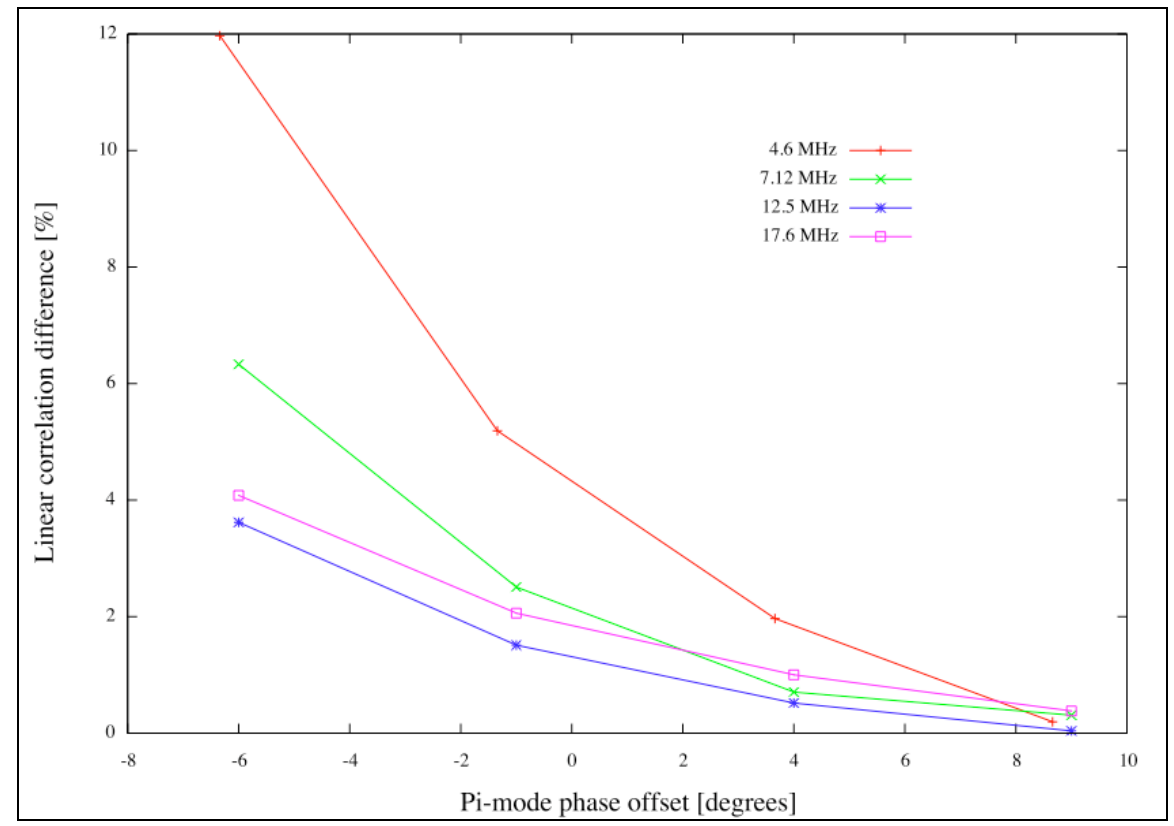

Figure 4.31: Difference in linear correlation between pi+zero mode cases and pi-mode only cases. 


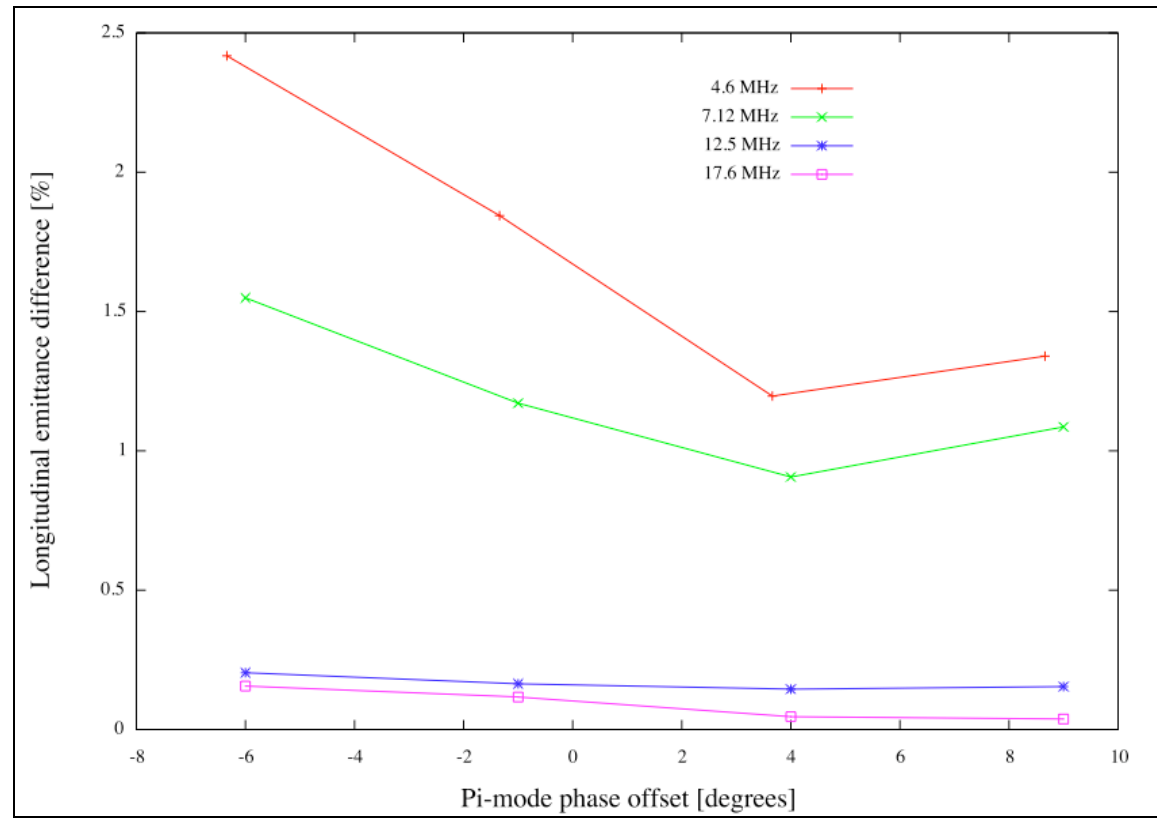

Figure 4.32: Difference in longitudinal emittance between pi+zero mode cases and pimode only cases.

Comparing the results of Figures 4.30 and 4.31, it is again evident that the correlated energy spread dominates the total energy spread in the gun-to-linac drift region in these simulations. We do observe several interesting and relevant effects of the zero-mode excitation. First, the difference in the amount of correlated energy spread generally decreases as the mode separation increases. Second, the sensitivity of the difference in the correlated energy spread with respect to the pi-mode phase offset significantly decreases as the mode separation increases. Lastly, the difference in the longitudinal emittance (Figure 4.32) is largely removed by increasing the mode separation.

\subsection{References}

[4.1] P. Craievich and V. Verzilov, "Case Study for Modification and Use of the LCLS RF Photo-Injector as an Electron Source for the ELETTRA Linac FEL”, ST/M-03/02, May 2003.

[4.2] T.P. Wrangler, RF Linear Accelerators, John Wiley \& Sons (1998).

[4.3] Microwave Studio, CST

[4.4] J. Schmerge (SLAC), private communication.

[4.5] D. H. Whittum, SLAC-PUB-7802 (April 1998).

[4.6] See, for example, M.R. Spiegel, Schaum's Mathematical Handbook of Formulas and Tables, McGraw-Hill (1968).

[4.7] Reference Data for Radio Engineers, Howard W. Sams \& Co., Inc. (1975).

[4.8] ] J. Schmerge (SLAC), private communication.

[4.9] G. Penco, et. al., "Optimization studies of the FERMI@Elettra Photoinjector", Proceedings of the 10th European Particle Accelerator Conference, Edinburgh, 2006. 
[4.10] W. Graves, et. al., Proceedings of the 2001 Particle Accelerator Conference, Chicago, 2001.

[4.10] J. Schmerge, et. al., Proceedings of the Physics and Applications of High Brightness Electron Beams, Erice, 2005. 


\section{Diagnostic Beamline Design and Layout}

The layout of the photoinjector and diagnostic beamline is presented in Figure 5.1. The low energy transport line from the RF gun to the entrance of the first booster linac (SOA) is instrumented to allow two modes of operation: direct transport ("in-line") and deflection to an energy analyzing branch beamline. Two horizontal and vertical dipole correctors (trim) and two BPMs are positioned to allow for trajectory corrections. A single magnetic quadrupole is included in the direct transport beamline to compensate for any residual phase-induced quadrupole field components in the RF gun or linac coupling cells that may interfere with emittance compensation. Standard in-line and interceptive diagnostics provide information on bunch current, transverse distribution and emittance. An energy analyzer comprised of a $90^{\circ}$ bend and three quadrupoles will be used to measure the beam energy and energy spread. Deploying a streak camera to the end of the energy analyzer beamline may allow for detailed study of the beam longitudinal phase space profile.

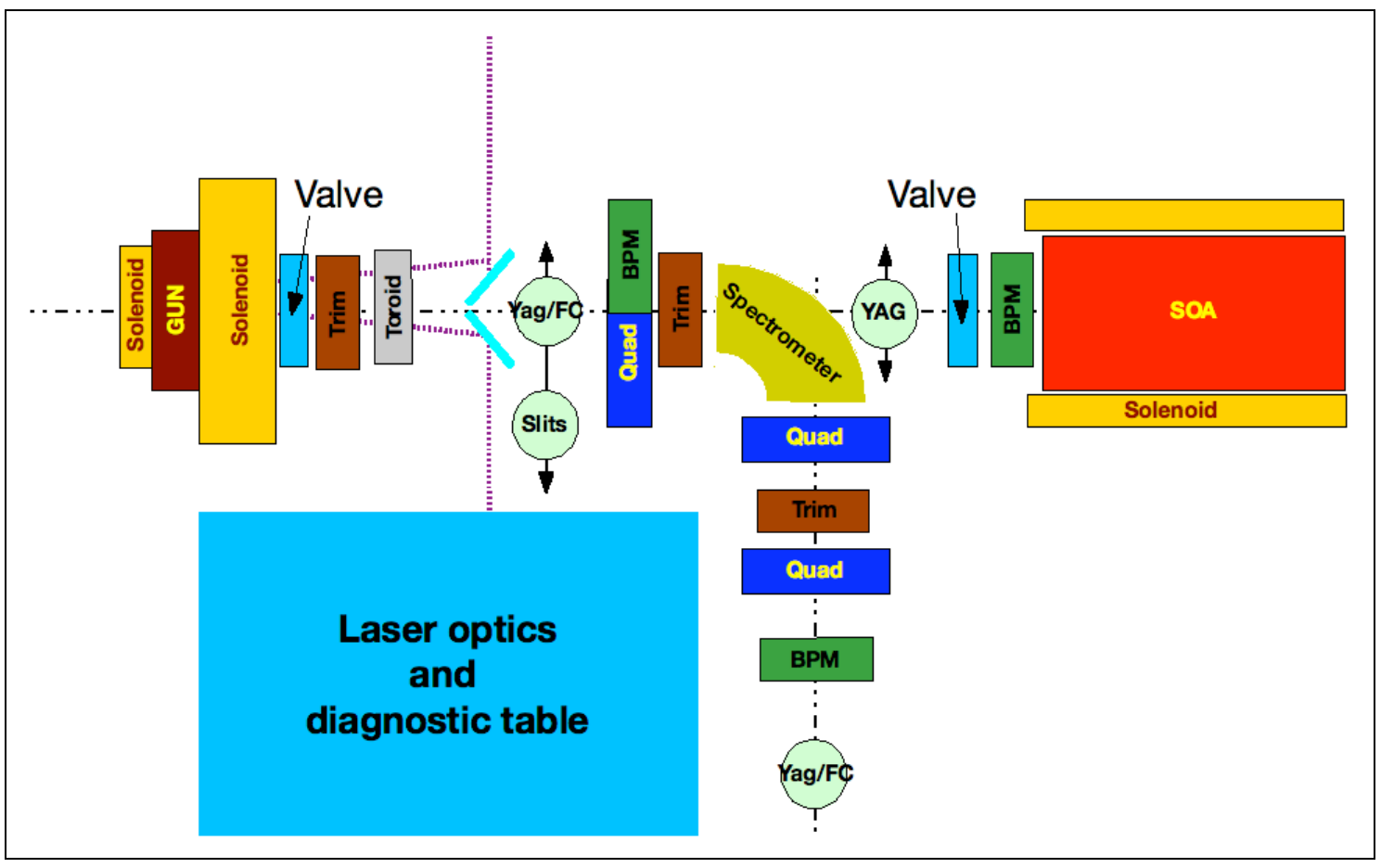

Figure 5.1: Photoinjector and energy analyzer beamline.

\subsection{RF Gun and In-line Transport Optics}

The in-line beamline is the transport line which connects the output flange of the RF gun with the entrance to the booster accelerator $(\mathrm{S} 0 \mathrm{~A} / \mathrm{S} 0 \mathrm{~B})$ and subsequent linac sections. The current physics model of the beamline assumes somewhat idealized field profiles for the beamline elements. The electron beam distribution, RF gun and linac cavity modes, solenoid magnet fields, and the resulting beam dynamics are assumed to be 
axisymmetric. In this approximation, no additional beamline elements are required to generate the high-brightness electron bunches and propagate them between the gun and booster linac.

The physics model uses a simplified, free space model of the solenoid magnets. The resulting field distribution has a fairly long longitudinal reach that may likely interfere with downstream diagnostic performance. The emittance compensating solenoid will require a detailed design to include the influence of flux clamps, while maintaining the required solenoid field integral. Along with this, a companion bucking solenoid will be designed and included to null the on-axis magnetic field at the cathode plane.

\subsection{Diagnostic Components}

\section{Beam properties to be measured directly}

The photoinjector beamline will require several types of diagnostic to perform accurate and adequate measurements on the electron beam produced by the RF gun and photoinjector laser. These measurements include: (i) bunch charge and photocathode quantum efficiency, (ii) transverse beam position, (iii) beam energy and energy spread, (iv) transverse beam profile, and (v) thermal emittance. Only the bunch charge and transverse beam position measurements may be performed with non-interceptive diagnostics. All others will require interception and/or beam deflection.

Additional diagnostic measurements will be performed on the photoinjector laser pulse, which will be described elsewhere. Of particular interest to the operation of the photoinjector will be the laser spot size and transverse distribution on the photocathode, and the total pulse energy deposited on the photocathode. These may be inferred from optical diagnostics (virtual cathode and camera, laser pulse joulemeter, etc.) located in close proximity to the RF gun. Information on the longitudinal laser pulse distribution may be inferred from analysis on the optical spectrum, or by direct measurement with a streak camera.

Beam current and bunch charge will be closely monitored during commissioning and operation to detect any slow drift in the photocathode quantum efficiency (Q.E.). Charge variations will then be communicated to the photocathode drive laser in order to correct the emitted charge by altering the laser intensity. Larger charge variations may signal large changes in the average photocathode Q.E. which will then require more extensive corrective procedures, possibly including exchange with a new or reconditioned photocathode. Producing a very small spot size at the cathode, and measuring the photo current, one may then produce a map of the photocathode Q.E. over the region of interest.

\section{Summary of standard in-line diagnostic elements}

The standard complement of diagnostics will be employed on the in-line beamline. Bunch charge will be inferred from current measurements using an inductive toroid and 
an interceptive Faraday cup (FC). The difference signals from capacitive-button-type beam position monitors (BPMs) will provide measurement of the bunch charge-centroid position, while the summation signals may also be used to measure the bunch charge after calibration. For determination of the photocathode quantum efficiency the drop-in Faraday cup will provide the most accurate measurement of the bunch charge.

YAG-coated scintillator screens, which generate optical transition radiation (OTR) when the beam passes through, will be the primary diagnostic for transverse electron beam size and distribution. Paired with a quadrupole magnet upstream, the transverse emittance of the beam may be measured determined by scanning the quadrupole magnet gradient. At low bunch charges, the thermal emittance of the photocathode may also be measured in this way.

\section{Standard diagnostic resolution and sensitivity}

The electron beam during operations will carry a charge between $0.3-1.0 \mathrm{nC}$, with a bunch length of $\sim 10 \mathrm{ps}(3.0 \mathrm{~mm})$, and a spot size diameter of several $\mathrm{mm}$. The charge measurement diagnostics should then have a nominal resolution of $\sim 10-50 \mathrm{pC}$ under normal operations. Considering the size of the beam, sufficient position resolution is $\sim 10$ $100 \mu \mathrm{m}$.

During commissioning and measurement of thermal emittances, the injector may be operated at reduced bunch charge levels, typically $\sim 50 \mathrm{pC}$ or less. For bunch charge measurement, it will then be necessary to deploy the Faraday cups on either beamline. These should maintain charge measurement resolution at less than $10 \mathrm{pC}$. The YAGcoated OTR screens have sufficient sensitivity at these lower bunch charge levels to provide gross spot size and trajectory measurements.

\section{Beam properties that may be measured with an energy analyzer and a streak camera}

A dispersive beamline that deflects the beam by $90^{\circ}$ is used to perform measurements on the average beam energy and energy spread. The optics will be described later in this note. Single-shot streak-camera measurements of the Cherenkov or OTR light radiated by the final screen may be used to probe the longitudinal beam distribution and determine the longitudinal phase space emittance.

\subsection{Beamline Layout}

The beamline is shown in Figure 5.2. The in-line Faraday cup (FC) and OTR screens (YAG) are mounted on actuators to bring them in line with the beam, and then to be retracted after measurements are performed. 
The required vacuum components are not indicated. At this time, it is assumed that a sufficient number of vacuum pumping ports will be supplied at the RF gun, at the laser window cross, and at the crosses containing the optical diagnostics and Faraday cups.

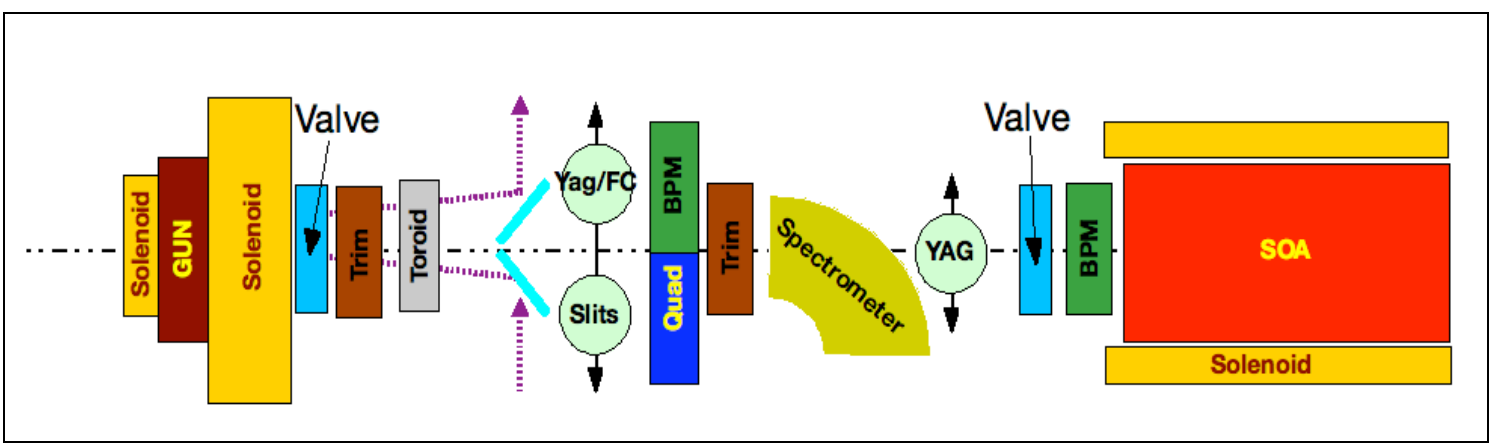

Figure 5.2: In-line beamline layout.

The diagnostic beamline layout is shown in Figure 5.3. This beamline is significantly less instrumented compared to the main, in-line beamline.

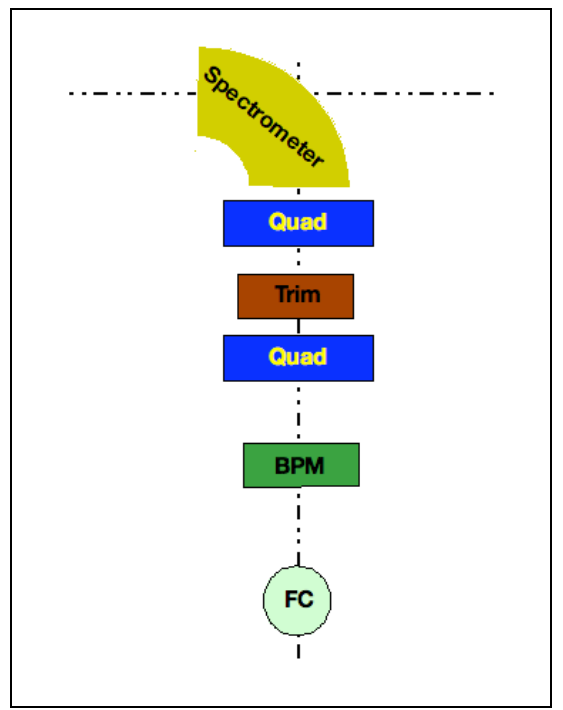

Figure 5.3: Diagnostic beamline layout.

\subsection{Energy Analyzer Optics}

To begin the modeling of the diagnostic beamline an electron bunch is generated at the cathode and transported for $89 \mathrm{~cm}$ using ASTRA. At this point, the beam is several $\mathrm{cm}$ from the entrance to the first quadrupole in the energy analyzer. The distribution phase space is analyzed both for projected and slice-averaged Twiss parameters and emittances. These parameters are then passed to a MAD model to calculate the beam transport under finite dispersion, and to optimize the locations and strengths off the beamline elements. 
The modeling and optimization of the energy analyzer beamline presented here is only a preliminary look to estimate the required field strengths and placement of the dispersive bend and quadrupoles.
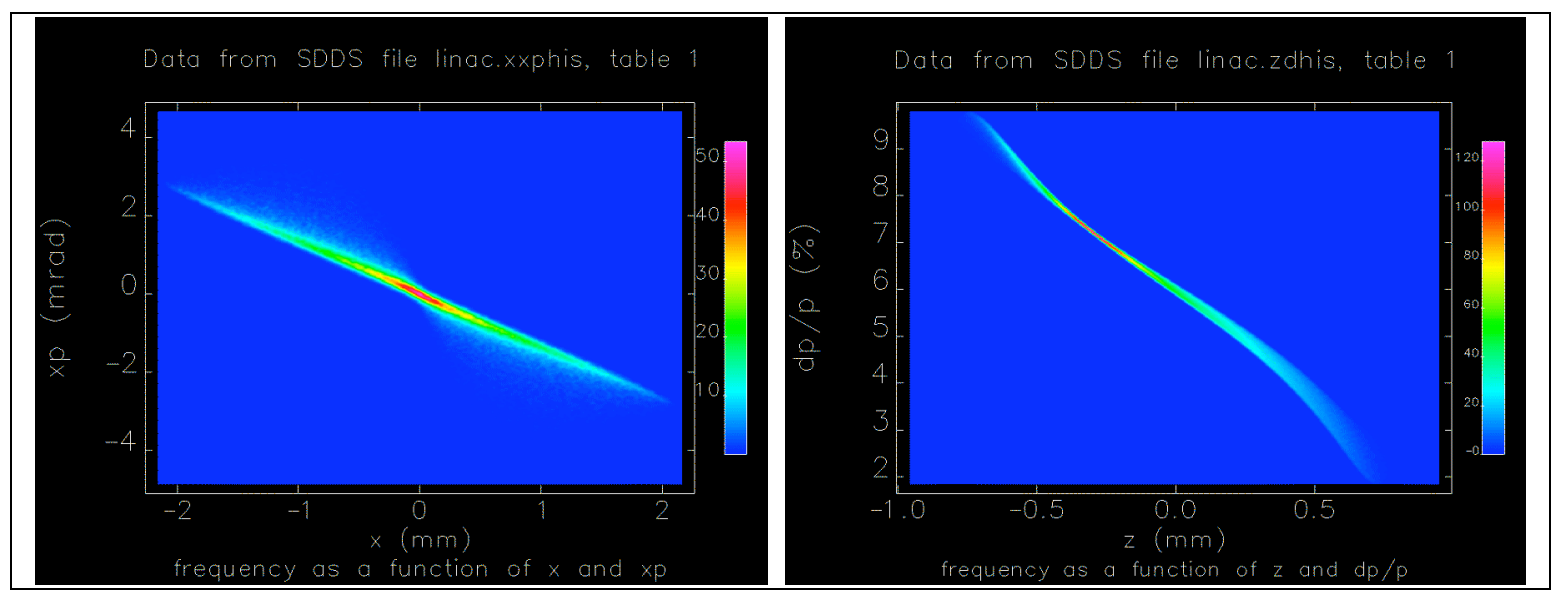

Figure 5.4: Electron beam horizontal (left) and longitudinal (right) phase spaces.

\section{Electron beam parameters}

The electron beam horizontal and longitudinal phase spaces at the entrance to the energy analyzer $(\mathrm{z}=89 \mathrm{~cm})$ are shown in Figure 5.4. The beam has been decomposed into 200 longitudinal slices, and the slice emittance and Twiss parameters are shown in Figure 5.5. The emittances and Twiss parameters for both the projected distribution and the sliceaveraged (weighted by the slice charge) beam are given in Table 5.1.

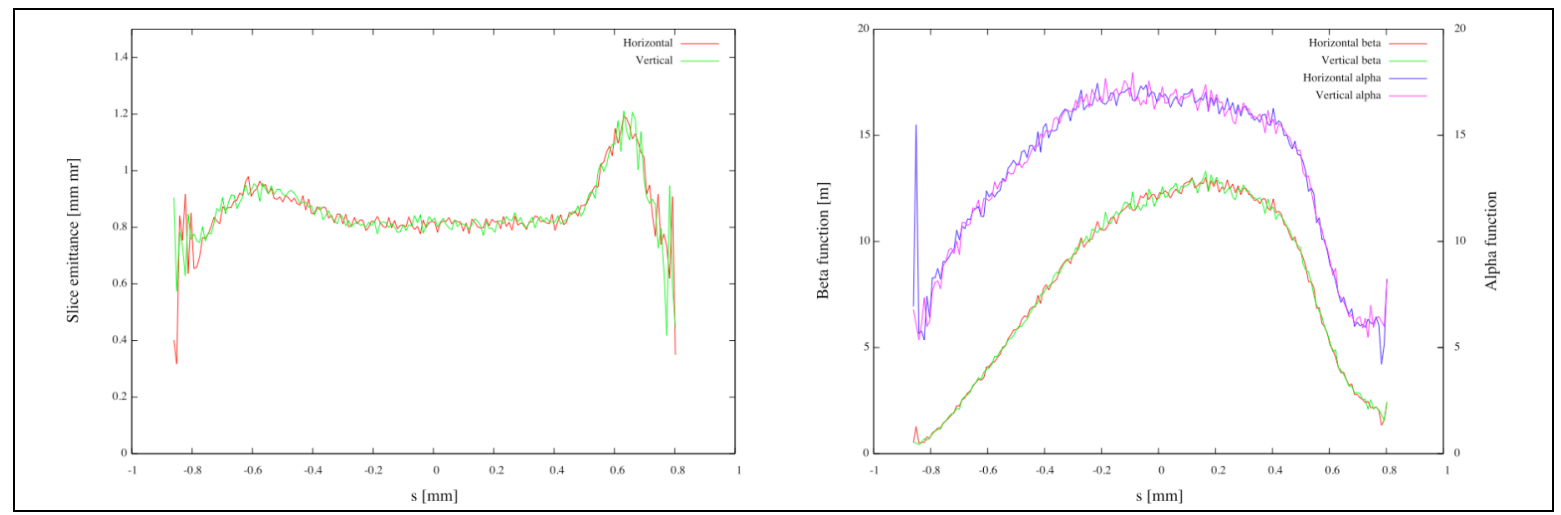

Figure 5.5: Beam slice parameters, emittance (left) and Twiss parameters (right). 
Table 5.1: Beam Twiss parameter values at spectrometer entrance ( $89 \mathrm{~cm}$ from cathode).

\begin{tabular}{|c|c|c|}
\hline Parameter & Projected & Slice-averaged \\
\hline$\varepsilon_{x}$ & 4.075 & 0.855 \\
\hline$\beta_{x}$ & 1.935 & 9.422 \\
\hline$\alpha_{x}$ & 3.097 & 14.91 \\
\hline$\varepsilon_{y}$ & 4.079 & 0.855 \\
\hline$\beta_{y}$ & 1.936 & 9.426 \\
\hline$\alpha_{y}$ & 3.094 & 14.91 \\
\hline
\end{tabular}

\section{Electron beam transport through analyzer}

The $90^{\circ}$ bend magnet forces the beam onto a $10 \mathrm{~cm}$ radius of curvature orbit. The multiple quadrupole magnets are used to generate a finite value of the dispersion function at the end of the beamline while providing for point-to-point focusing. In this way, the energy resolution of the beamline may be maximized. The beam rigidity for $5.3 \mathrm{MeV}$ electrons is $[B \rho]=0.0186 \mathrm{~T}-\mathrm{m}=18.6 \mathrm{kG}-\mathrm{cm}$.

\section{Energy Resolution at image screen}

The minimum energy resolution at the screen is determined by the optical system and the beam's longitudinal and transverse phase spaces. The dispersive beamline mixes the energy spread with emittance, and the discrimination of different particle energies is possible only if the relative energy difference is at least [5.1]

$$
\delta_{\min } \geq 2 \frac{\sigma_{x}}{D_{x}}=2 \frac{\sqrt{\varepsilon_{x} \beta_{x}}}{D_{x}}
$$

where $\delta_{\min }=\sigma_{E} / E$ is the relative energy spread; $\sigma_{x}, \beta_{x}, \varepsilon_{x}$ are the rms beam spot size, beta-function and geometric emittance; and $D_{x}$ is the horizontal dispersion in the beamline. From this model, we see that energy resolution is maximized when the dispersion function is large and the spot size is small.

The spectrometer optical functions have been designed to minimize the beam spot size, at the image screen, while maintaining a finite dispersion function value. The input deck for the MAD simulation is presented in the Appendix. The resulting optical functions are shown in Figure 5.6. 


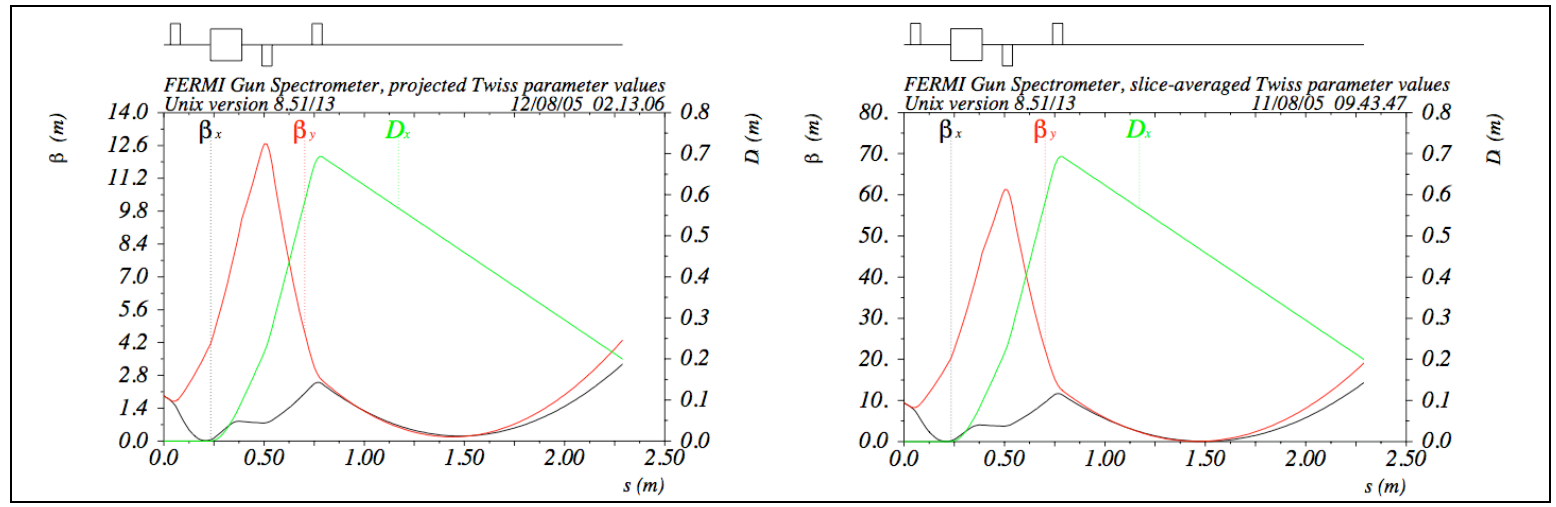

Figure 5.6: MAD models of energy analyzer showing the evolution of projected (left) and slice-averaged (right) Twiss parameters.

The screen location at $\sim 1.5 \mathrm{~m}$ coincides with the beam waist. At this point, the optical functions take the values (for a beam energy of $5.3 \mathrm{MeV}$, or $\gamma \sim 10.4$ ):

$$
\begin{aligned}
& \beta_{\mathrm{x}} \quad \sim \quad .25 \mathrm{~m} \\
& \mathrm{D}_{\mathrm{x}} \sim \quad \sim 45 \mathrm{~m} \\
& \varepsilon_{\mathrm{x}} \quad \sim \quad(1.0 \mathrm{~mm} \operatorname{mrad}) \mathrm{x}(10.4)=10.4 \mathrm{~mm} \mathrm{mrad}
\end{aligned}
$$

The minimum relative energy resolution is then $\delta_{\min } \geq 0.23$, or $\sim 1.2 \mathrm{MeV}$.

The magnet strengths from the MAD model are listed in Table 5.2.

Table 5.2: Magnets for energy analyzer

\begin{tabular}{|r|c|c|}
\hline Magnet & $\begin{array}{c}\left.\text { Bend angle } \mathbf{(}^{\circ}\right) \text { or } \\
\text { Focusing strength, } \mathbf{k}\left(\mathbf{m}^{-\mathbf{2}}\right)\end{array}$ & $\begin{array}{c}\text { Field } \\
\text { Strength }\end{array}$ \\
\hline Bend & $90^{\circ}$ & $1.9 \mathrm{kG}$ \\
\hline Q1 & 80 & $1.5 \mathrm{~T} / \mathrm{m}$ \\
\hline Q2 & -60 & $1.1 \mathrm{~T} / \mathrm{m}$ \\
\hline Q3 & 65 & $1.2 \mathrm{~T} / \mathrm{m}$ \\
\hline
\end{tabular}

The beamline presented here represents an initial compromise between the requirements of producing a high brightness electron beam, which tends to require shorter drift lengths between the RF gun and the first booster linac for proper emittance compensation, and the needs of the diagnostics and other instrumentation required to diagnose and to preserve the high quality of the beam. 


\section{Appendix: MAD Input Deck}

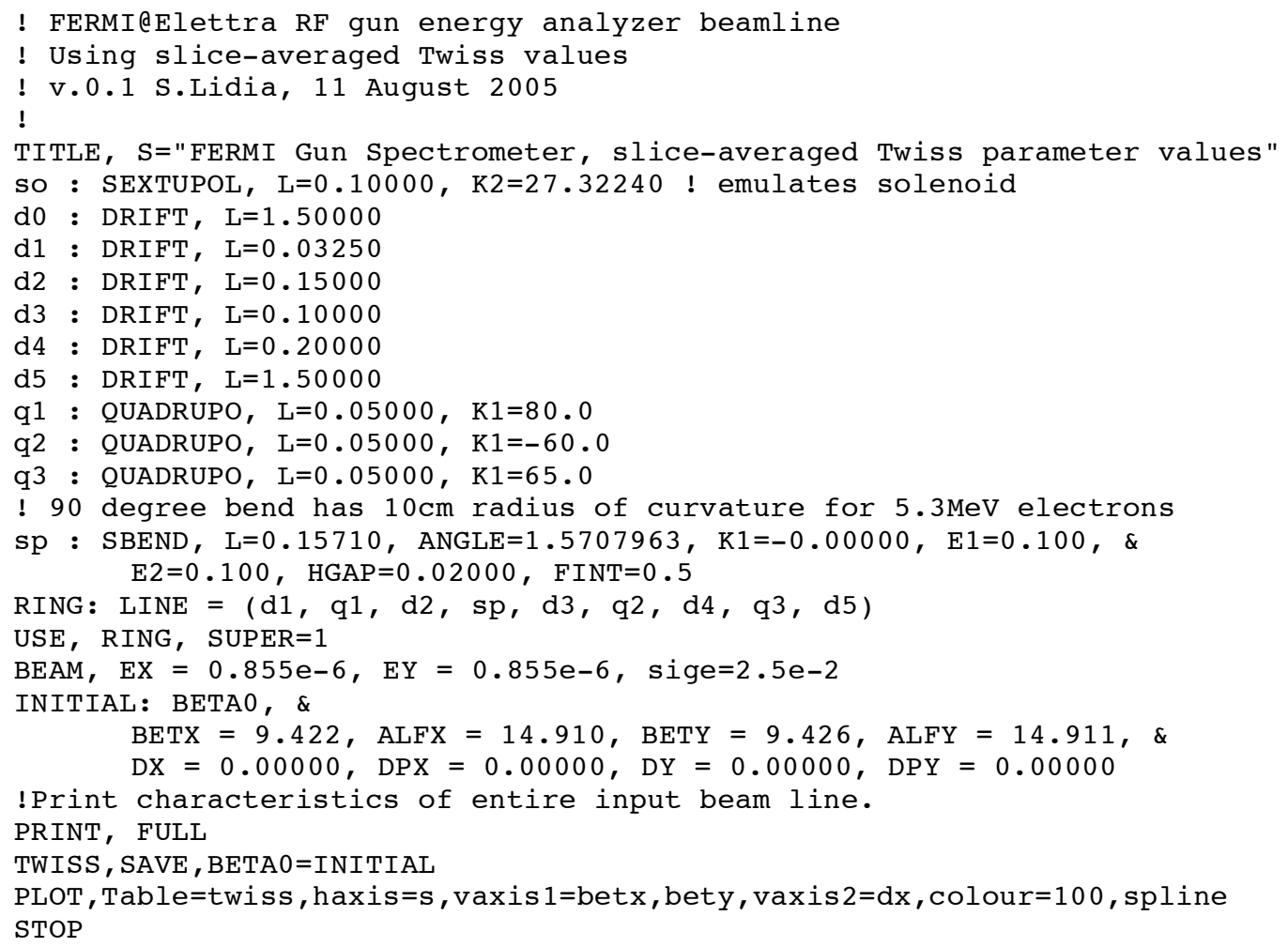

\subsection{References}

[5.1] H. Wiedemann, Particle Accelerator Physics, Vol. I, Springer, 1999. 


\section{Injector Performance Studies}

The beam dynamics in the injector system has been modeled extensively from emission at the photocathode to the exit of the booster accelerator modules using 2D and 3D spacecharge tracking codes. There have been certain approximations taken regarding the beamline and its components. First, the entire beamline has been assumed to possess perfect azimuthal symmetry about the beamline axis. Second, the model of the RF gun in these simulations has only assumed the presence of the $\mathrm{TM}_{010-\pi}$ ('pi') mode. We have deferred consideration of the details of optimization until the actual RF gun design has been finalized, and the pulse characteristics of the klystron modulator and RF drive are measured and known. As shown in the previous sections, the lower-frequency, 'zero' mode response is a complicated function of the RF pulse history and gun geometry. The optimization studies presented here are then to be considered for their generic worth, and will require some small recalibration once the RF gun behavior has been more completely characterized. Lastly, longitudinal wakefield effects from the booster accelerator structures have been included in an approximate form. Time-dependent longitudinal impulses are calculated based on the published wakefield data and the longitudinal distribution of the bunch charge. These impulses are then applied only at the exit of each section, and the beam is then allowed to continue to propagate.

Flexibility in the beamline design and tuning for optimum performance has required some compromise. Early studies of emittance compensation required the entrance of the first booster accelerator to be placed at $\sim 1.4 \mathrm{~m}$ from the photocathode. Space requirements for the beamline instrumentation, and inclusion of the diagnostic beamline, have increased the minimum drift distance between the RF gun exit and the first booster structure entrance. The result is a re-optimization of the beam dynamics assuming a cathode-booster separation of $\sim 1.6 \mathrm{~m}$.

This section discusses the three bunch classes so far considered: 'short', 'medium', and 'long'. The 'short' bunch case design is presented for completeness only. At this time, the 'medium' and 'long' bunch cases are define the baseline configurations. However, the 'short' bunch case may retain some utility during commissioning, or in some special cases.

For the 'medium' and 'long' bunch cases, we consider two types of laser excitation at the photocathode: (i) flat-top generation, ad (ii) ramped current generation. The former produces a well-characterized beam distribution that has been optimized in previous work, and represents a baseline metric for performance. The latter case was introduced after considerations of performance optimization in the remainder of the FERMI FEL linac. In particular, accelerating structure and chicane CSR wakefield effects have driven the electron beam design to include a quasi-linear head-tail ramp in the instantaneous current to produce a 'flat-flat' beam (i.e. uniform in current and energy) profile at the entrance of the FEL undulators. This section describes the optimized beam dynamics for the various bunch cases. Following that are parametric sensitivity studies and parameter jitter analysis. 


\subsection{Optimum Baseline Configurations}

\section{Short Bunch}

We have performed simulations by GPT and ASTRA in the 'short' bunch regime by varying the charge extracted from the cathode (around 400pC) and the bunch length (around 5ps) in order to produce a bunch at the exit of the photoinjector consistent with the requirements coming from the linac optimization [6.1]. Taking into account the emittance growth in the linac and bunch compressors, emittance values targeted for the injector are obviously more restrictive than required at the entrance of the undulator. Accordingly, the goal on the normalized emittance values at the end of the photoinjector is less than $1.5 \mathrm{~mm}$ mrad for the projected emittance and less than $1.0 \mathrm{~mm}$ mrad for the slice emittance, which should exhibit a flat-like behavior along the bunch, at least concerning the central core [6.2]. In addition, we have found that the longitudinal bunch profile at the exit of the photoinjector represents a very strong constraint from the point of view of the transport through the linac. A variation in the bunch length greater than $\sim 10 \%$ leads to a "mismatching" with the bunch compressor parameters setting [6.3]. Following the linac requirements we have fixed the charge per pulse extracted from the cathode to $330 \mathrm{pC}$ and have optimized the bunch length at the exit of SOB section to about 5.5ps (FWHM). We have studied several longitudinal laser pulse widths in order to obtain the desired bunch length at the entrance of the linac. We have considered a laser spot size of $1 \mathrm{~mm}$ radius and uniformly distributed transversely, so that the electron bunch has a consequent thermal emittance of about $0.6 \mathrm{~mm} \mathrm{mrad}$, as given by the formula $\varepsilon_{t h}(\mathrm{~mm} \mathrm{mrad})=0.16+0.93 \cdot \sigma_{X}(\mathrm{~mm})[6.4]$.

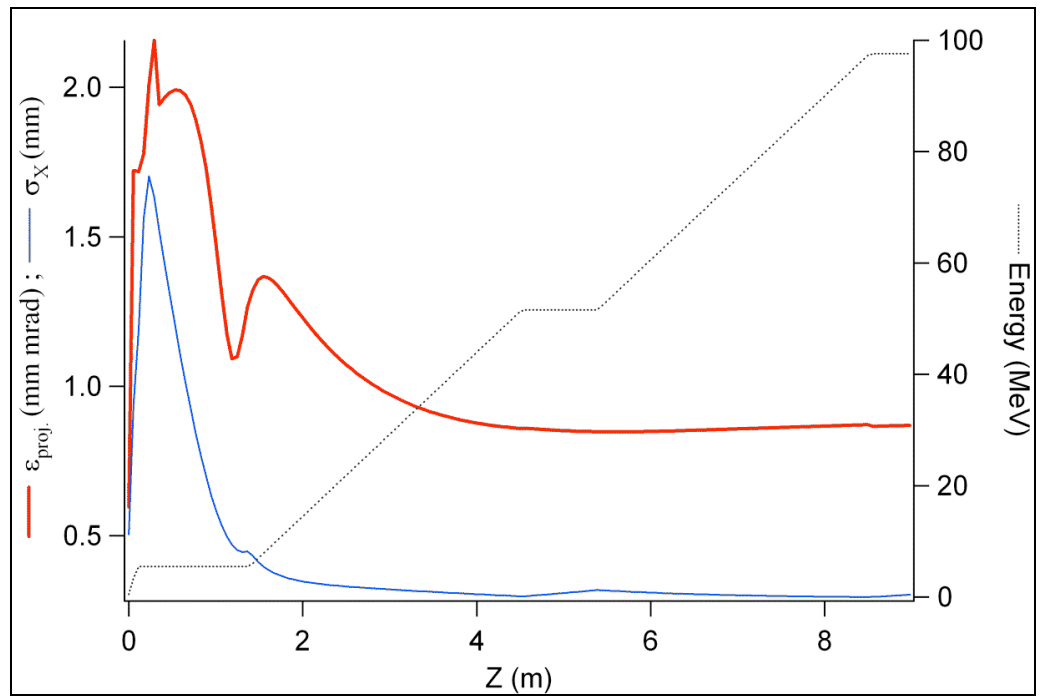

Figure 6.1: Projected radial emittance, radial spot dimension and energy along the photoinjector beamline for the short bunch regime; SOA at $1.4 \mathrm{~m}$ from the cathode.

Following the classical technique of emittance compensation [6.5], we have optimized the RF gun injection phase, obtaining $24 \mathrm{deg}$ (with respect to the RF zero crossing), and the strength of the compensation solenoid, obtaining 2450 Gauss. A conservative value of 
$110 \mathrm{MV} / \mathrm{m}$ for the gun accelerating gradient has been considered, even if better performances can be obtained by increasing it to $140 \mathrm{MV} / \mathrm{m}$.

In this condition the matching point, where the SOA section has to be installed, was found to be at a distance of $1.4 \mathrm{~m}$ from the cathode surface. As foreseen the low accelerating gradient of SOA is not sufficient to compensate the emittance completely, so it is necessary to add a focusing element - a long solenoid around the SOA section, which is set to 700 Gauss. Figure 6.1 shows the projected emittance behavior, the RMS radial dimension of the bunch and the bunch energy along the photoinjector beamline. At the end of SOB the projected radial emittance attains the value of $0.84 \mathrm{~mm}$ mrad. This found solution fits the linac requirements concerning the longitudinal bunch profile consists in starting with a laser profile as in Figure $6.2(\mathrm{FWHM}=4.5 \mathrm{ps}$ and rise $/$ fall time $=0.5 \mathrm{ps})$.

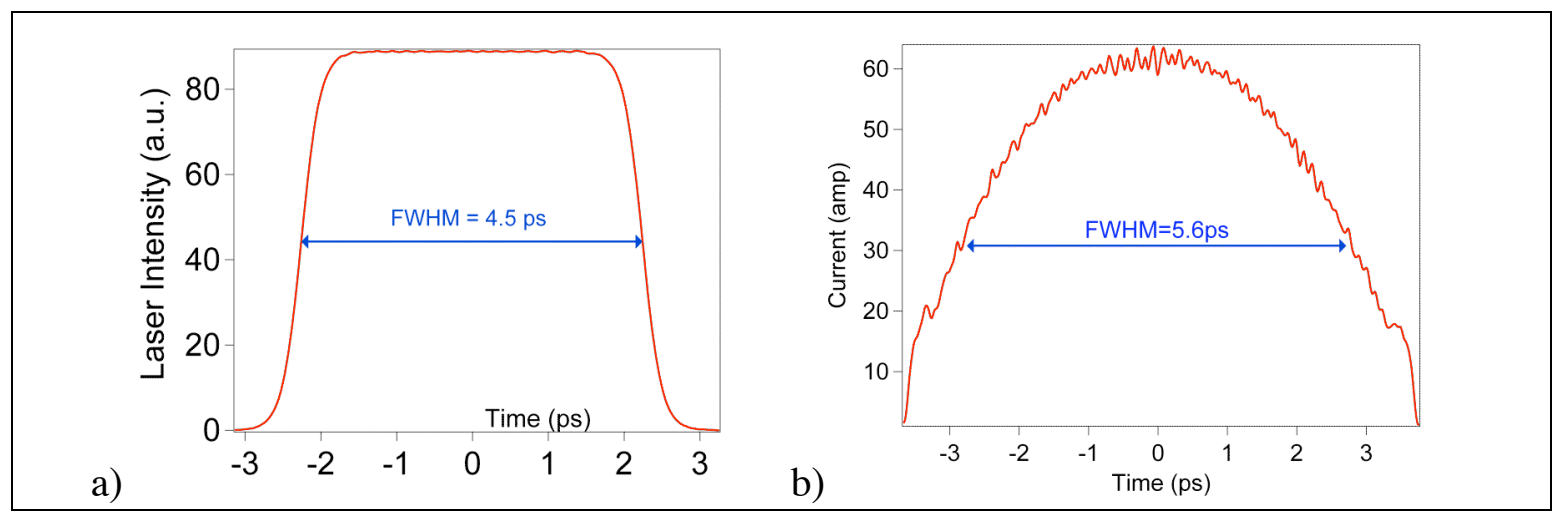

Figure 6.2: a) Temporal laser profile; b) Longitudinal bunch profile at the exit of SOB.

The electron bunch originated from that laser profile is stretched during the propagation in the photoinjector by the space charge effect, and at the exit of SOB the bunch profile attains to 5.6ps (FWHM) (Figure 6.2(b)), with a peak current greater than 60 A. All simulation results shown in Figures 6.1 and 6.2 refer to GPT tracking with 200k particles and good agreement with ASTRA has been verified. This number of particles is acceptable not only from the point of view of the optimization of the emittance compensation procedure, but even for the slice analysis. We divide the longitudinal bunch profile at the end of SOB into 100 slices and calculate the emittance and the energy spread of each slice, obtaining the plot reported in Figure 6.3.

The slice emittance is kept quite constant around $0.65-0.70 \mathrm{~mm}$ mrad along a large part of the bunch, while the slice energy spread, excluding the head/tail spikes, varies from a minimum of $2 \mathrm{keV}$ to a maximum of $4 \mathrm{keV}$. 


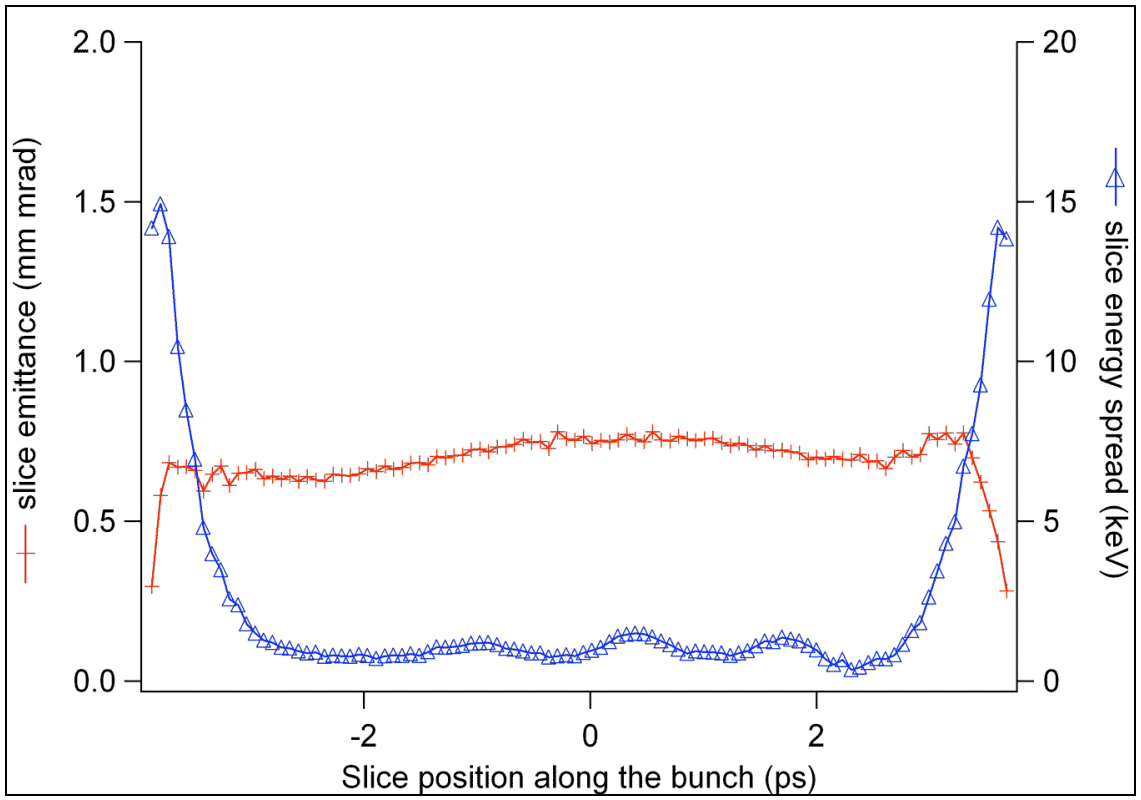

Figure 6.3: Slice emittance and slice energy spread along the bunch at the exit of the photoinjector exit; SOA at $1.4 \mathrm{~m}$ from the cathode.

In order to measure and control the quality of the beam extracted from the cathode, complete diagnostics instrumentation will be installed between the gun and the first booster section. This constitutes a critical issue in the space budget and it implies to move the matching point far away from the cathode. The Figure 6.4 shows the emittance compensation obtained by setting SOA at $1.6 \mathrm{~m}$ from the cathode and by slightly modifying the injector parameters setting.

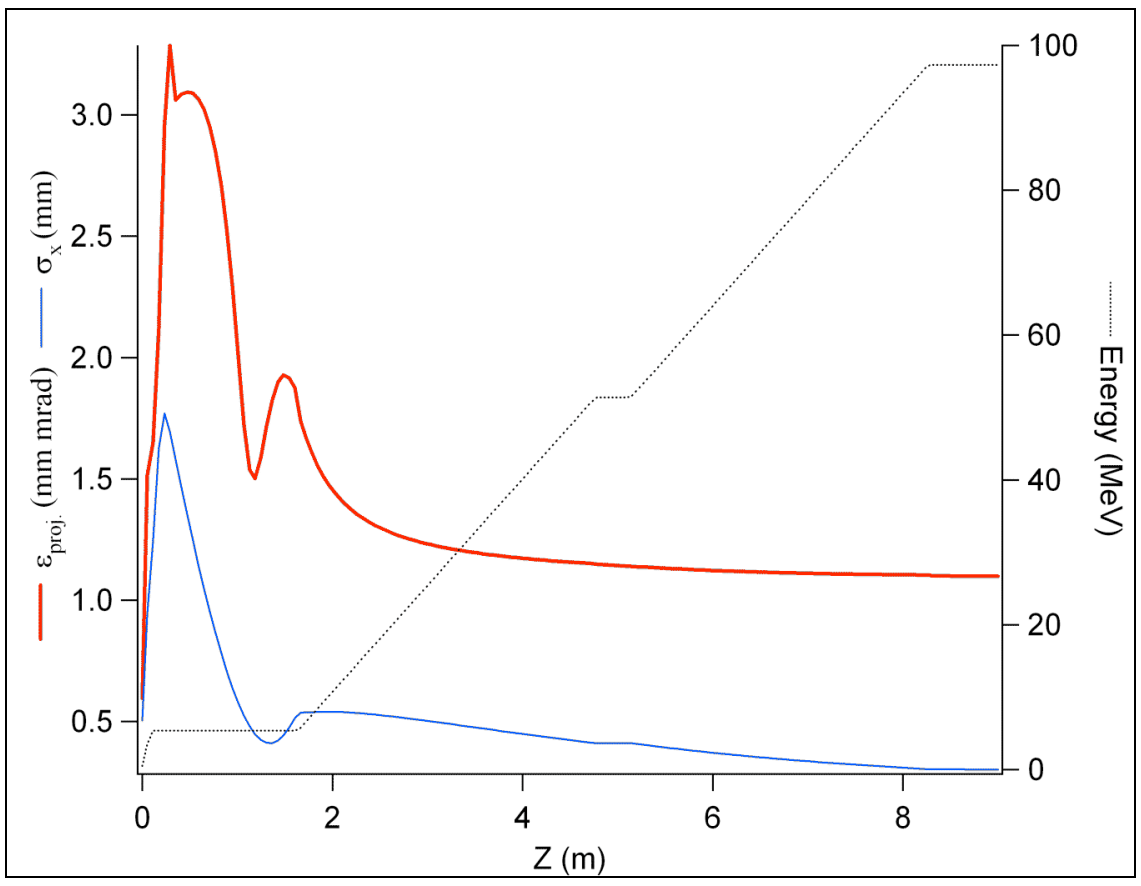

Figure 6.4: Projected radial emittance, radial spot dimension and energy along the photoinjector beamline for the short bunch regime; SOA at $1.6 \mathrm{~m}$ from the cathode. 
As expected, in this configuration the projected emittance increases to $1.1 \mathrm{~mm}$ mrad. The slice analysis of the bunch at the injector exit, Figure 6.5, reveals that the larger contribution to the projected emittance comes from the head and tail slices. Excluding the spikes, the projected emittance of $80 \%$ of the bunch is $0.90 \mathrm{~mm} \mathrm{mrad}$, with a slice emittance quite constant around $0.7 \mathrm{~mm}$ mrad.

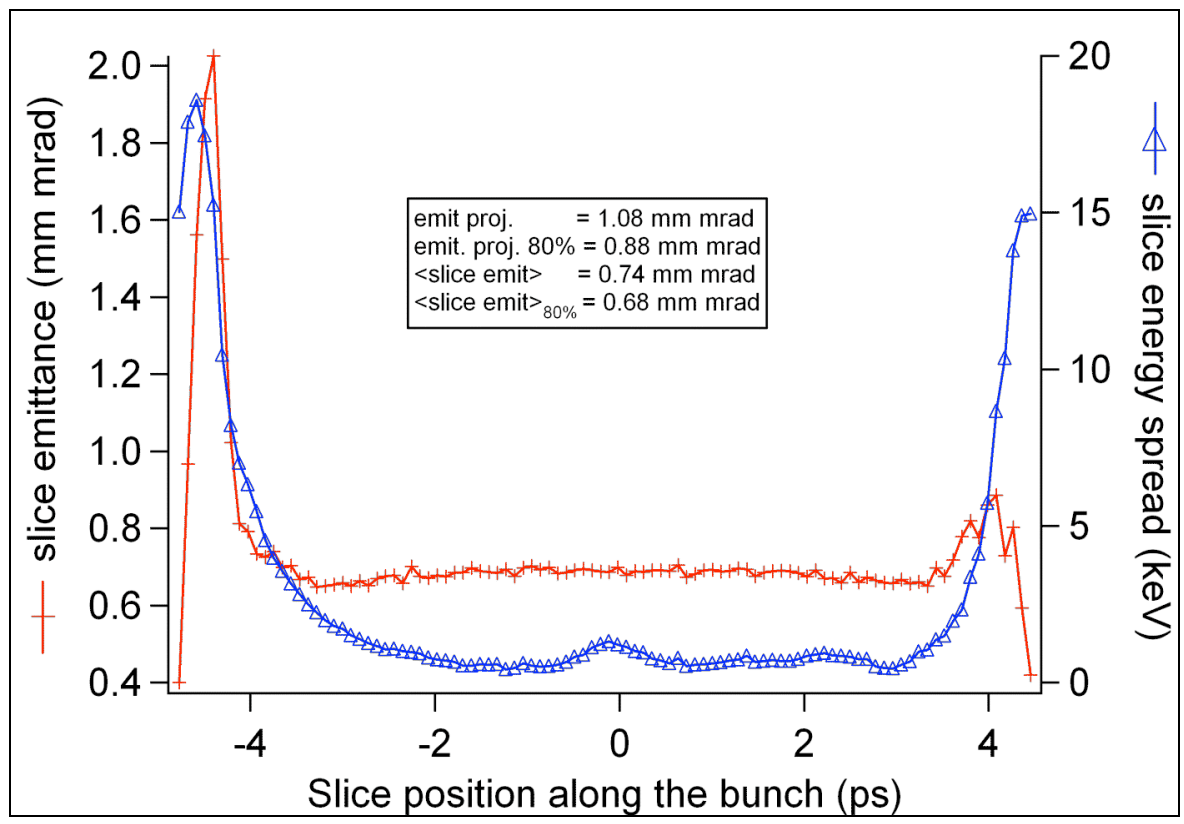

Figure 6.5: Slice emittance and slice energy spread along the bunch at the exit of the photoinjector exit; SOA at $1.6 \mathrm{~m}$ from the cathode.

\section{Medium Bunch}

\section{Flat-top current profile}

In the FEL, a seed laser pulse duration $100 \mathrm{fs}$ overlapping with an electron beam of $\sim 200$ fs bunch duration at the undulator entrance. Hence, timing synchronization is a critical issue. To relax the jitter tolerances, a 'medium' bunch case has been considered, consisting in a 600fs electron bunch with the same peak current ( 1kAmp). This is translated to an increase of the extracted charge up to $800 \mathrm{pC}$ and in a longer drive laser pulse at the cathode (9ps FWHM, with $0.5 \mathrm{ps}$ of rise/fall time). A peak accelerating gradient of $110 \mathrm{MV} / \mathrm{m}$ has been assumed and an optimization procedure similar to the short bunch case has been followed, considering a distance cathode-SOA of $1.6 \mathrm{~m}$ as a constraint. At the end of the photoinjector the medium bunch length is $10 \mathrm{ps}$ (FWHM) with a peak current $\sim 80$ Amps (Figure 6.6).

Longitudinal wakefields in the two accelerating sections has been included and Figure 6.7 shows how the longitudinal phase space is consequently modified. 


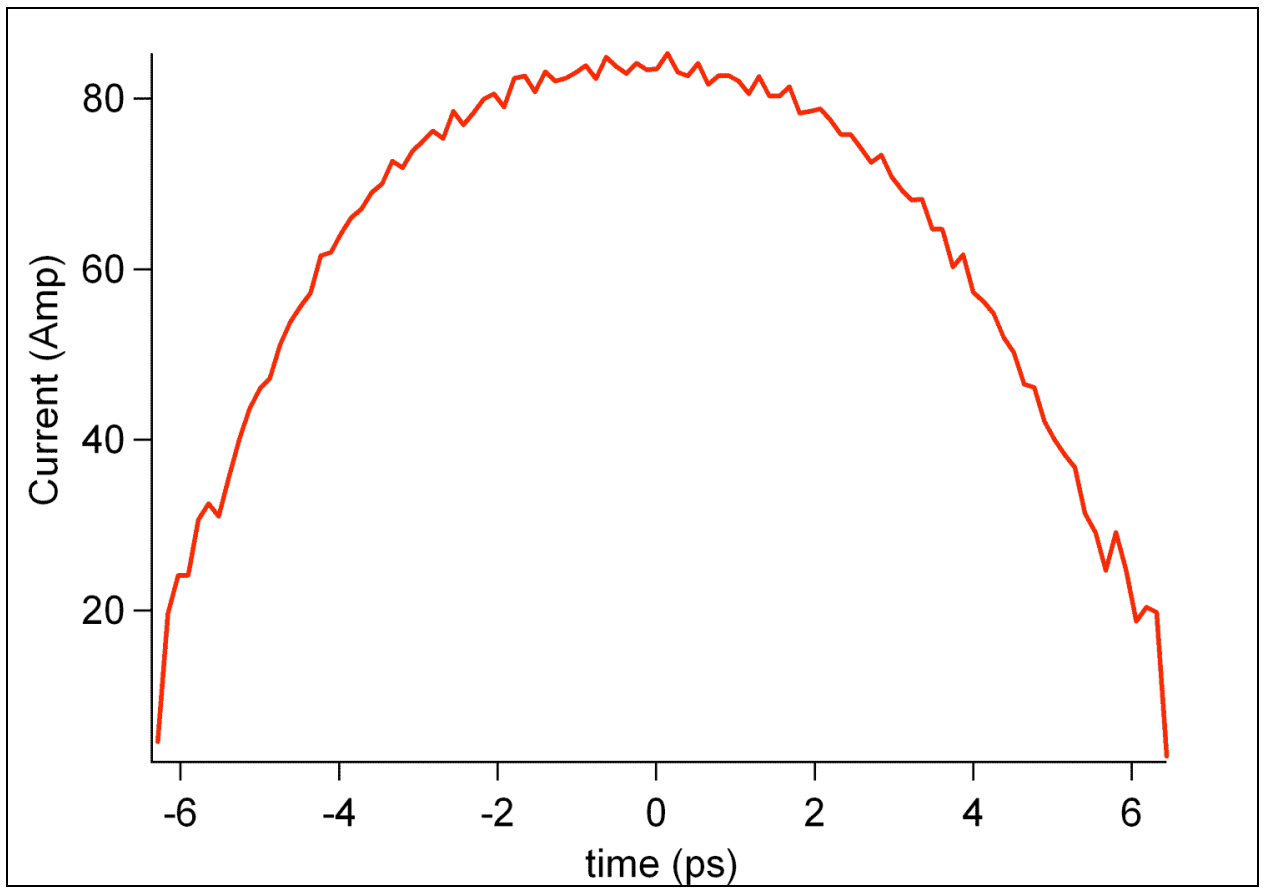

Figure 6.6: Current distribution of the medium bunch at the injector exit ( 95MeV).

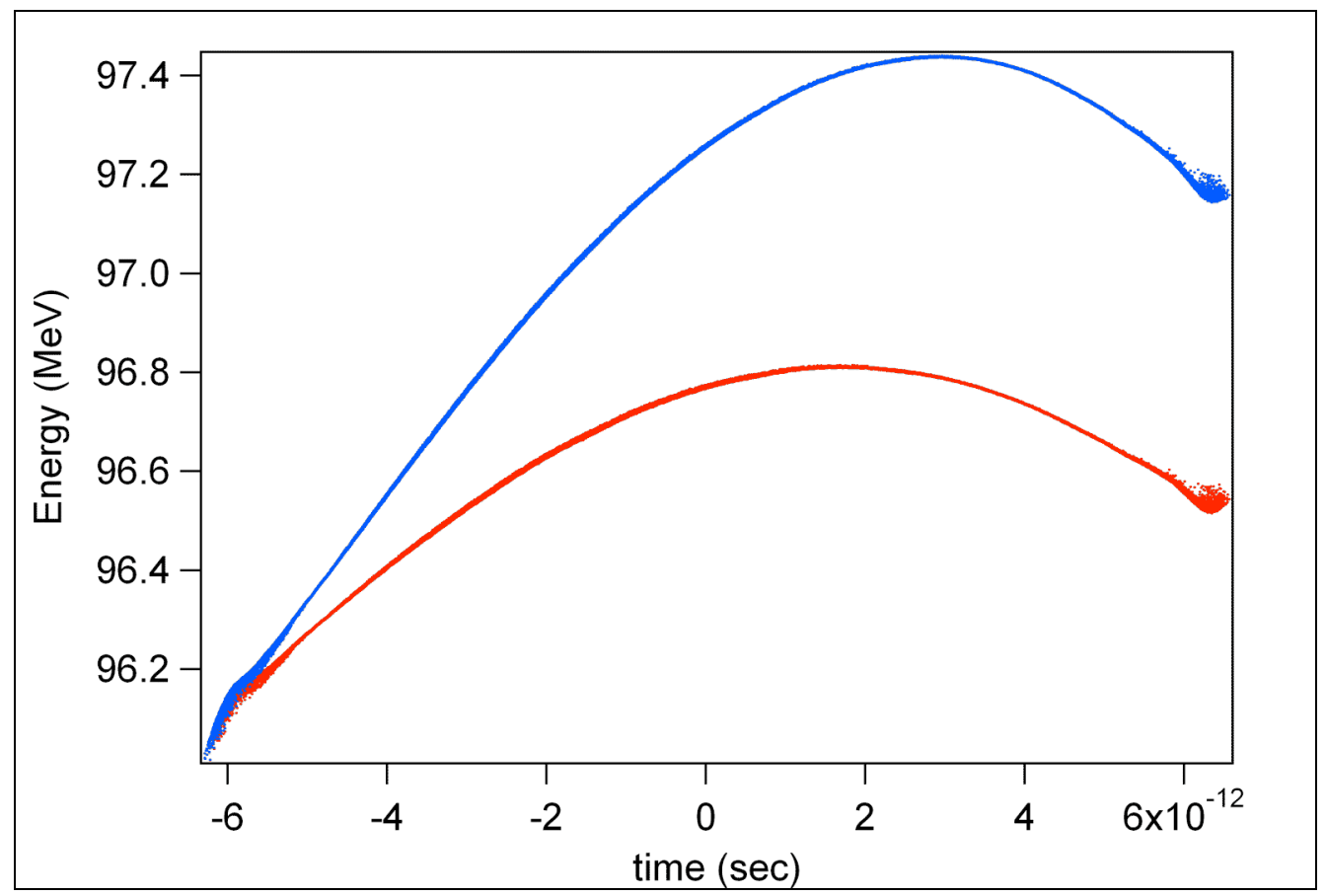

Figure 6.7: Longitudinal phase space at the photoinjector exit with (red line) and without (blue line) the contribution of the longitudinal wakefields in SOA and SOB.

A drive laser spot radius of $1 \mathrm{~mm}$ gives a thermal emittance contribution of $0.6 \mathrm{~mm} \mathrm{mrad}$, as in the short bunch. With the optimized injector tuning, the projected emittance at the photoinjector exit reaches $1.0 \mathrm{~mm}-\mathrm{mrad}$ (see Figure 6.8). 
ST/F-TN-06/11

LBNL - 60725

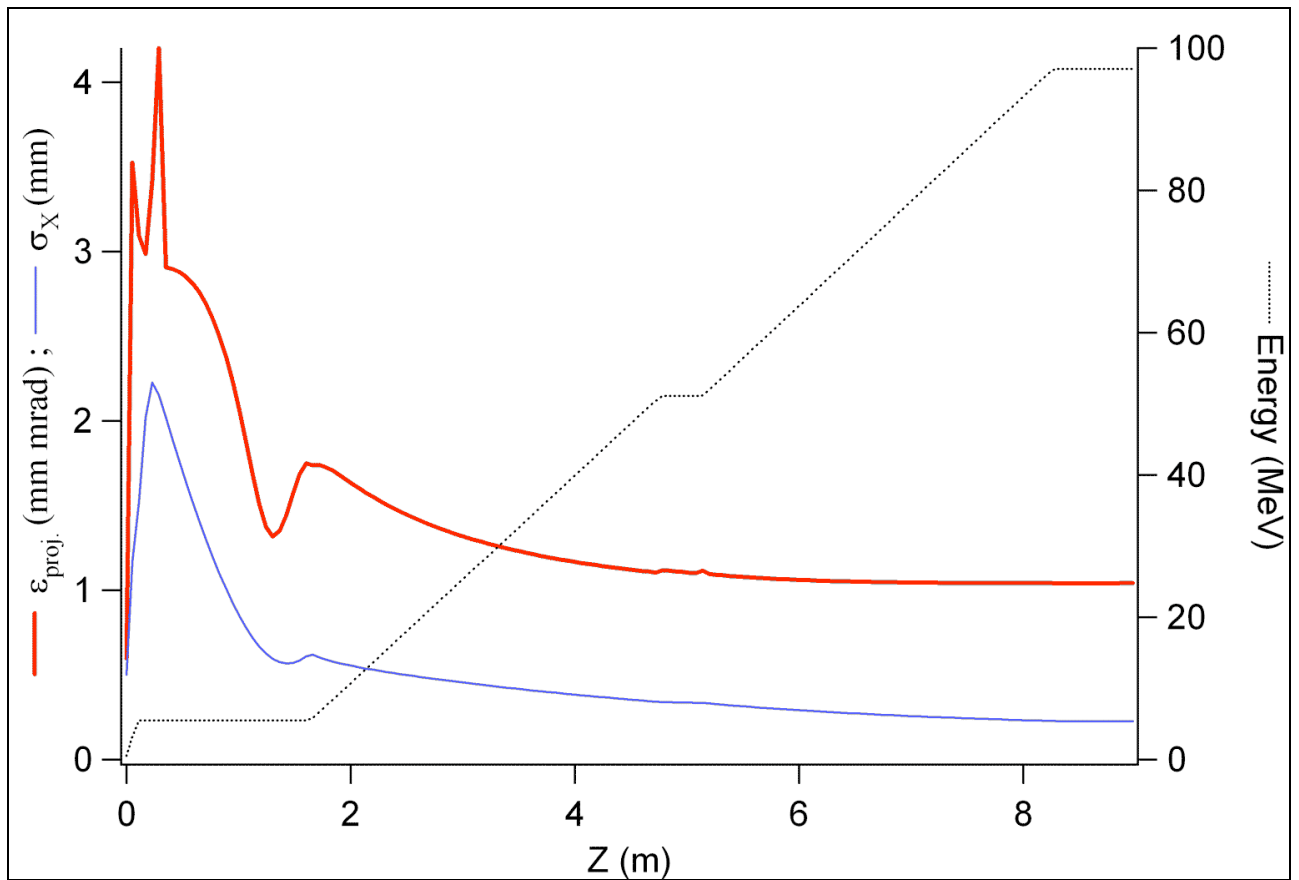

Figure 6.8: Projected radial emittance, radial spot dimension and energy along the photoinjector beamline for the medium bunch regime; SOA at $1.6 \mathrm{~m}$ from the cathode.

Figure 6.9 shows the slice emittance and the slice energy spread along the bunch. The average slice emittance is $\sim 0.7 \mathrm{~mm} \mathrm{mrad}$, with an average slice energy spread of $\sim 2 \mathrm{keV}$.

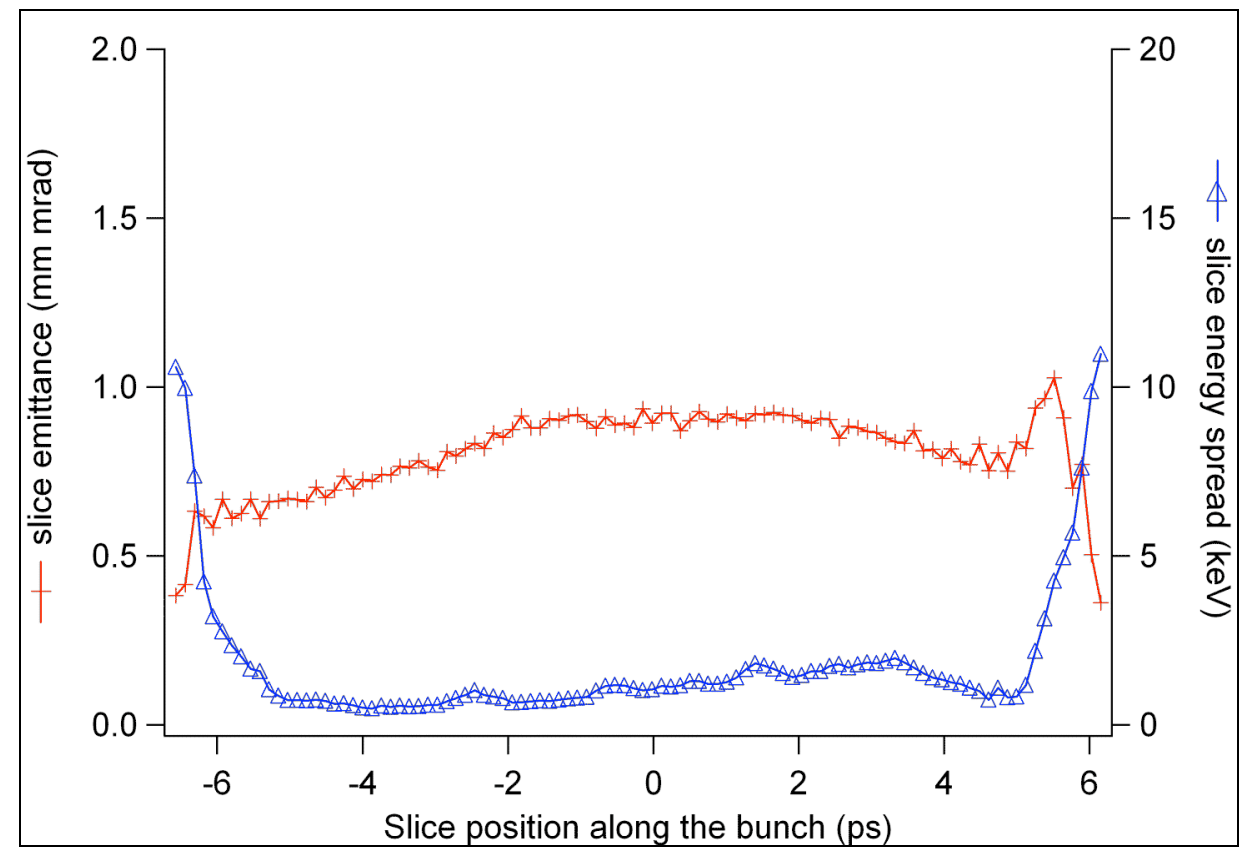

Figure 6.9: Slice emittance and slice energy spread along the bunch at the exit of the photoinjector exit; SOA at $1.6 \mathrm{~m}$ from the cathode. 


\section{Ramped current profile}

As described in the linac optimization Technical Note [6.6] a backward tracking simulation performed by LiTrack has revealed that a linearly ramped current distribution at the photoinjector exit represents a very interesting solution to linearize the wakefields fields in the Linac accelerating sections. Figure 6.10 shows the longitudinal phase space (Figure 6.10a) and the current profile (Figure 6.10b) required by the linac optimization studies at the exit of the photoinjector for the medium bunch case.

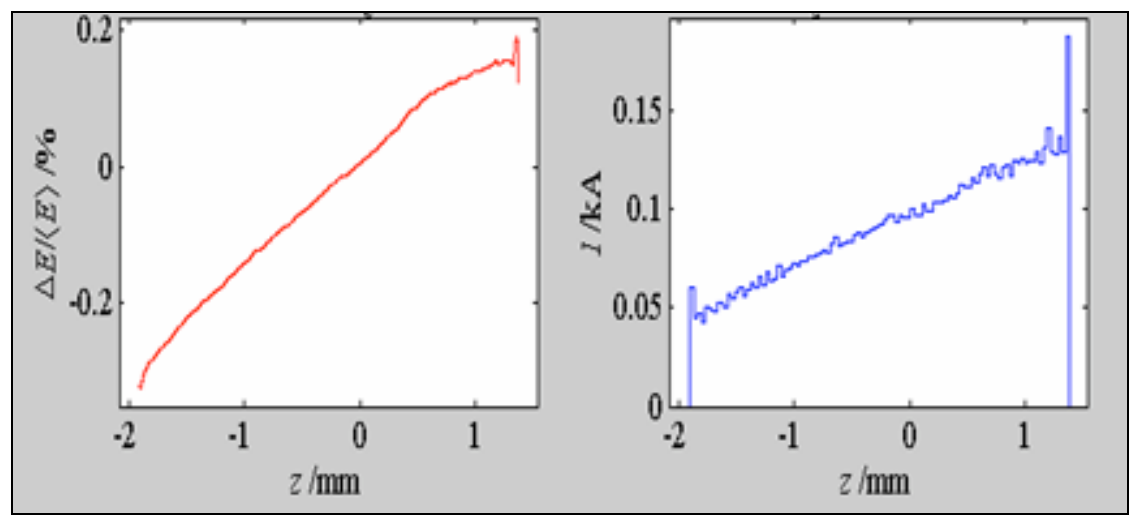

Figure 6.10: a) Longitudinal phase space and b) current distribution at the exit of the photoinjector for the medium bunch case (peak current on the bunch tail).

In order to satisfy these requirements, and to provide an injector output beam with a tailored current profile, we have studied how the initial profile of an emitted bunch evolves in the injector due to the longitudinal space charge field. To produce a final ramped current beam, a special initial profile has to be found that evolves in such a way to produce final desired shape.

To solve this problem, the longitudinal space charge fields at the cathode have been investigated, being the main responsible for the blowing out of the particles, especially in case of high peak current.

In case of a uniformly charged bunch of length $L$, the longitudinal space charge field on axis, inside and outside the bunch, at a distance $z$ from the bunch tail is given by the following equation:

$$
E_{Z}^{S C}(z)=\frac{Q}{2 \pi \varepsilon_{0} R^{2}} H(z),
$$

where $\mathrm{Q}$ is the total charge, $\mathrm{R}$ the bunch radius and $\mathrm{H}(\mathrm{z})$ is:

$$
H(z)=\sqrt{\left(1-\frac{z}{L}\right)^{2}+\left(\frac{R}{\gamma L}\right)^{2}}-\sqrt{\left(\frac{z}{L}\right)^{2}+\left(\frac{R}{\gamma L}\right)^{2}}-\left|1-\frac{z}{L}\right|+\left|\frac{z}{L}\right|
$$

Since the low energy of the electron bunch at the cathode, $\gamma=1$ is assumed. Figure 6.11 shows the field $\mathrm{E}_{\mathrm{z}}^{\mathrm{SC}}(\mathrm{z})$ versus $\mathrm{z}$ (normalized with respect to the bunch length $\mathrm{L}$ ) for a 
nominal $800 \mathrm{pC}$ bunch, with an edge radius of $1 \mathrm{~mm}$ and a bunch length of $3 \mathrm{~mm}$. The flattop current distribution at the cathode is deformed by this space charge field into a parabolic distribution after several centimeters, suggesting that a linear current distribution would suffer a strong degradation before entering into the relativistic regime. Thus the drive laser pulse should be shaped according to a non-linear distribution pattern.

The expression (6.2) has been generalized for an arbitrary current density distribution $\rho(z)$ at the cathode [6.7], obtaining:

$$
E_{Z}{ }^{S C}(z)=\int_{0}^{L} \frac{Q}{2 \pi \varepsilon_{0} R^{2} L} \cdot \rho\left(z^{\prime}\right)\left[\frac{z^{\prime}-z}{\sqrt{\left(z^{\prime}-z\right)^{2}+R^{2}}}-\frac{\left|z^{\prime}-z\right|}{z^{\prime}-z}\right] \cdot d z^{\prime}
$$

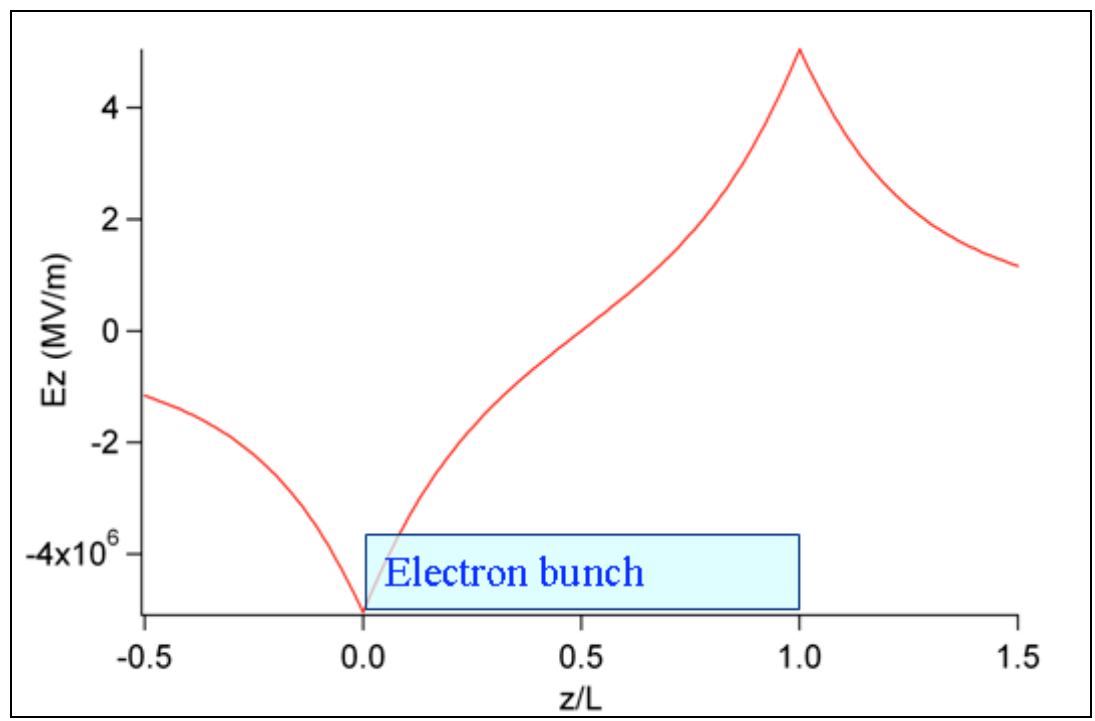

Figure 6.11: Longitudinal space charge field $(z=0)$ sampled by a flat top bunch at the cathode in the medium bunch case.

A large charge density close to the cathode surface increases the image charge field and this should be considered in the generation of a ramping charge distribution. The effect of the image charge at the cathode can be easily included by adding the field $\mathrm{E}_{\mathrm{z}}^{\mathrm{SC}}(-\mathrm{z})$ to the formula (6.3).

Expression (6.3) represents a useful analytical instrument to quickly predict the evolution and distortion of an arbitrary current profile. Figure 6.12 shows the space charge field inside a bunch having respectively a linear and a quadratic ramping in the current distribution (image charge not included). 


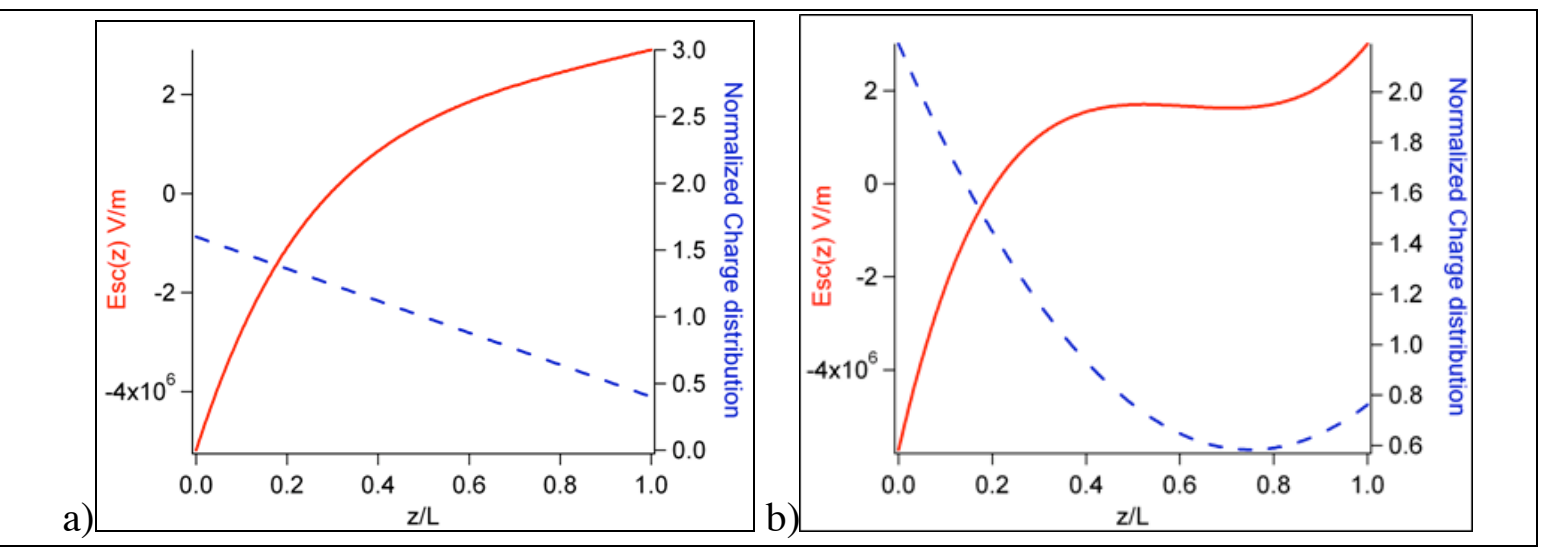

Figure 6.12: Longitudinal space charge field (red line) sampled at the cathode by a linear (a) and by a quadratic (b) ramp current distribution (blue line).

As anticipated, a linear ramp charge distribution samples an almost quadratic longitudinal space charge field (Figure 6.12a), which deteriorates the ramping current profile while propagating through the injector. Figure 6.13 shows that the original linear ramp has evolved into a parabolic-like distribution at the injector exit.
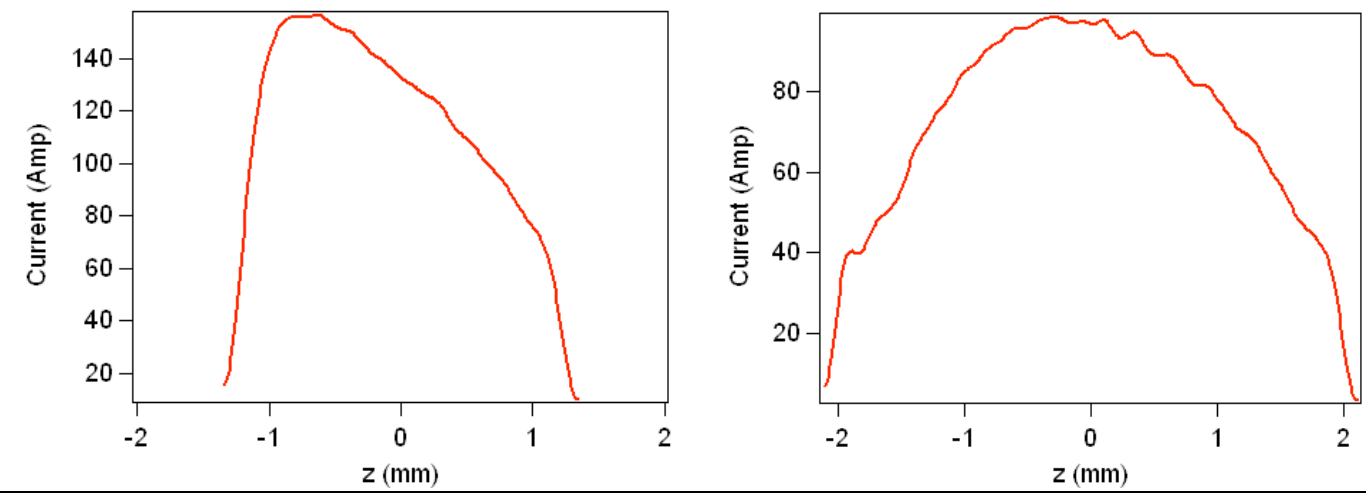

Figure 6.13: Linear ramp current distribution at the cathode (a) and the resulting profile at the exit of the injector (b) in case of an $800 \mathrm{pC}$-bunch.

Also a quadratic ramp, like Figure $6.12 \mathrm{~b}$, is strongly modified during the transport between the cathode and the SOA section, but the linearity of the space charge field at least in the middle of the bunch helps in preserving the ramp in about $70 \%$ of the bunch (see Figure 6.14). Even in this case the high peak current of the tail is reduced because of the spreading of the electrons suffering a strong backwards space charge field. 


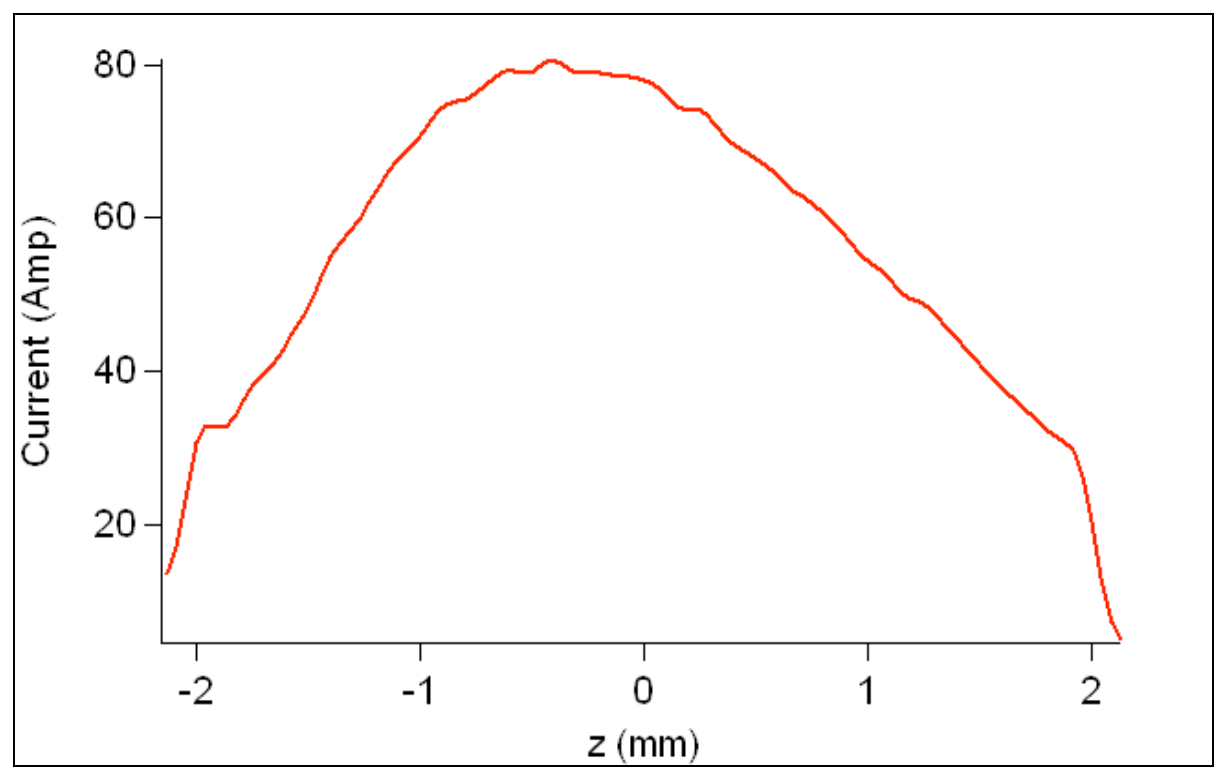

Figure 6.14: Current distribution at the end of the photoinjector of an 800pC-bunch, with a quadratic ramp (Figure 6.12b) at the cathode.

We have studied several initial current distributions in order to find the best one which linearizes as much as possible the space charge field experienced by electrons within the bunch. A fourth-degree polynomial distribution (see Figure 6.15) has been found to be an interesting solution that offers flexibility in compensating the high orders contributions of the space charge field (see Figure 6.16), and increasing the bunch fraction sampling a linear space charge field.

The current distribution plotted in Figure 6.15a has been considered as the baseline ramping distribution for the medium bunch case assuming a large efficiency in the laser pulse shaping process. However in a conservative scenario even a quadratic distribution could be used without severe drawbacks.

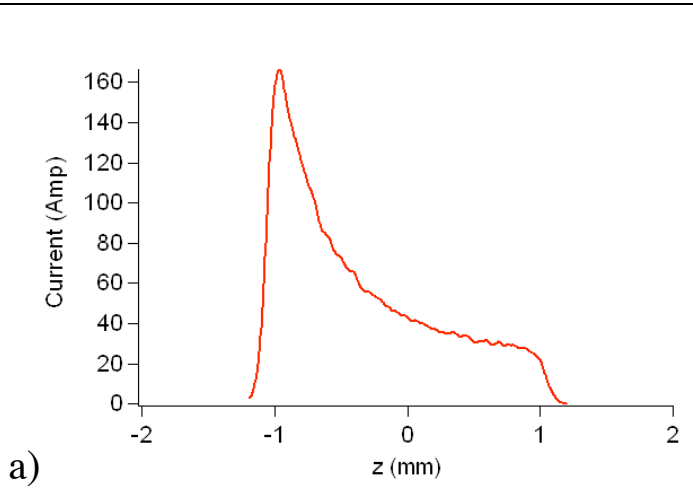

b)

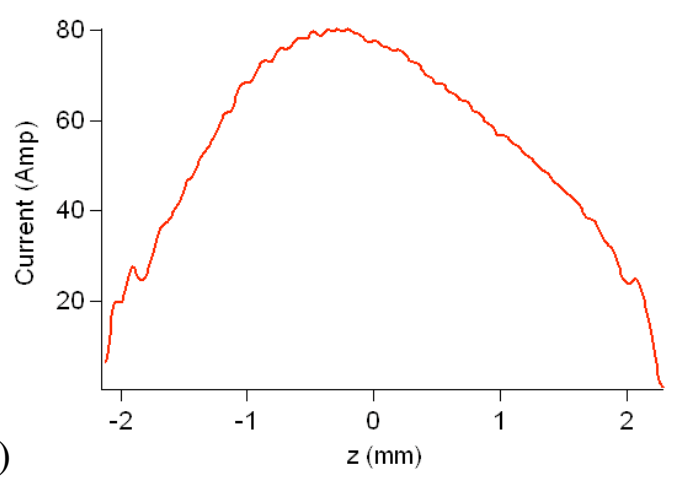

Figure 6.15: A fourth-degree polynomial distribution at the cathode (a) and at the end of the photoinjector $(b)$. 


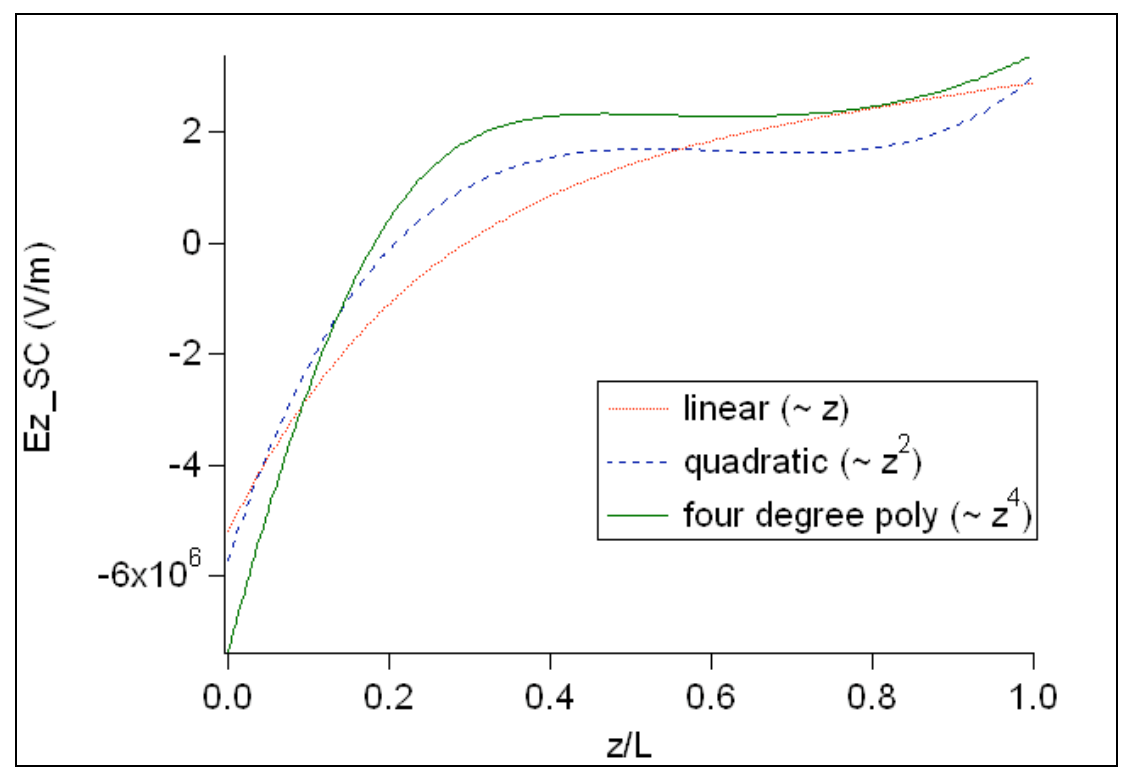

Figure 6.16: Comparison between the longitudinal space charge fields sampled by a linear, a quadratic and a fourth-degree polynomial distribution at the cathode.

Because of the non-uniform charge distribution of the ramping regime, it is very difficult to find an injector parameter set that completely satisfies the invariant envelope equation, performing perfect emittance compensation for all slices. Since each slice contains a different amount of charge, each slice evolves in a particular and unique way in the gunSOA drift when the injector parameters are fixed. Thus an average setting has been found that minimizes the projected emittance at the exit of the photoinjector (see Figure 6.17). The projected emittance at the injector exit reaches $\sim 1.39 \mathrm{~mm}$ mrad, while considering $80 \%$ of the bunch particles it is reduced to $1.21 \mathrm{~mm} \mathrm{mrad}$.

A slice analysis, as in the flat-top cases, has been performed and the results are shown in Figure 6.18. As expected, the ramping current distribution has an effect also in the slice emittance modulation along the bunch. The slice emittance of the minimum current areas (the head) is around $0.7 \mathrm{~mm} \mathrm{mrad}$, while in the peak current it reaches $1.1 \mathrm{~mm} \mathrm{mrad}$. Figure 6.19 shows the bunch top view at the injector exit: the transverse dimension is quite constant along $\sim 70 \%$ of the bunch. 
ST/F-TN-06/11

LBNL - 60725

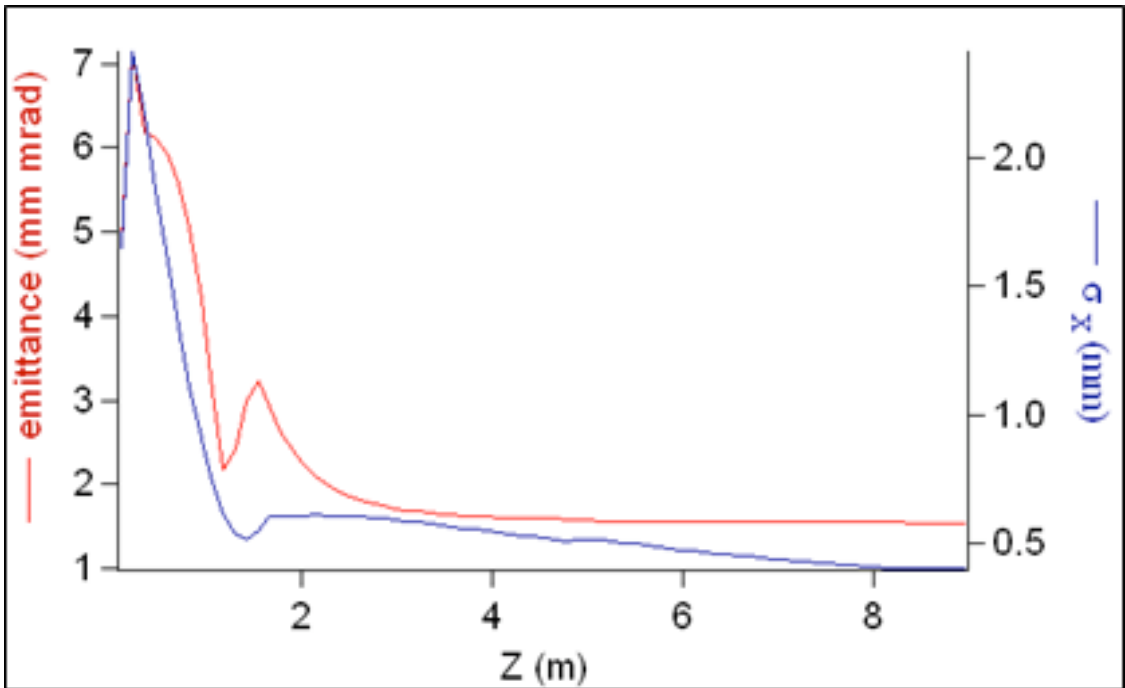

Figure 6.17: Projected Emittance (red line) and RSM transversal spot (blue line) evolution through the injector in the ramping medium bunch regime.

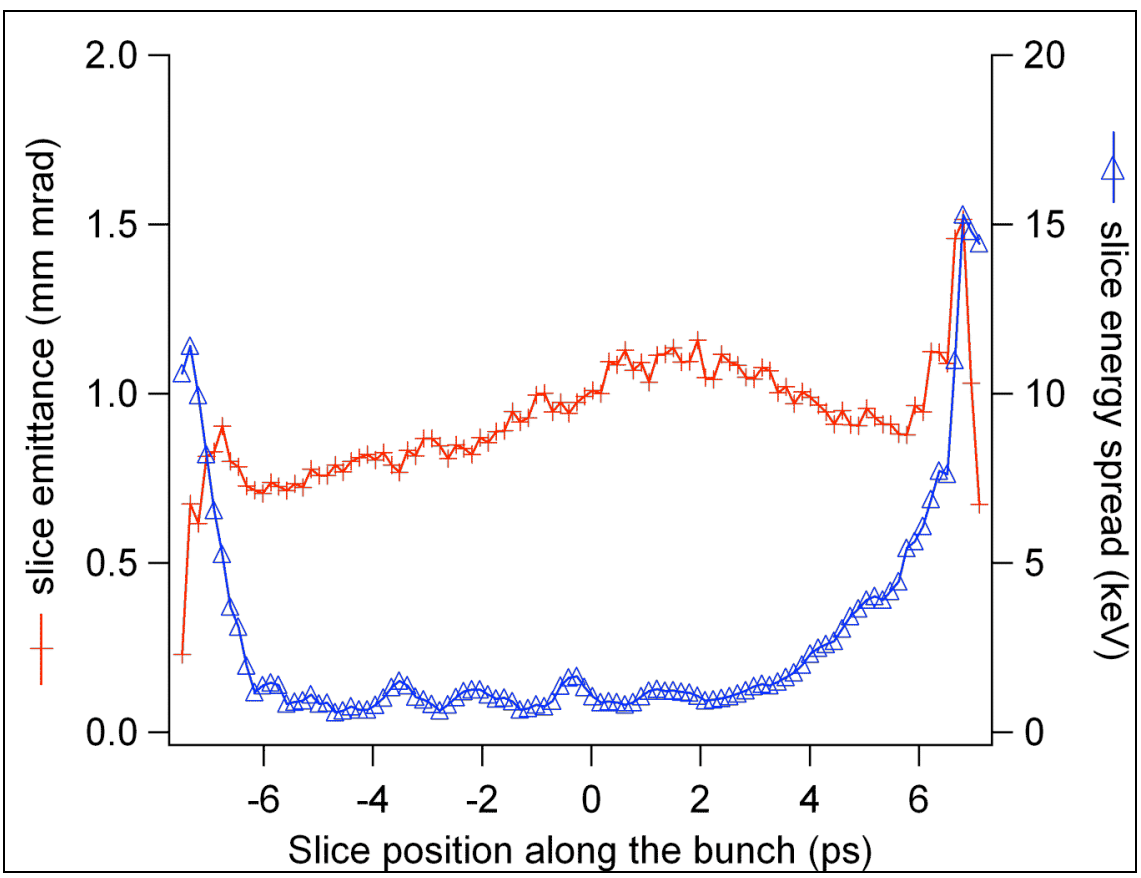

Figure 6.18: Slice emittance (red line) and slice energy spread (blue line) along the ramp medium bunch calculated at the exit of the photoinjector $(\sim 100 \mathrm{MeV})$. The head of the bunch is on the left. 


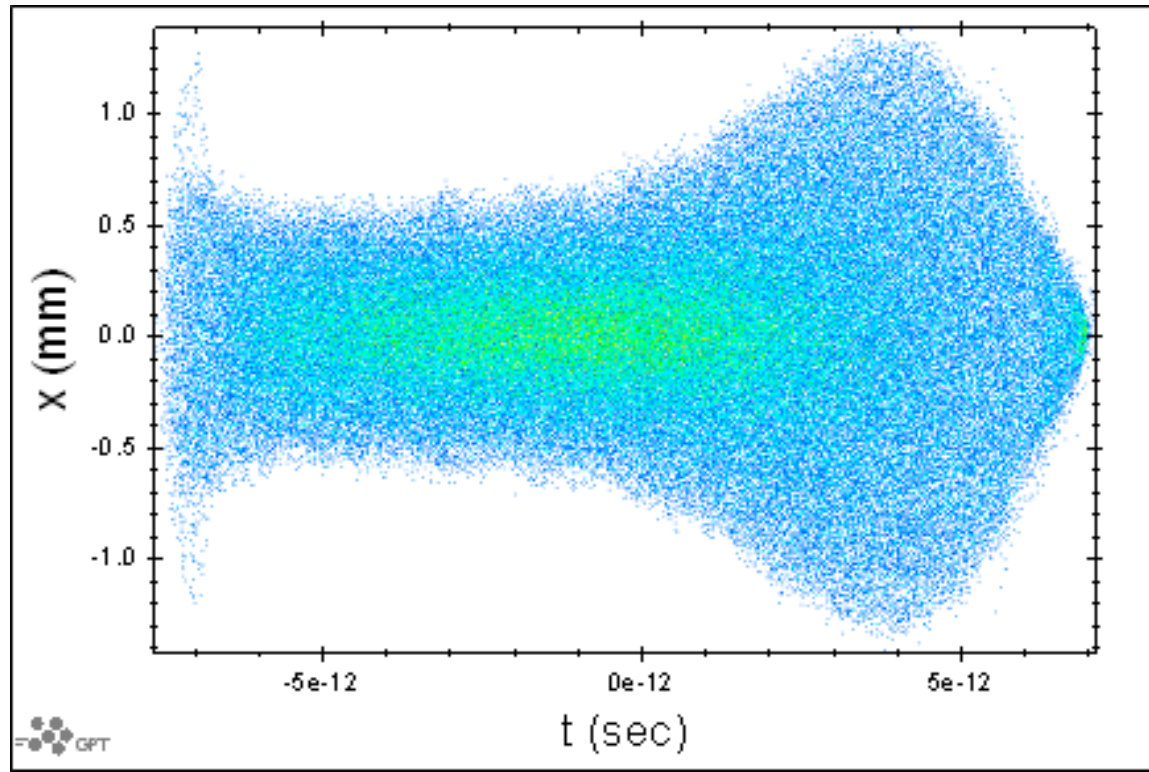

Figure 6.19: Top view of the ramp medium bunch calculated at the exit of the photoinjector. The head of the bunch is on the left.

\section{Long Bunch}

The 'long' bunch case represents the configuration at high bunch charge $(1 \mathrm{nC})$ and a relatively long drive laser pulse ( 10ps) at the cathode. This is a 'standard' case studied in several photoinjector labs [6.8] and we have tried to optimize our system configuration taking into account the beamline constrains, as previously mentioned. Limitation in peak accelerating field of the Gun $(110 \mathrm{MV} / \mathrm{m})$ and in position of the first booster section (distance between the cathode and the first cell center of the section: about $1.6 \mathrm{~m}$ ) have been assumed.

\section{Flat-top current profile}

The 'standard' flat-top (longitudinal) and top-hat (transverse) laser pulse shape is the first studied case. The simulation goal is to find an accelerator machine set-up in order to minimize the normalized rms transverse emittance of the output bunch. We begin the beam dynamics simulations at the cathode with a hard edge transverse profile with radius $\sim 1 \mathrm{~mm}$. The longitudinal profile is uniform with a rise/fall time of 0.6ps (see Figure 6.20). 


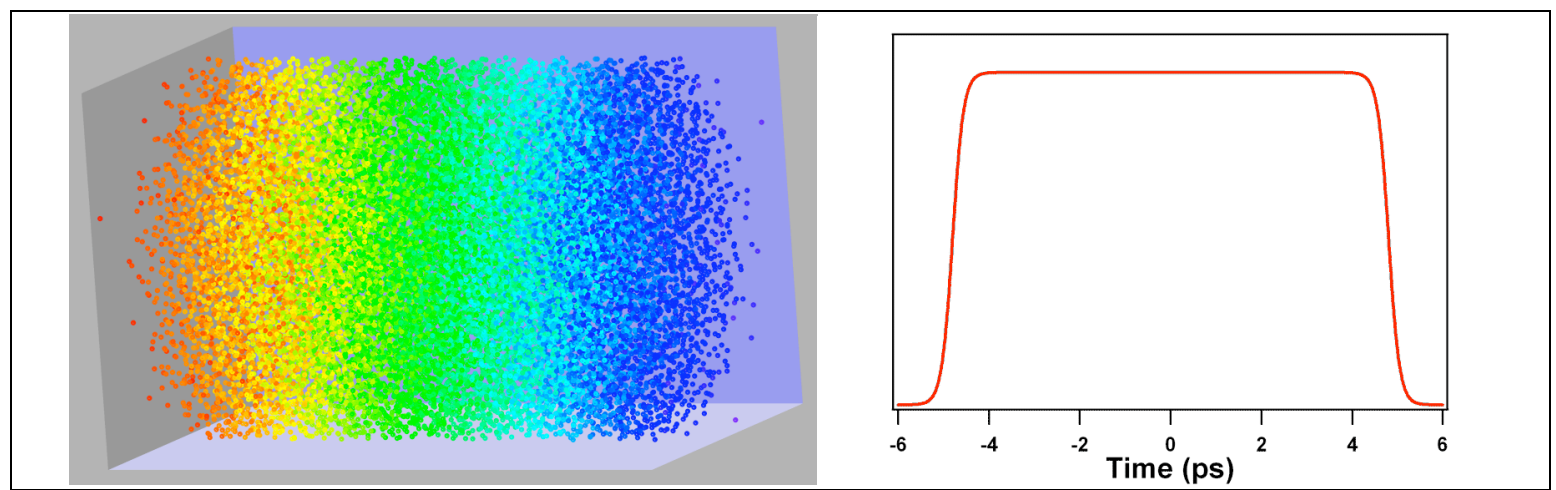

Figure 6.20: 3D particle plot at the cathode and its distribution.

As in the previous cases, by applying the emittance compensation procedure, an accelerator configuration has been found that minimizes the normalized projected emittance.

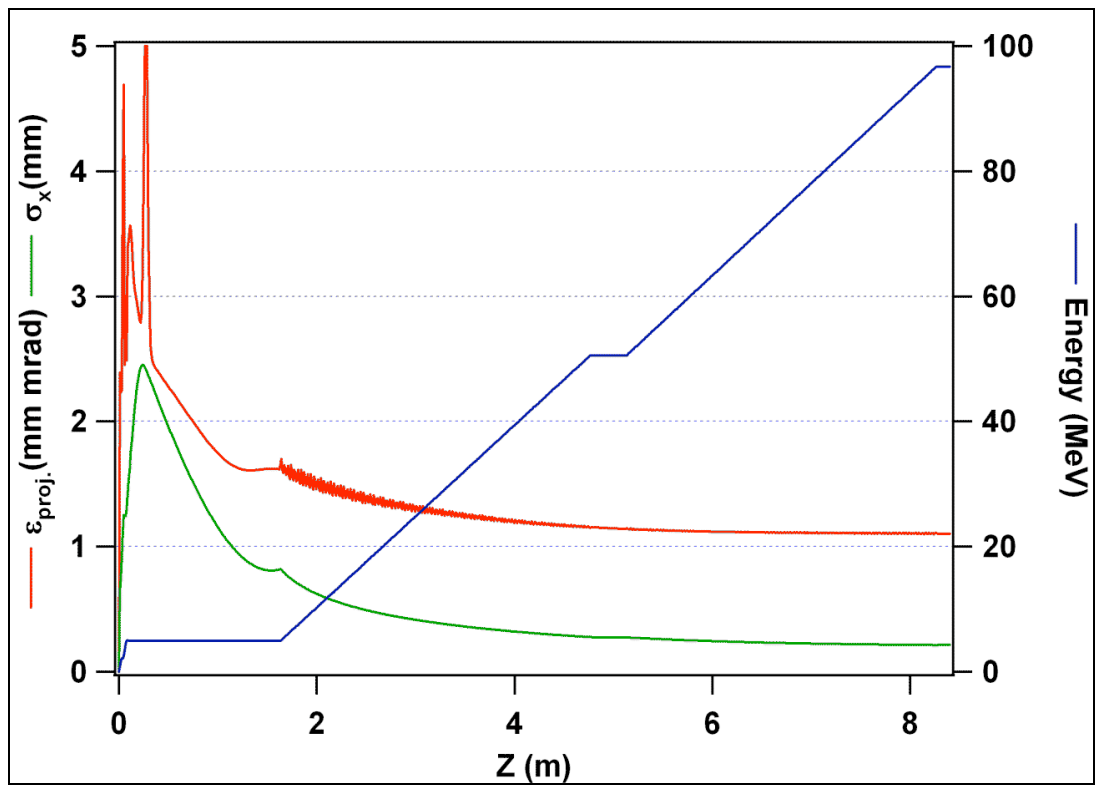

Figure 6.21: Beam envelope, emittance and energy versus $\mathrm{z}$.

For this case, a gun RF phase has been set as $25.2^{\circ}\left(90^{\circ}\right.$ on crest) and the solenoid magnet field of 2420 gauss. Figure 6.21 shows the beam envelope (green) and the emittance (red) along the injector and the final projected emittance value is $1.1 \mathrm{~mm} \mathrm{mrad}$.

To investigate the slice properties of the output beam, the bunch length was divided into 100 slices and we calculate the emittance and the energy spread of the macro-particles contained in each slice, obtaining the plot reported in Figure 6.22. The averaged slice emittance is $0.9 \mathrm{~mm}$ mrad, while the slice energy spread is in the hundreds of $\mathrm{eV}$. This low energy spread value is due to large number of macro-particles in the simulation (1 million in this case), as well as to the removal of the RF field induced curvature in the energy spread calculation. 


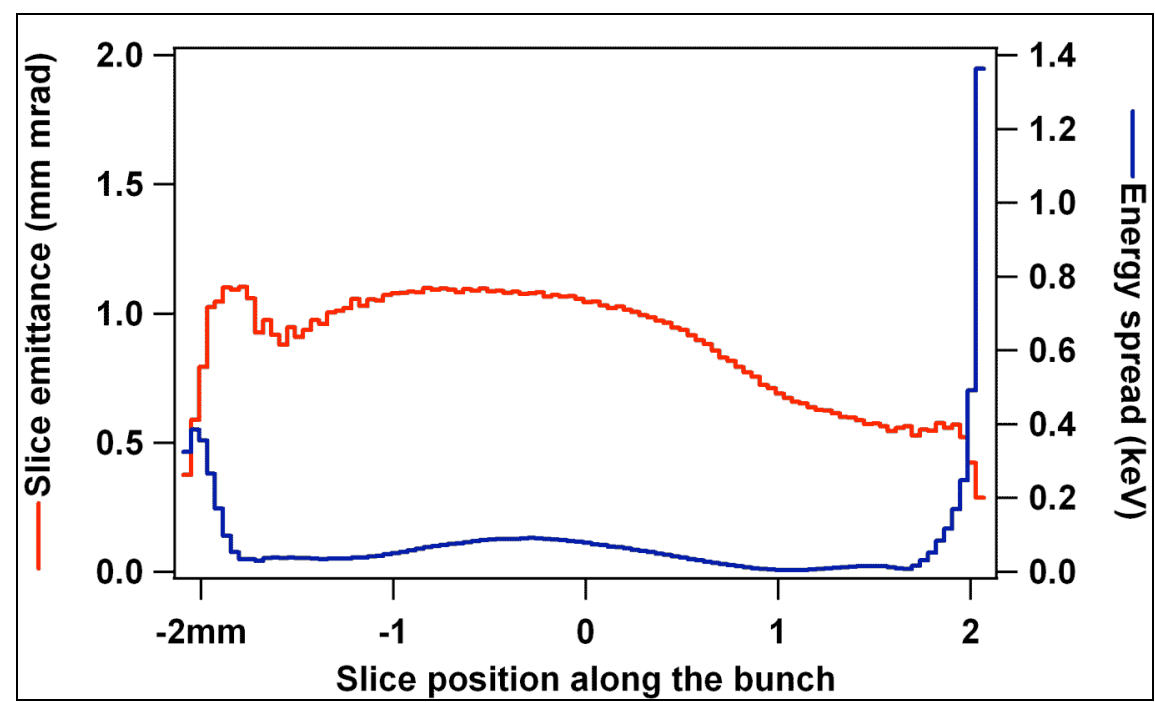

Figure 6.22: Slice emittance and energy spread along the bunch.

Figure 6.23 shows the bunch current profile and the longitudinal phase space, where also the effect of wakefields of the accelerating sections has been taken into account.

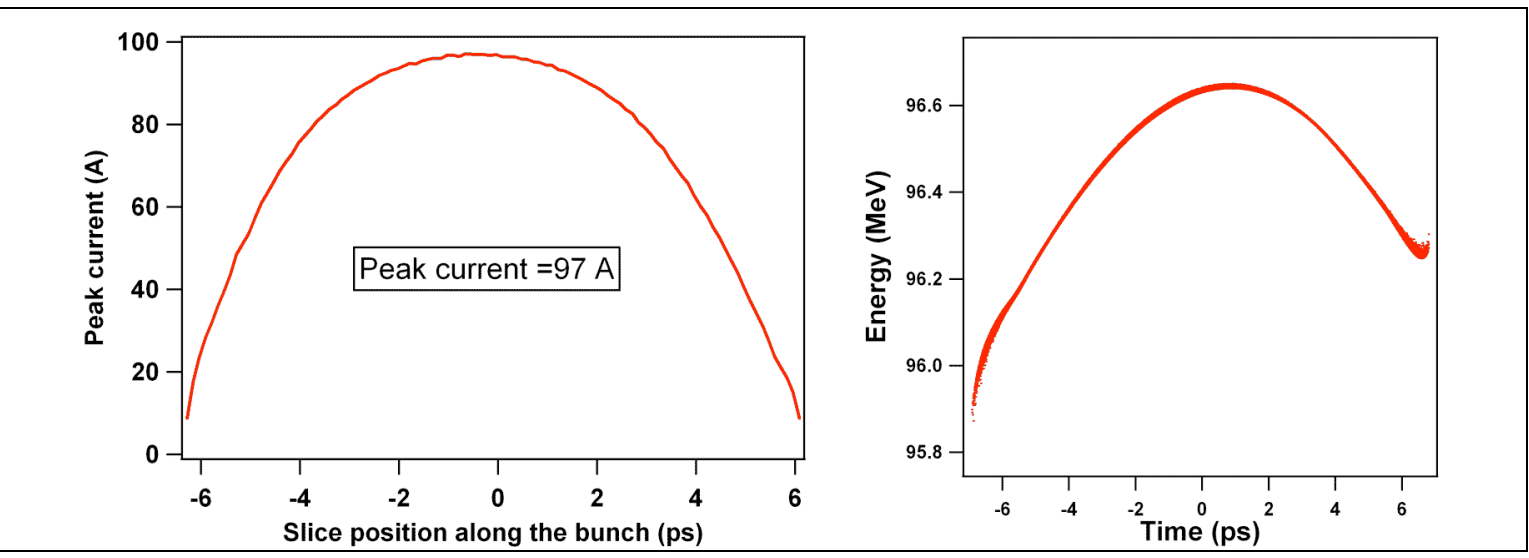

Figure 6.23 Current profile (left) and longitudinal phase space (right).

\section{Ramped current profile}

The possibility to produce an injector output beam with a ramped current profile has been considered even in the case of $1 \mathrm{nC}$ bunch charge. As in the previous case, the charge density inside the standard bunch shape has been changed, while keeping the $1 \mathrm{~mm}$ radius top-hat transverse dimension.

Due to the higher bunch charge value a different laser shape has been chosen with respect to the ramped medium bunch. A particle density distribution with less charge unbalanced between the head and the tail of the bunch has been preferred. This is shown in Figure 6.24 . 


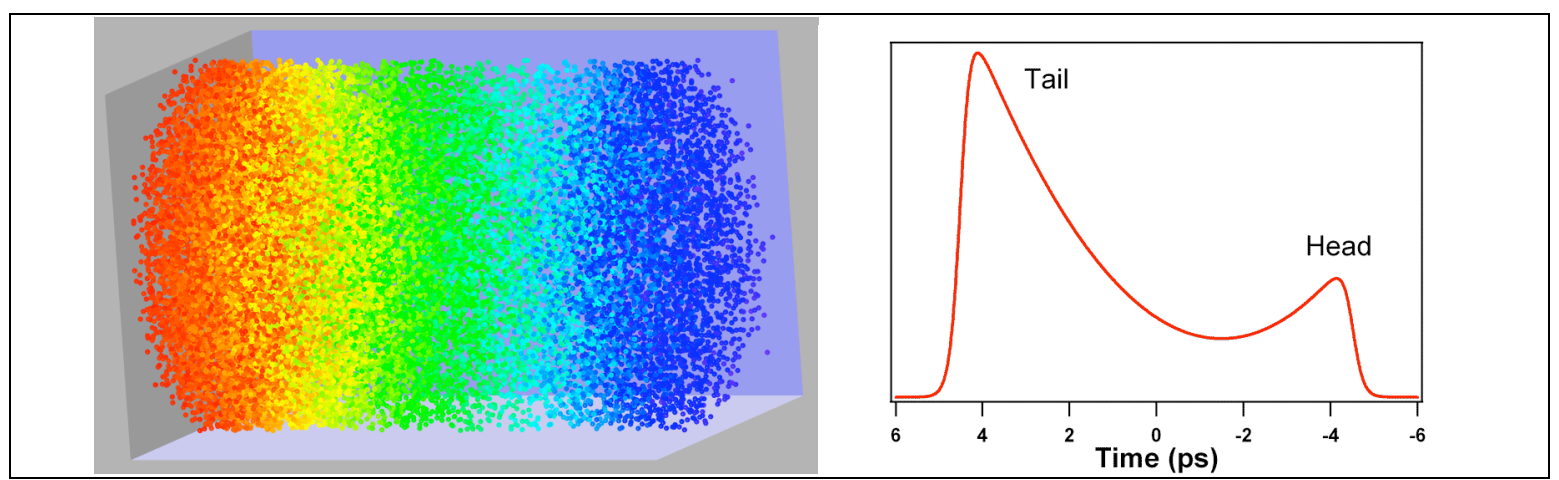

Figure 6.24: 3D particle plot at the cathode and its distribution.

The emittance compensation is less effective (see Figure 6.25) so the final projected emittance is $1.26 \mathrm{~mm}$ mrad at $97 \mathrm{MeV}$, with a $29.4^{\circ}$ Gun RF phase $\left(90^{\circ}\right.$ on crest) and the solenoid magnet field of 2450 gauss. Even if this value is slightly higher than the corresponding flat-top case, it is acceptable considering the benefit coming from the operation with a ramped current distribution, as shown in Figure 6.26.

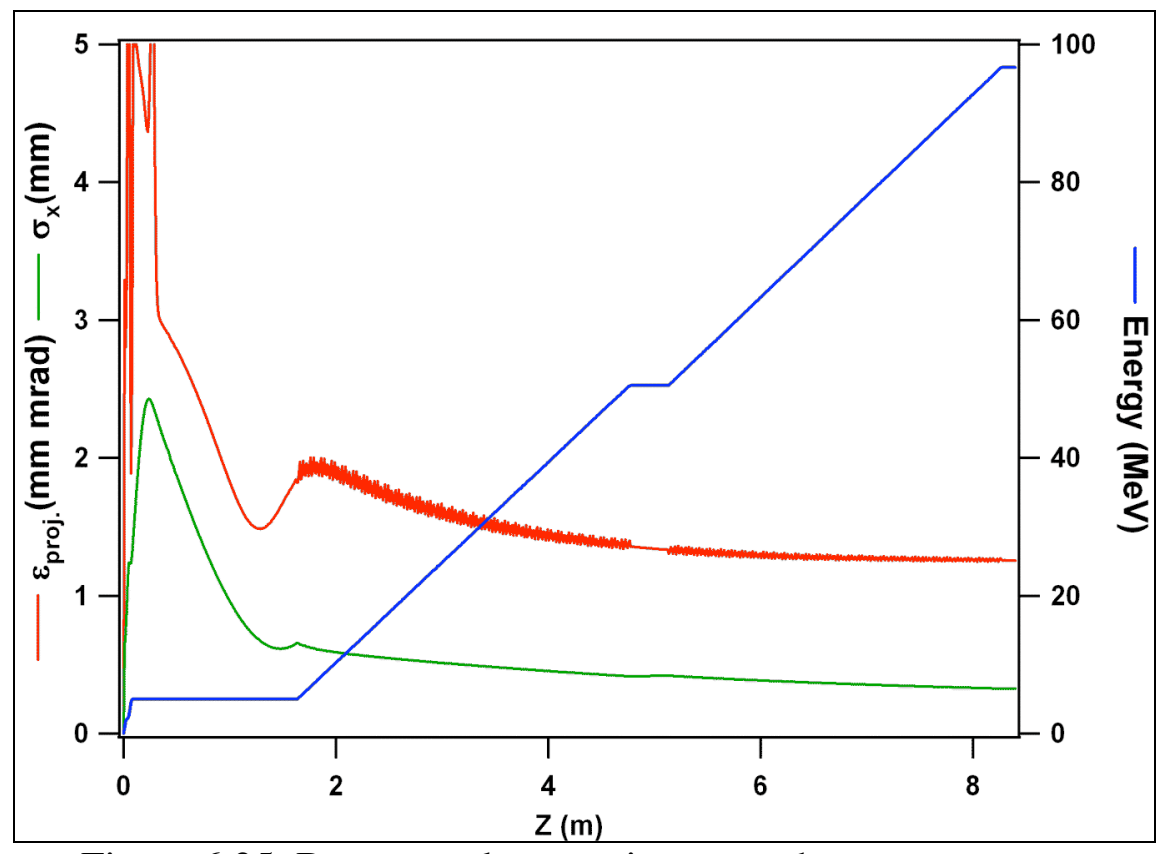

Figure 6.25: Beam envelope, emittance and energy versus $z$. 

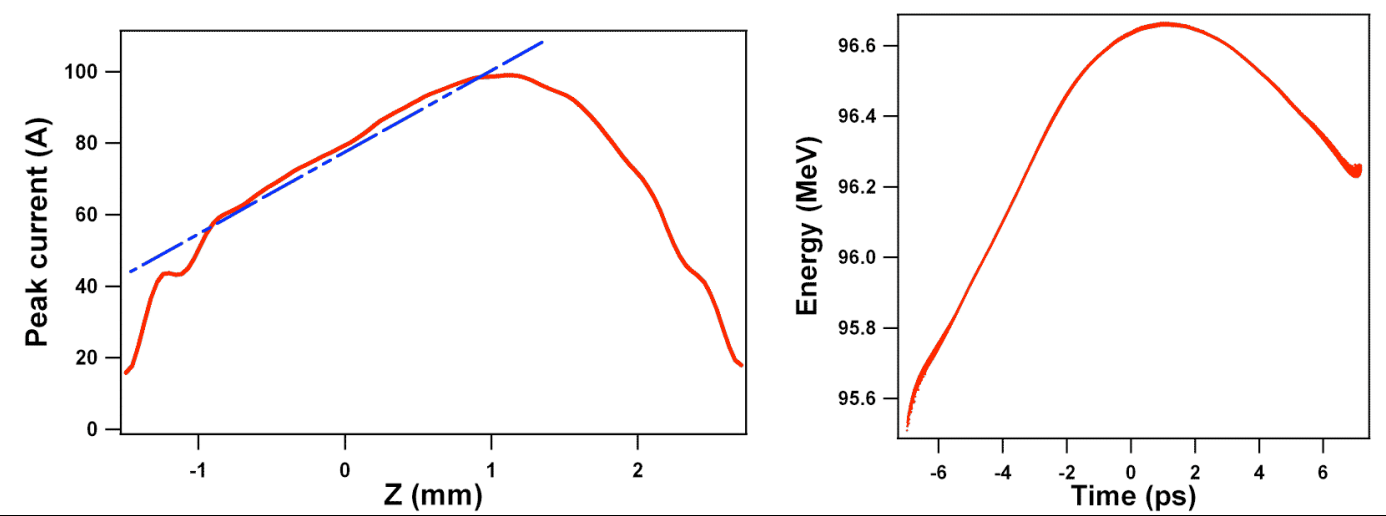

Figure 6.26: Current profile (left) and longitudinal phase space (right).

The bunch slice properties are shown in the Figure 6.27.

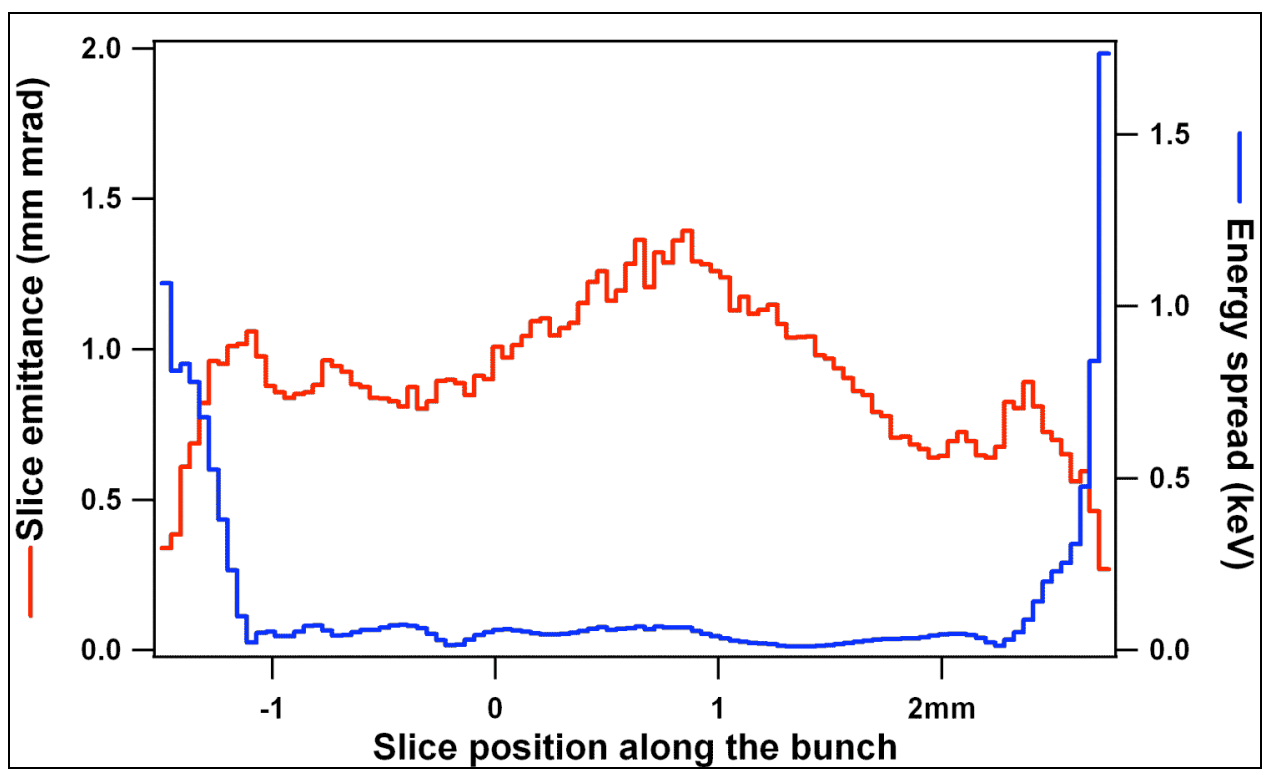

Figure 6.27: Slice emittance and energy spread along the bunch.

\subsection{Parametric sensitivity studies}

\section{Parameter choices and tolerances}

We have studied the stability and the robustness of the baseline injector solutions previously proposed. The characteristic variations of the output bunch parameters as a function of the input parameters have to be carefully estimated, because a seed harmonic cascade FEL is very sensitive to shot by shot stability. The temporal stability of the electron bunch at the entrance of the undulator, for example, is an important operational requirement to guarantee a reliable synchronization with the seed laser. Moreover the energy stability of the electron bunch is an issue for the stability of the FEL radiation 
itself, so it is important to investigate the contribution to the energy jitter coming from injector parameters jitters.

For these reasons the time of flight, the energy, the energy spread, the peak current and the emittance at the end of the injector have been identified as the main output parameters whose shot by shot variation should be quantified, as well the slice properties of the bunch.

Moreover, the strong correlation between the various injector parameters introduced by space charge effects does not allow considering each of them separately, but it is necessary to evaluate complete tracking simulations including all of the parameter jitter. By randomly sampling each injector parameter in a tolerances range fixed by the present technology a large number of injector cases have been tracked with GPT and ASTRA, obtaining a statistical evaluation of the expected jitters. In Table 6.1 the expected RMS jitter relative to the injector parameters are reported. Concerning the solenoid strength, the very high stability of the power supply allows us to neglect this parameter in the tolerance budget calculation based on randomly sampling all the others injector parameters. The sensitivity of the solenoid strength to the output jitters has been studied for all the bunch regimes.

Table 6.1: Expected rms variation of the injector parameters.

\begin{tabular}{|r|c|}
\hline $\begin{array}{c}\text { Injector } \\
\text { parameter }\end{array}$ & $\begin{array}{c}\text { Expected RMS } \\
\text { variation }\end{array}$ \\
\hline RF injection phase & $0.1 \mathrm{deg}$ \\
\hline Laser Arrival time & $100-300 \mathrm{fs}$ \\
\hline Gun $\mathrm{E}_{\mathrm{acc}}$ & $0.25 \%$ \\
\hline Solenoid strength & $0.005 \%$ \\
\hline SOA-SOB E $\mathrm{acc}$ & $0.25 \%$ \\
\hline SOA-SOB RF phase & $0.1 \mathrm{deg}$ \\
\hline Charge (laser pulse energy) & $4 \%$ \\
\hline Laser spot size & $4 \%$ \\
\hline Laser pulse length & $5 \%$ \\
\hline
\end{tabular}

As the sensitivity studies have demonstrated, the laser time jitter has been identified as the main component for the time jitter of the bunch at the injector exit, which strongly affects the energy stability at the end of the linac [6.6]. A conservative value of $300 \mathrm{fs}$ has been considered in order to identify the upper limit of the expected output jitter. As shown below, in some baseline configurations this value should be decreased to $\sim 100 \mathrm{fs}$ in order to guarantee the required stability of the FEL radiation output. 


\section{Short Bunch}

Considering the short bunch configuration (bunch charge 330pC, laser pulse duration $\sim 4.5 \mathrm{ps}$ ) we have tracked hundreds runs around the optimum layout, performing random gaussian sampling of each parameter with an RMS jitter given by the Table 6.1. Of course for a reliable statistics the number of runs should be increased more and more, but we have verified that the RMS value converges very quickly, allowing us to obtain a confident result already with 100 runs. Assuming an RMS laser time jitter of 300fs we obtain a RMS time jitter at the exit of the photoinjector of about 358fs (see Figure 6.28). This jitter value is larger than what we obtain from random uncorrelated effects (about 200fs), as shown in [6.9]. Hence, the correlation effects are very strong in this space charge dominated regime and a realistic evaluation of all the jitters should be performed taking in account a simultaneous variation of the parameters.

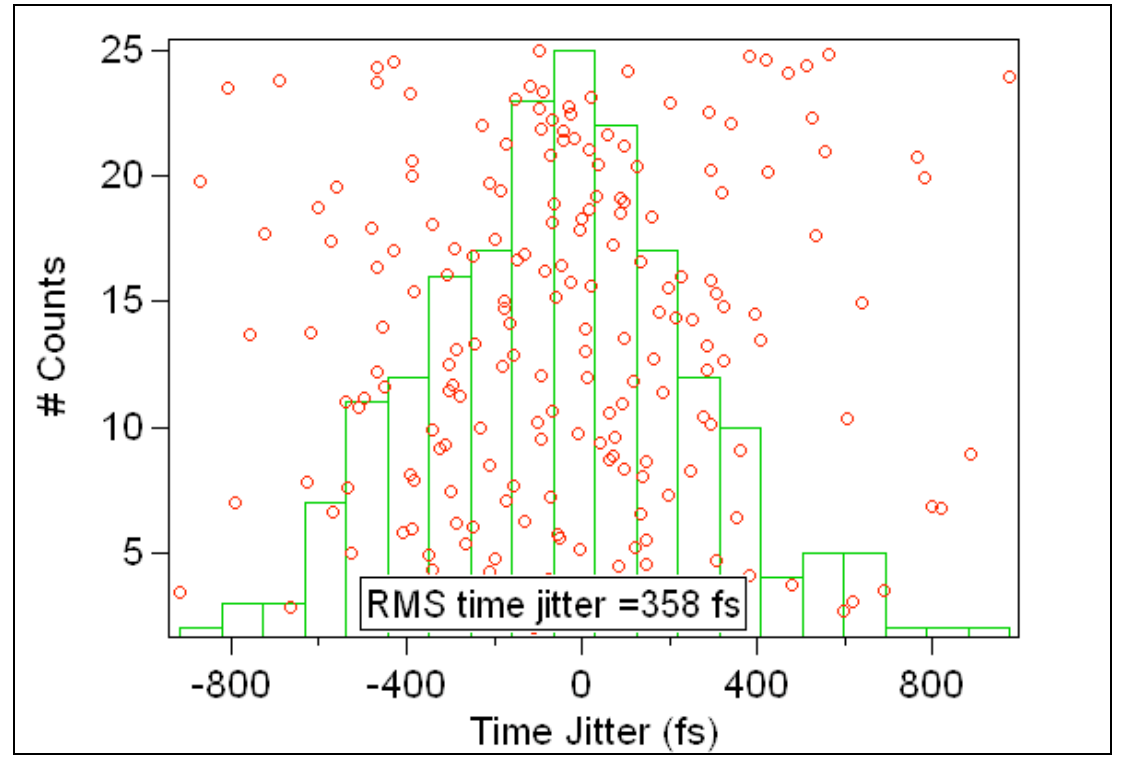

Figure 6.28: Time jitters results for the short bunch case.

The time jitter at the exit of the photoinjector is translated to a time jitter at the exit of the linac, i.e. after bunch compression, of $\sim 150 \mathrm{fs}$ (RMS) considering the tolerances budget of the linac itself [6.6]. As anticipated, due to this large time jitter, the short bunch regime is suitable only for a FEL seeded scheme with a long laser pulse (at least 600fs).

In order to estimate the current jitter it is necessary to define an equivalent current that is independent from the longitudinal bunch profile. At this purpose, the RMS bunch length, $\sigma_{t}$, at the exit of SOB has been calculated. The equivalent current $\hat{I}$ has defined as the current of a uniform distribution having $\sigma_{t}$ as RMS bunch length (see Figure 6.29), as given by:

$$
\hat{I}=\frac{Q}{\sqrt{12} \sigma_{t}},
$$

where $\mathrm{Q}$ is the total bunch charge. 
We have referred to the uniform distribution, but this treatment is absolutely general and it is possible to use whatever distribution (gaussian, parabolic, quadratic, etc...) as the reference, with $\hat{I}$ the corresponding peak current.

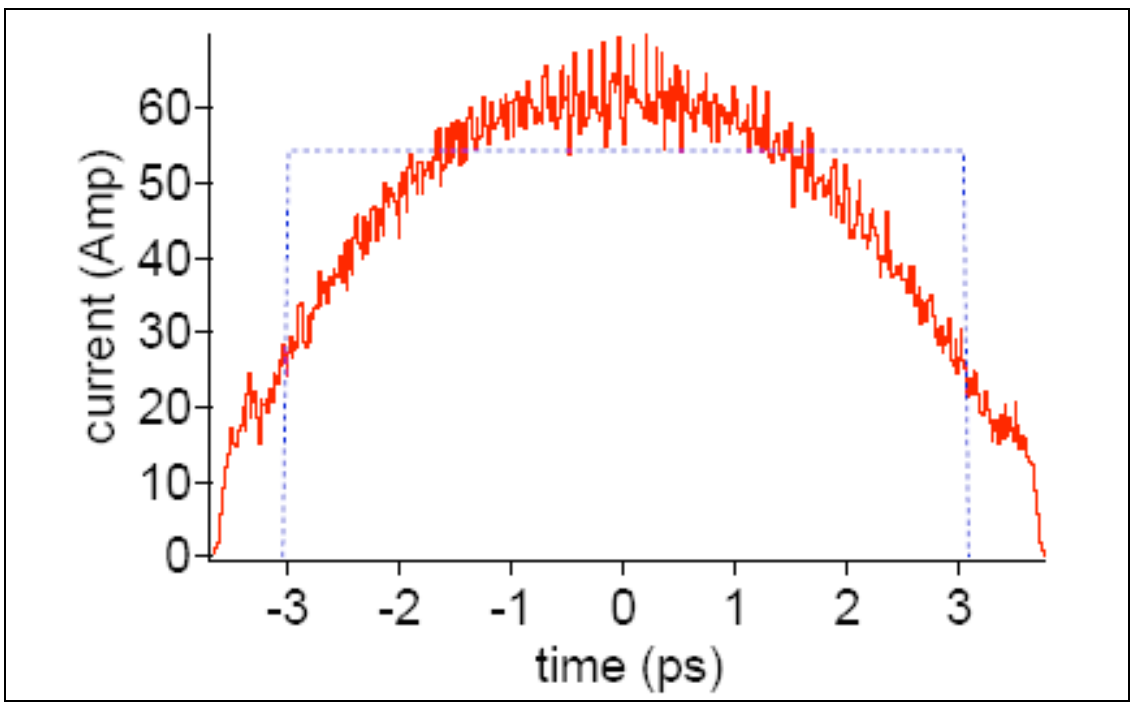

Figure 6.29: The red line corresponds to the bunch profile at the exit of SOB, while the blue dotted line is the correspondent equivalent square distribution.

Figure 6.30 shows the simulation results over hundreds of runs, considering again a 300fs laser time jitter: a RMS current jitter of $\sim 2.9 \%$ is obtained.

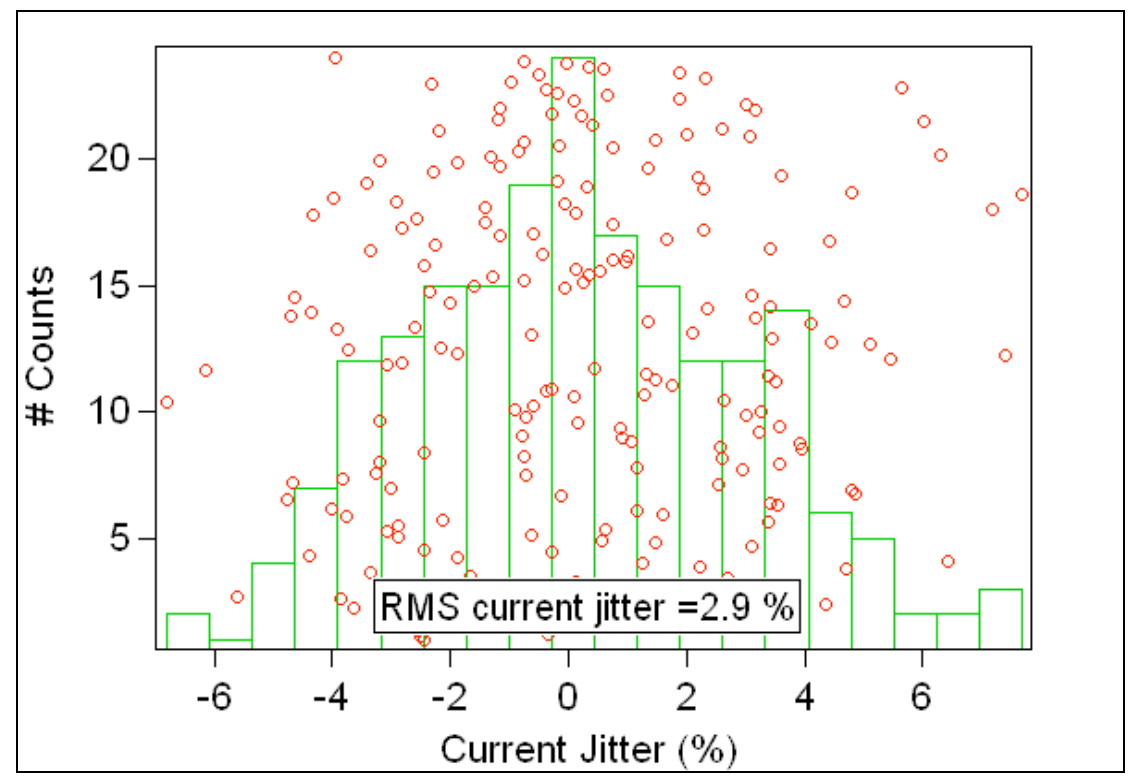

Figure 6.30: Current jitter results for the short bunch case.

In a similar way the energy and the energy spread jitters at the exit of photoinjector have been calculated. Figure 6.31 shows that the RMS energy jitter expected at the end of the 
photoinjector is $\sim 0.24 \%$, that means $\sim 240 \mathrm{keV}$ considering a reference energy of $\sim 100$ $\mathrm{MeV}$.

Concerning the energy spread, the RMS jitter obtained corresponds to few eVs: this means that in this configuration the energy spread can be assumed constant.

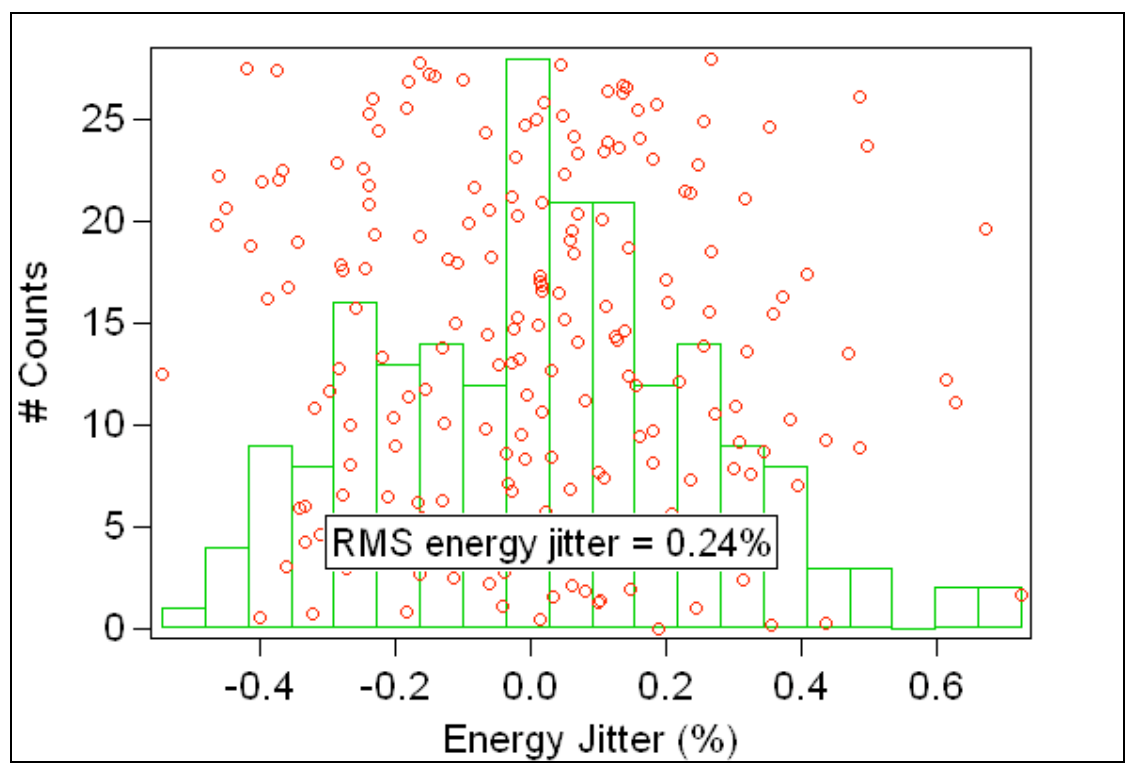

Figure 6.31: Energy jitter results for the short bunch case.

\section{Medium Bunch}

Concerning the medium bunch case, the time, energy, energy spread, current and emittance jitters have been studied for the flat-top case and for the ramped current case.

\section{Flat-top current profile}

Sensitivity studies of each input injector parameters to the time, energy, energy spread, current and emittance jitter have been performed. In particular we have evaluated by using ASTRA and GPT the minimum variation of each parameter providing a certain variation in the outputs. Table 6.2 shows the obtained results.

These results are useful to identify the main sources of jitter. For example concerning the time jitter, the main contributions come from the Gun (accelerating gradient and RF phase) and from the laser time jitter, whose stability requirements will be required to be much tighter.

Similarly to the short bunch case, a random gaussian sampling of all the injector parameters according to the tolerances of Table 6.1 has been performed. The laser time jitter has been varied from 300fs down to 100fs. Figure 6.32 shows the outputs relative to the $300 f$ s choice. 
Table 6.2: Minimum parameters variation providing a fixed variation of the outputs, indicated in brackets in the first row. The range we have considered to calculate the sensitivity is reported in brackets in the first column. $(\mathrm{n} / \mathrm{s}=$ not sensitive; $*=$ extrapolated).

\begin{tabular}{|c|c|c|c|c|c|c|}
\hline Parameters (variation) & $\begin{array}{c}\Delta \mathrm{I} \\
(+1 \%)\end{array}$ & $\begin{array}{c}\Delta T \\
(+100 f s)\end{array}$ & $\begin{array}{c}\sigma_{\mathrm{E}} \\
(10 \mathrm{keV})\end{array}$ & $\begin{array}{c}\Delta E / E \\
(+0.1 \%)\end{array}$ & $\begin{array}{c}\varepsilon_{\text {proj }} \\
(10 \%)\end{array}$ & $\begin{array}{c}<\varepsilon_{\text {slice }}> \\
10 \%\end{array}$ \\
\hline Gun Solenoid (2\%) & $-1.6 \%$ & $+14.6 \%$ * & $n / s$ & $\mathrm{n} / \mathrm{s}$ & $+0.3 \%$ & $+8.5 \%$ * \\
\hline Gun Eacc (5\%) & $0.5 \%$ & $0.14 \%$ & $2 \%$ & $4 \%$ & $1 \%$ & $2.5 \%$ \\
\hline RF phase gun $\left(1^{\circ}\right)$ & $-1.3^{\circ *}$ & $-0.24^{\circ}$ & $0.32^{\circ}$ & $-2^{\circ}$ & $+3.7^{\circ *}$ & $+5.1^{\circ} *$ \\
\hline Charge $(10 \%)$ & $+1.6 \%$ & $+6.2 \%$ & $>30 \%$ * & $>30 \%$ * & $+6.8 \%$ & $+7.6 \%$ \\
\hline Laser pulse length $(10 \%)$ & $-3.9 \%$ & $>30 \%$ * & $>30 \%$ * & $>30 \%$ * & $-15 \%$ * & $-17 \%$ * \\
\hline Laser time jitter (400fs) & $-500 \mathrm{fs}$ * & 92fs & $-145 \mathrm{fs}$ & 950fs * & 1400fs * & $2900 \mathrm{fs}$ * \\
\hline SOA Eacc (5\%) & $+35 \%$ * & $-2 \%$ & $+2.4 \%$ & $0.21 \%$ & $n / s$ & $n / s$ \\
\hline SOA RF phase $\left(0.2^{\circ}\right)$ & $-6^{\circ *}$ & $-9^{\circ} *$ & $-0.16^{\circ}$ & $+1.1^{\circ *}$ & $\mathrm{n} / \mathrm{s}$ & $\mathrm{n} / \mathrm{s}$ \\
\hline SOB Eacc (5\%) & $\mathrm{n} / \mathrm{s}$ & $\mathrm{n} / \mathrm{s}$ & $16 \%$ * & $0.21 \%$ & $n / s$ & $\mathrm{n} / \mathrm{s}$ \\
\hline SOB RF phase $\left(0.2^{\circ}\right)$ & $\mathrm{n} / \mathrm{s}$ & $\mathrm{n} / \mathrm{s}$ & $-0.3^{\circ *}$ & $+22^{\circ} *$ & $\mathrm{n} / \mathrm{s}$ & $\mathrm{n} / \mathrm{s}$ \\
\hline
\end{tabular}




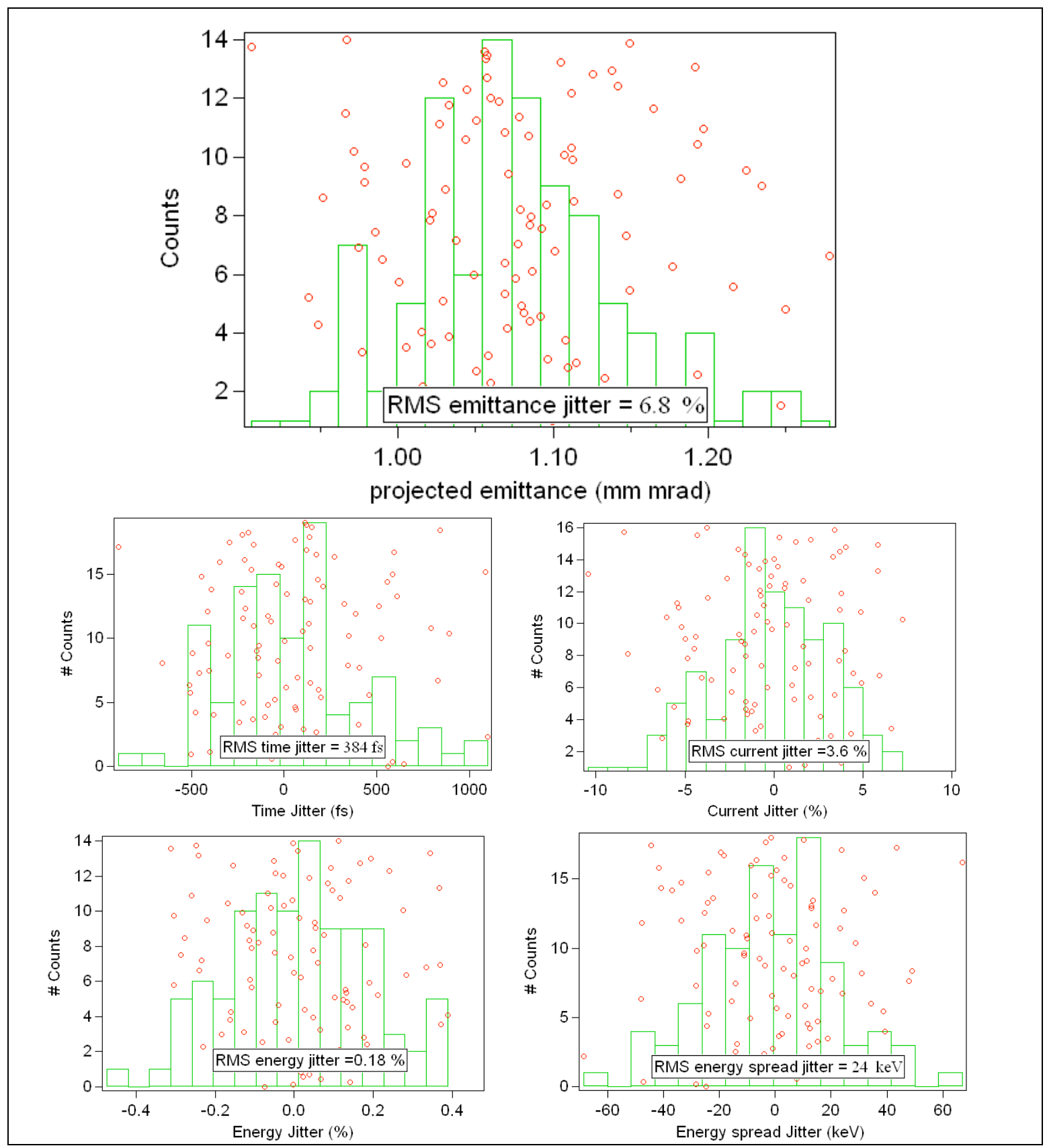

Figure 6.32: Emittance, time, current, energy and energy spread jitters obtained by gaussian sampling the injector parameters according to tolerances in Table 1; laser time jitter=300fs (RMS).

In order to consider the optical matching between the injector and the linac, an analysis of the jitters of the Twiss parameters has been carried out on the same output jittered bunches. Figure 6.33 shows the distributions of $\alpha_{x}$ and $\beta_{x}$ at the exit of the injector. The average $\alpha_{x}$ and $\beta_{x}$ are respectively 0.48 and $18 \mathrm{~m}$, but the jittered outputs are largely spread out with a standard deviation respectively of 0.24 and $3.7 \mathrm{~m}$. This should be taken into account for the finalization of the optics matching. 

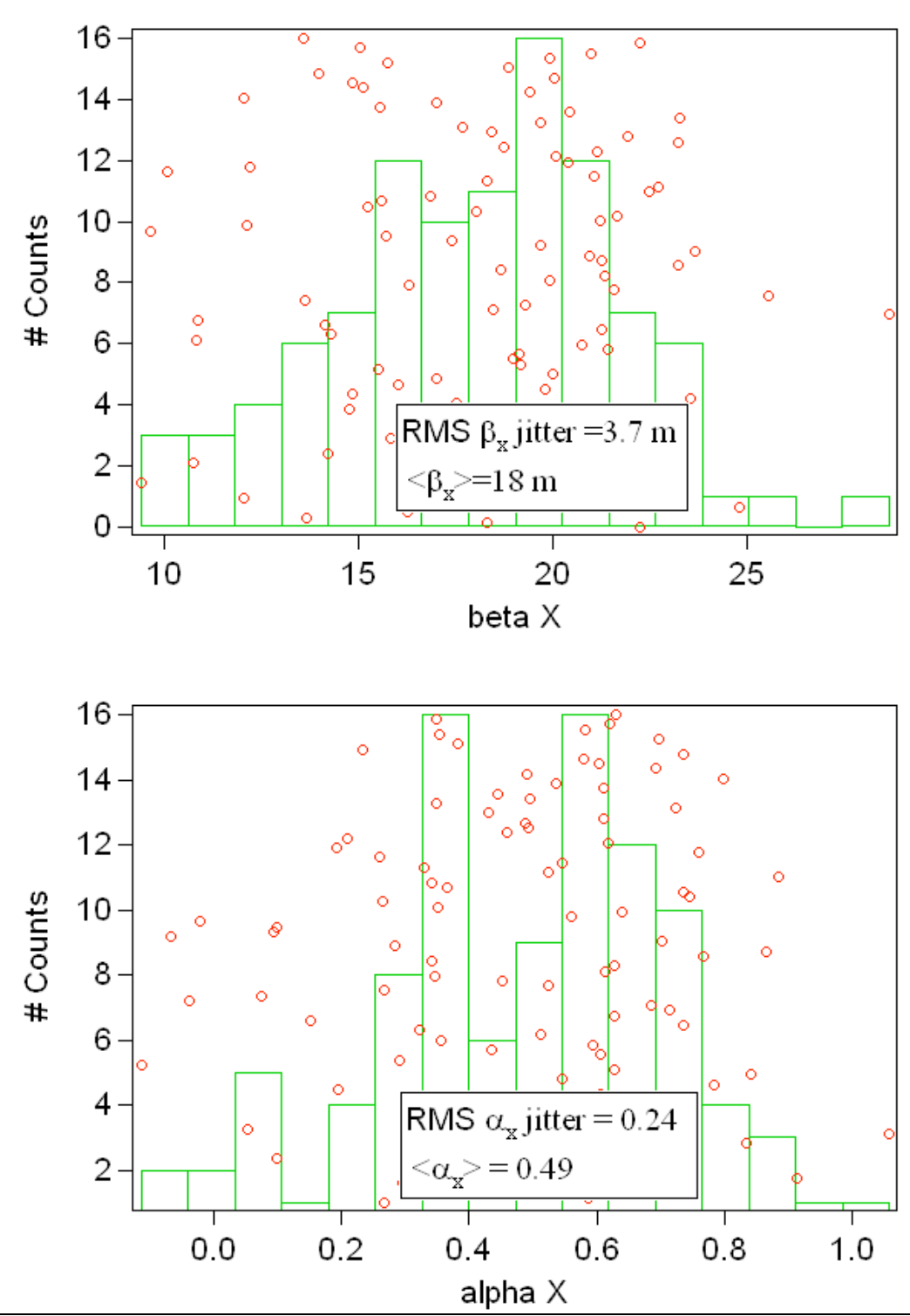

Figure 6.33: $\alpha_{\mathrm{x}}$ and $\beta_{\mathrm{x}}$ jitter at the exit of the photoinjector.

In order to evaluate the potential gain in stability coming from an improvement in the laser time jitter, the jitter study has been repeated assuming laser timing jitters of $200 \mathrm{fs}$ and 100fs. Table 6.4 shows the comparison between the obtained results. The time jitter at the exit of the injector has been improved by reducing the laser time jitter down to $100 \mathrm{fs}$, reaching in this case a RMS value of $250 \mathrm{fs}$. This can be considered the minimum value unless improving the other injector parameters jitters too. 
Table 6.4: Comparison of the output parameter jitter for various laser timing jitters.

\begin{tabular}{|r|c|c|c|}
\hline RMS laser time jitter (fs) & $\mathbf{3 0 0}$ & $\mathbf{2 0 0}$ & $\mathbf{1 0 0}$ \\
\hline Time (fs) & 384 & 332 & 250 \\
\hline Current (\%) & 3.6 & 3.0 & 3.3 \\
\hline Energy (\%) & 0.18 & 0.18 & 0.17 \\
\hline Energy Spread (keV) & 24 & 19 & 17 \\
\hline Emittance (\% $\left.{ }^{\wedge}\right)$ & 6.8 & 6.1 & 6.5 \\
\hline Alpha & 0.49 & 0.27 & 0.24 \\
\hline Beta (m) & 3.7 & 3.0 & 3.7 \\
\hline
\end{tabular}

\section{Ramped current profile}

First of all the sensitivities of only the injector machine parameters have been performed and the results are reported in Table 6.5.

Since the laser pulse shaping process could introduce additional jitters sources, a certain effort has been spent to identify the main pulse shaping jittered parameters, pointing out the correlation between each others. In fact for example a jitter in laser pulse length is transferred to a jitter in the ramping current distribution.

As for the flat-top case a random sampling of all the injector parameters has been performed, taking the RMS values reported in Table 6.1 either for the injector parameters either for the electron bunch features (i.e. charge, time length, spot size and laser time jitters). Two laser time jitter cases have been considered: 200fs and 100fs. Results are reported in Table 6.6. 
Table 6.5: Minimum parameters variation providing a fixed variation of the outputs, indicated in brackets in the first row. The range we have considered to calculate the sensitivity is reported in brackets in the first column. (n/s $=$ not sensitive; * = extrapolated).

\begin{tabular}{|c|c|c|c|c|c|c|}
\hline Parameters (variation) & $\begin{array}{c}\Delta \mathrm{I} \\
(+1 \%)\end{array}$ & $\begin{array}{c}\Delta T \\
(+100 f s)\end{array}$ & $\begin{array}{c}\sigma_{\mathrm{E}} \\
(10 \mathrm{keV})\end{array}$ & $\begin{array}{c}\Delta E / E \\
(+0.1 \%)\end{array}$ & $\begin{array}{c}\varepsilon_{\text {proj }} \\
(10 \%)\end{array}$ & $\begin{array}{c}<\varepsilon_{\text {slice }}> \\
10 \%\end{array}$ \\
\hline Gun Solenoid (2\%) & $-1.5 \%$ & $+10.7 \%$ * & $1.1 \%$ & $\mathrm{n} / \mathrm{s}$ & $+0.2 \%$ & $+2 \%$ \\
\hline Gun Eacc (1\%) & $0.47 \%$ & $0.13 \%$ & $0.12 \%$ & $0.96 \%$ & $0.33 \%$ & $4.3 \%$ \\
\hline RF phase gun $\left(1^{\circ}\right)$ & $0.65^{\circ} *$ & $2.8^{\circ}$ & $0.8^{\circ}$ & $3.8^{\circ}$ & $0.5^{\circ} *$ & $4.8^{\circ} *$ \\
\hline SOA Eacc (5\%) & $20 \%$ * & $2 \%$ & $3.9 \%$ & $0.21 \%$ & $n / s$ & $n / s$ \\
\hline SOA RF phase $\left(0.2^{\circ}\right)$ & $6.2^{\circ}$ * & $n / s$ & $0.1^{\circ}$ & $1.9^{\circ}$ * & $\mathrm{n} / \mathrm{s}$ & $n / s$ \\
\hline
\end{tabular}


Table 6.6: Comparison of the output parameter jitter for various laser timing jitters.

\begin{tabular}{|r|c|c|}
\hline RMS laser time jitter (fs) & $\mathbf{2 0 0}$ & $\mathbf{1 0 0}$ \\
\hline Time (fs) & 351 & 279 \\
\hline Current (\%) & 2.4 & 3.7 \\
\hline Energy (\%) & 0.17 & 0.18 \\
\hline Energy Spread (keV) & 42 & 33 \\
\hline Emittance (\%) & 13.1 & 10.3 \\
\hline Alpha & 0.15 & 0.15 \\
\hline Beta (m) & 2.1 & 2.3 \\
\hline
\end{tabular}

Comparing Table 6.4 (flat-top) with Table 6.6 (ramped), the jitter in the outputs for the flat-top and for the ramped cases are very similar except for the emittance. While for the flat-top case it is not sensitive to the laser time jitter (for all the 3 cases it has an rms jitter of $\sim 6 \%$ ), for the ramped case, it has a rms jitter of $13 \%$ and $10 \%$ assuming, respectively, a laser time jitter of $200 \mathrm{fs}$ and $100 \mathrm{fs}$. The issues described above relative to the emittance compensation for a ramped current distribution is reflected in a lessened stability of the optimized injector parameters setting.

\section{Long Bunch}

A simulation campaign has been performed for the long bunch solution in order to define the sensitivity to the input parameters and the output jitters due to the tolerances in the inputs. For the tolerance study, 50k macro-particles were tracked by the ASTRA code for a thousand different cases, generated with a random distribution of the inputs in the ranges defined in the Table 6.1. Particular attention was spent to evaluate the contribution to the jitter in the laser time arrival at the cathode, so three different sets of simulations were performed with $300 \mathrm{fs}$, 200fs and $100 \mathrm{fs}$ rms laser arrival time jitter.

\section{Flat top current profile}

An evaluation of the sensitivity of the 'long flat top' solution to the single parameters is reported in the Table 6.7. 
Table 6.7: Minimum parameters variation providing a fixed variation of the outputs, indicated in brackets in the first row. The range we have considered to calculate the sensitivity is reported in brackets in the first column. $(\mathrm{n} / \mathrm{s}=$ not sensitive; $*=$ extrapolated).

\begin{tabular}{|c|c|c|c|c|c|c|}
\hline Parameters (variation) & $\begin{array}{c}\Delta \mathrm{I} \\
(+1 \%)\end{array}$ & $\begin{array}{c}\Delta T \\
(+100 \mathrm{fs})\end{array}$ & $\begin{array}{c}\sigma_{\mathrm{E}} \\
(10 \mathrm{keV})\end{array}$ & $\begin{array}{c}\Delta E / E \\
(+0.1 \%)\end{array}$ & $\begin{array}{c}\varepsilon_{\text {proj }} \\
(5 \%)\end{array}$ & $\begin{array}{c}\left\langle\varepsilon_{\text {slice }}\right\rangle_{80 \%} \\
(5 \%)\end{array}$ \\
\hline Gun Solenoid (3\%) & $2.3 \%$ & $5 \%$ & $\mathrm{n} / \mathrm{s}$ & $n / s$ & $0.7 \%$ & $0.3 \%$ \\
\hline Gun $E_{a c c}(1 \%)$ & $0.6 \%$ & $0.15 \%$ & $0.2 \%$ & $2.7 \%$ & $0.4 \%$ & $0.7 \%$ \\
\hline Gun RF phase $\left(0.3^{\circ}\right)$ & $1.4^{\circ *}$ & $1^{\circ}$ & $0.3^{\circ}$ & $1.8^{\circ}$ & $3^{\circ *}$ & $2.7^{\circ}$ \\
\hline Charge $(12 \%)$ & $1.5 \%$ & $20 \%$ * & $40 \%$ * & $\mathrm{n} / \mathrm{s}$ & $6 \%$ & $7 \%$ \\
\hline Laser pulse length $(10 \%)$ & $4 \%$ & $2.5 \%$ & $5 \%$ & $n / s$ & $6 \%$ & $9 \%$ \\
\hline Laser time jitter (900fs) & $1 \mathrm{ps}$ & $150 \mathrm{fs}$ & $230 \mathrm{fs}$ & $1.5 p s$ * & $2 p s$ * & $2.5 p s^{*}$ \\
\hline $\operatorname{SOA} E_{a c c}(7 \%)$ & $40 \%$ * & $1.8 \%$ & $1.4 \%$ & $0.25 \%$ & $14 \%$ * & $\mathrm{n} / \mathrm{s}$ \\
\hline SOA RF phase $\left(0.3^{\circ}\right)$ & $6 \%$ * & $0.12^{\circ}$ & $0.27^{\circ}$ & $0.8^{\circ *}$ & $\mathrm{n} / \mathrm{s}$ & $\mathrm{n} / \mathrm{s}$ \\
\hline
\end{tabular}


From the large amount of simulation the statistical analysis was performed to extract the RMS fluctuation of the several output beam properties. In the following Figures are shown the distribution histograms of several output bunch parameters.

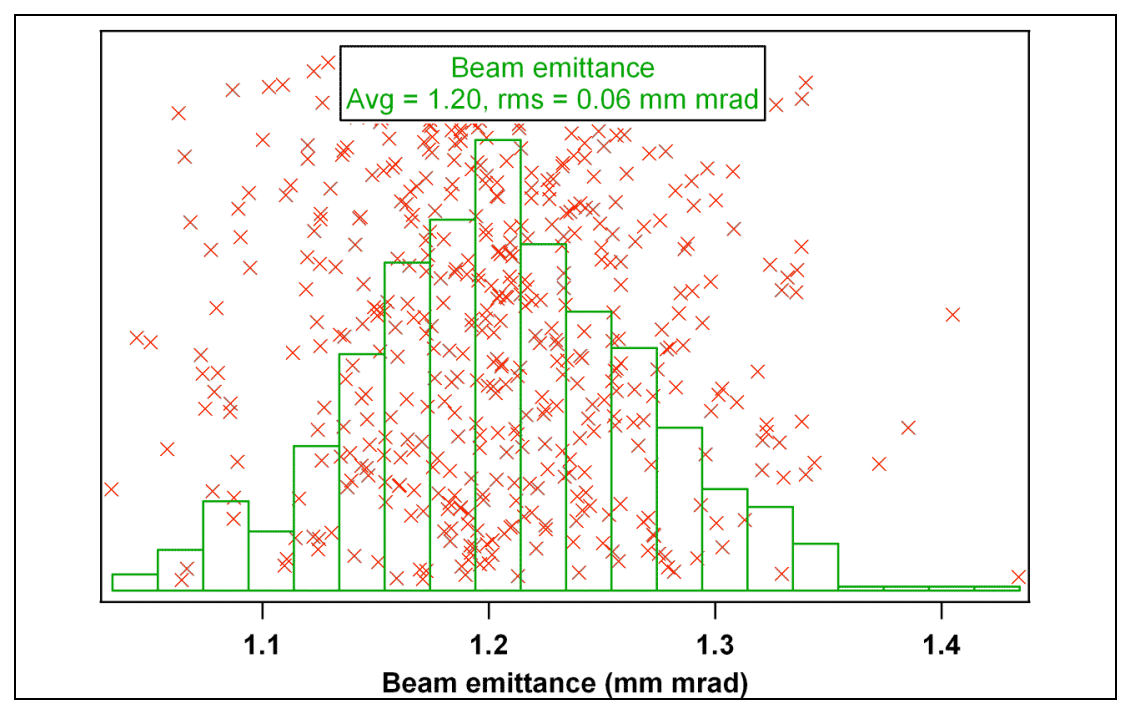

Figure 6.34: Emittance jitter distribution at the exit of the photoinjector.

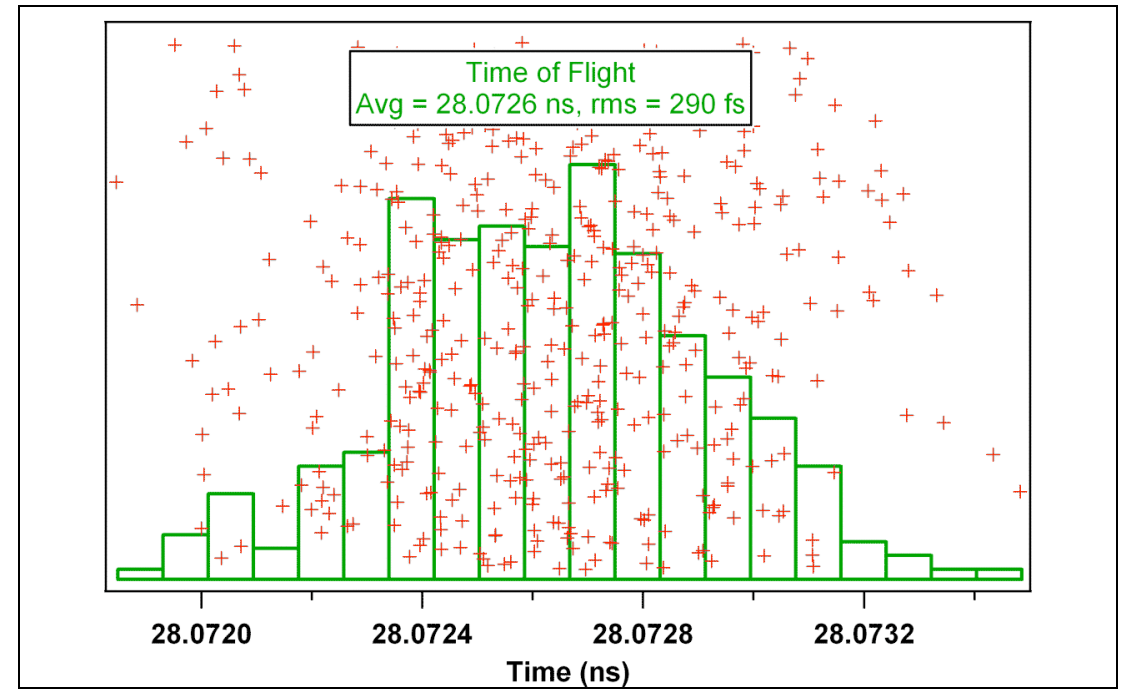

Figure 6.35: Time of flight jitter of the bunch in the injector system. 

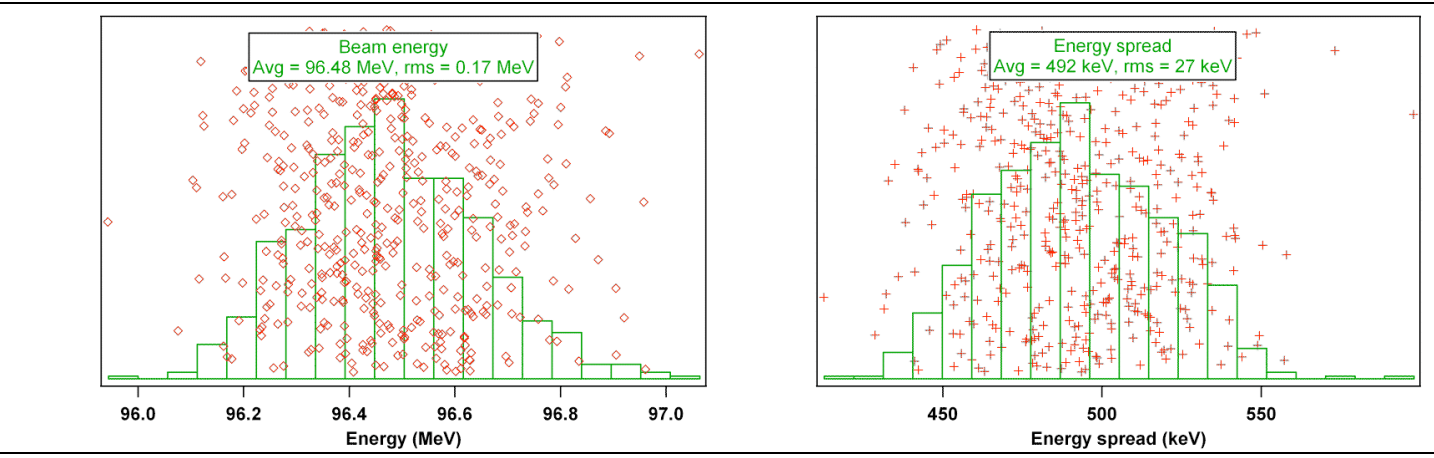

Figure 6.36: Beam energy and energy spread jitter distribution.
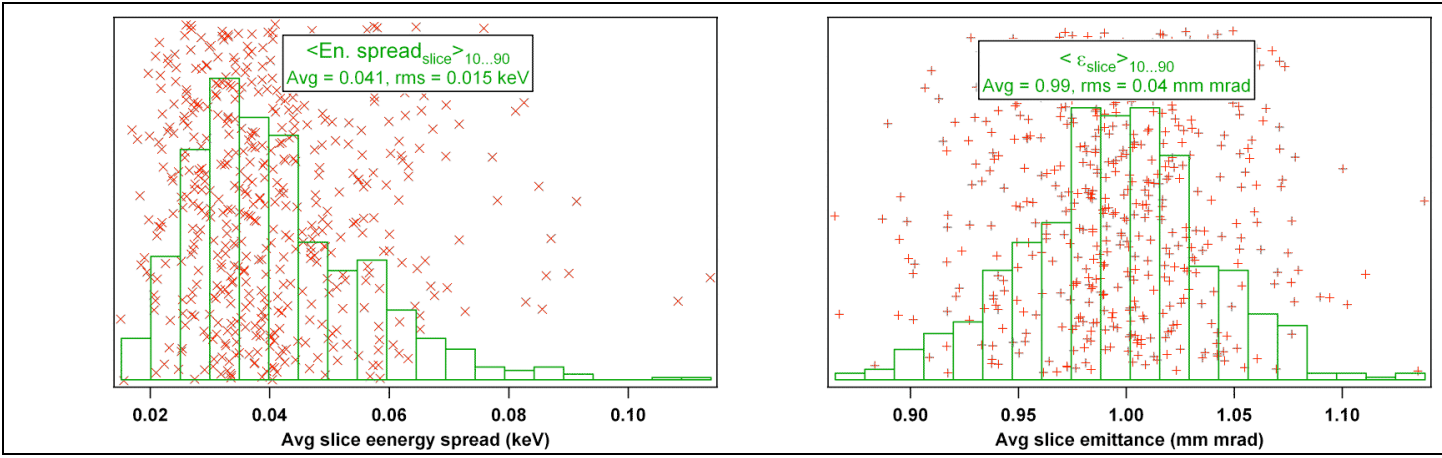

Figure 6.37: Slice energy spread and slice emittance jitter distribution.
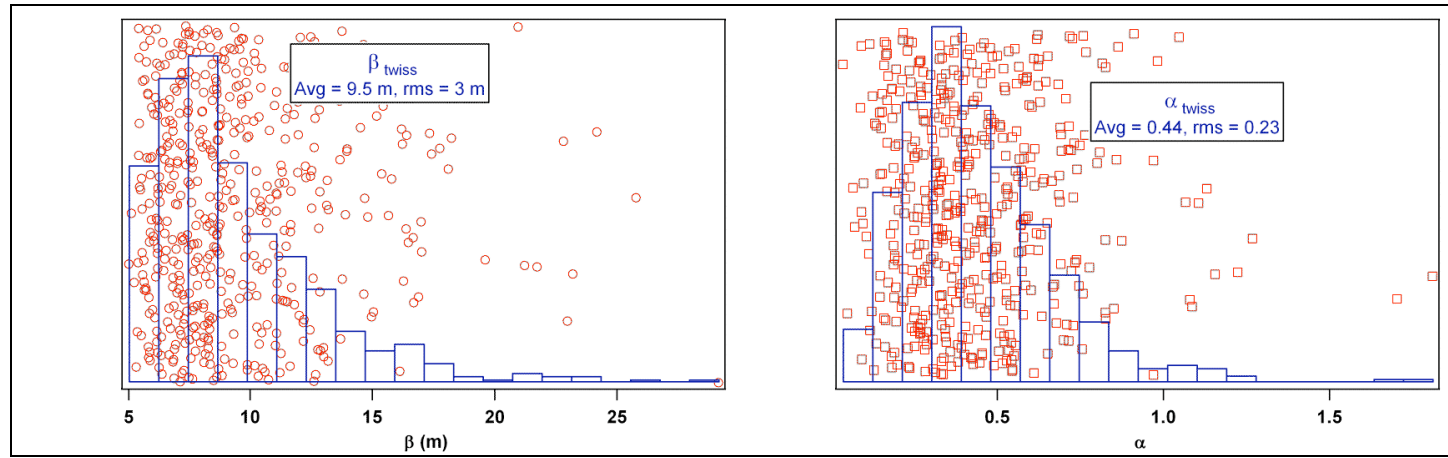

Figure 6.38: $\alpha_{x}$ and $\beta_{x}$ jitter at the exit of the photoinjector.

Table 6.8 summarizes all jitters presented in the previous plots and presents the results for the three simulation sets with different laser arrival time jitter. 
Table 6.8: Comparison of the output parameter jitter for various laser timing jitters.

\begin{tabular}{|r|c|c|c|}
\hline RMS laser time jitter (fs) & $\mathbf{3 0 0}$ & $\mathbf{2 0 0}$ & $\mathbf{1 0 0}$ \\
\hline Time (fs) & $\mathbf{2 9 0}$ & 250 & $\mathbf{2 2 4}$ \\
\hline Current (\%) & 3.1 & 3.1 & 3.1 \\
\hline Energy (\%) & 0.17 & 0.17 & 0.17 \\
\hline Uncorrelated Energy Spread (eV) & 17 & 21 & 18 \\
\hline Emittance (\%) & 5.4 & 5.1 & 17 \\
\hline Slice Emittance (\%) & 4.2 & 3.9 & 4.1 \\
\hline Alpha & 0.23 & 0.27 & 0.24 \\
\hline Beta (m) & 3 & 3.3 & 2.7 \\
\hline
\end{tabular}

\section{Ramped current profile}

Sensitivities of only the injector machine parameters have been so far performed also for the long ramped bunch case and the results are reported in Table 6.9.

As for the flat-top case a random sampling of the injector parameters (following Table $6.1)$ has been performed. Three laser time jitters case have been considered: $300 \mathrm{fs}, 200 \mathrm{fs}$ and 100fs. Results are reported in Table 6.10. 
Table 6.9: Minimum parameters variation providing a fixed variation of the outputs, indicated in brackets in the first row. The range we have considered to calculate the sensitivity is reported in brackets in the first column. (n/s $=$ not sensitive; * = extrapolated).

\begin{tabular}{|c|c|c|c|c|c|c|}
\hline Parameters (variation) & $\begin{array}{c}\Delta I \\
(+1 \%)\end{array}$ & $\begin{array}{c}\Delta T \\
(+100 \text { fs })\end{array}$ & $\begin{array}{c}\sigma_{\mathrm{E}} \\
(10 \mathrm{keV})\end{array}$ & $\begin{array}{c}\Delta E / E \\
(+0.1 \%)\end{array}$ & $\begin{array}{c}\varepsilon_{\text {proj }} \\
(5 \%)\end{array}$ & $\begin{array}{c}\left\langle\varepsilon_{\text {slice }}>_{80 \%}\right. \\
(5 \%)\end{array}$ \\
\hline Gun Solenoid (3\%) & $2.2 \%$ & $10 \% *$ & $6 \%$ * & $\mathrm{n} / \mathrm{s}$ & $0.8 \%$ & $1.2 \%$ \\
\hline Gun $E_{\mathrm{acc}}(1 \%)$ & $0.6 \%$ & $0.15 \%$ & $0.17 \%$ & $1.6 \%$ & $0.5 \%$ & $0.9 \%$ \\
\hline Gun RF phase $\left(0.4^{\circ}\right)$ & $1^{\circ *}$ & $0.3^{\circ}$ & $0.28^{\circ}$ & $1.8^{\circ}$ & $2.9^{\circ *}$ & $2.3^{\circ *}$ \\
\hline SOA E $E_{\text {acc }}(7 \%)$ & $\mathrm{n} / \mathrm{s}$ & $2 \%$ & $1.1 \%$ & $0.2 \%$ & $15 \%$ * & $\mathrm{n} / \mathrm{s}$ \\
\hline SOA RF phase $\left(0.3^{\circ}\right)$ & $\mathrm{n} / \mathrm{s}$ & $\mathrm{n} / \mathrm{s}$ & $0.22^{\circ}$ & $0.7^{\circ *}$ & $\mathrm{n} / \mathrm{s}$ & $\mathrm{n} / \mathrm{s}$ \\
\hline
\end{tabular}


Table 6.10: Comparison between the outputs jitters assuming a laser time jitter of $300 \mathrm{fs}$, $200 \mathrm{fs}$ and $100 \mathrm{fs}$.

\begin{tabular}{|r|c|c|c|}
\hline RMS laser time jitter (fs) & $\mathbf{3 0 0}$ & $\mathbf{2 0 0}$ & $\mathbf{1 0 0}$ \\
\hline Time (fs) & 289 & 236 & 220 \\
\hline Current (\%) & 3.2 & 3.2 & 3.2 \\
\hline Energy (\%) & 0.17 & 0.17 & 0.17 \\
\hline Energy Spread (keV) & 27 & 23 & 22 \\
\hline Uncorrelated En. Spr. (eV) & 6 & 6 & 6 \\
\hline Emittance (\%) & 6.1 & 6.1 & 6 \\
\hline Slice Emittance (\%) & 4 & 4 & 4 \\
\hline Alpha & 0.18 & 0.18 & 0.18 \\
\hline Beta (m) & 4.8 & 4.8 & 4.8 \\
\hline
\end{tabular}

\section{Further Output analysis}

A further analysis of the injector output bunches from the jitter simulations has been implemented. Polynomial fittings of the longitudinal phase space, current bunch profile and slice emittance have been performed for each simulated case.

Figure 6.34 shows twelve fourth order polynomial curve fits of the longitudinal phase spaces of the bunches simulated for the long ramped case (100fs laser arrival time jitter sub-sets). Figure 6.35 shows the current profile cases and Figure 6.36 shows the emittance slice behavior along the bunch. The curves show the synchronous bunch core $(3 \mathrm{~mm})$ while the tails are missed.

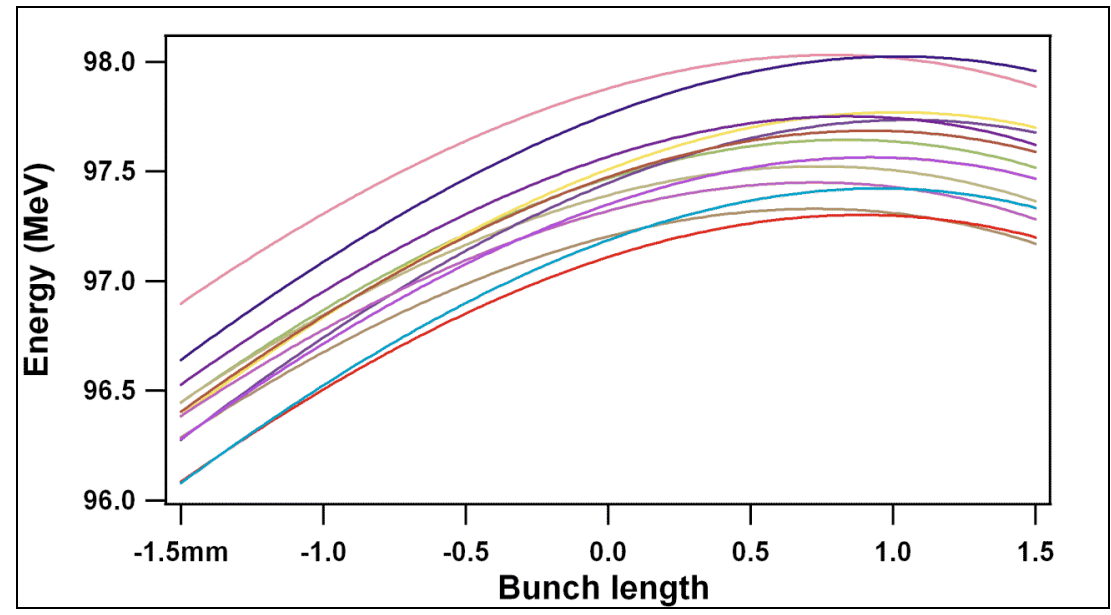

Figure 6.34: 12 polynomial curve fits (fourth order), randomly sampled in the thousands performed, of the longitudinal phase spaces of bunches simulated for the long ramped case. Bunch head is on the left. 


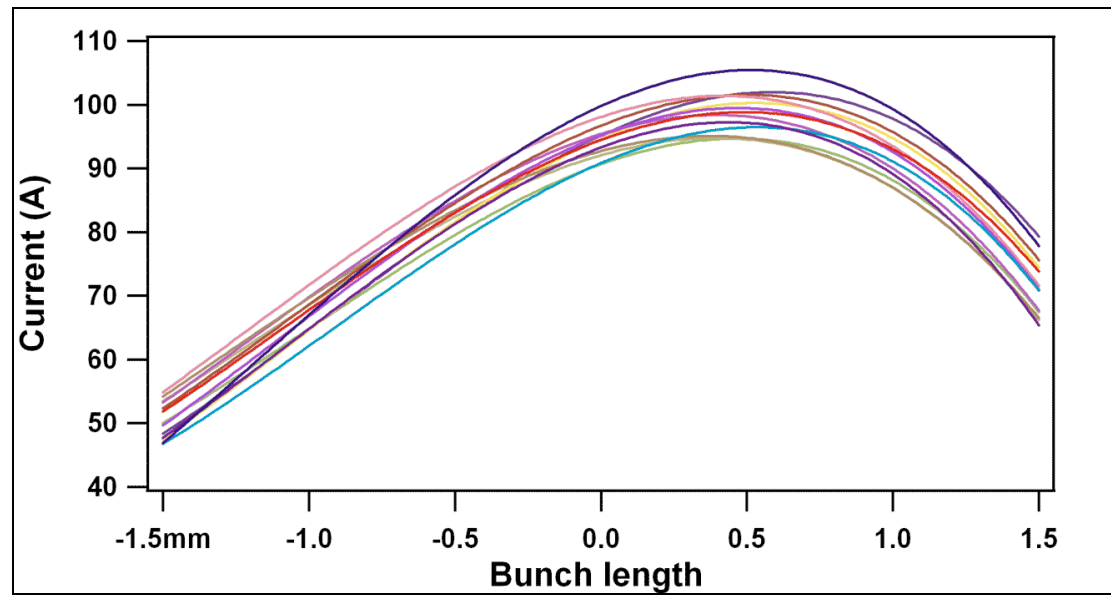

Figure 6.35: 12 polynomial curve fits (fourth order), randomly sampled in the thousands performed, of the current profile of bunches simulated for the long ramped case. Bunch head is on the left.

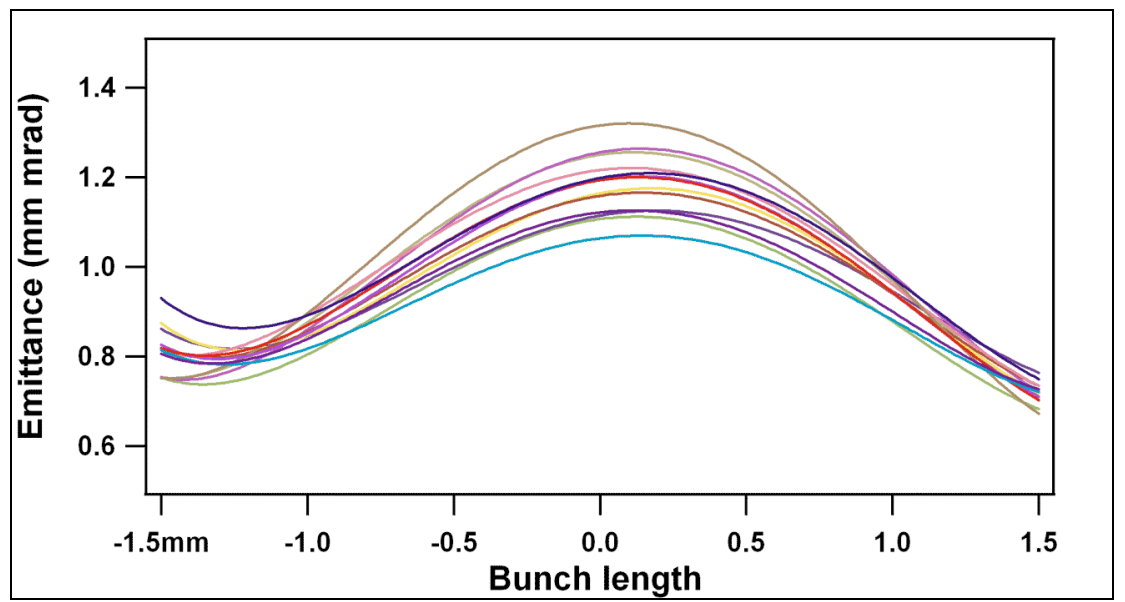

Figure 6.36: 12 polynomial curve fits (fifth order), randomly sampled in the thousands performed, of the slice emittance profile of bunches simulated for the long ramped case.

Bunch head is on the left.

Each fit provides the polynomial coefficients and, after fitting all simulations, a statistical analysis of the coefficients can be performed. Thus, if the $f_{i}(z)=c 0_{i}+c 1_{i} \cdot z+c 2_{i} \cdot z^{2}+\ldots$ is the function describing different curves, where $i$ is the simulation index, an average can be done over the $i$ range. Table 6.11 reports the analysis result for the three cases studied (phase space, current and slice). These coefficients can be used to reconstruct analytically the injector output particle distribution with respect to the jitter, allowing a generation in only a bi-dimensional space. This can be useful when there is unique interest into longitudinal phase space evaluation, like in the case of the LiTrack code use. 
Table 6.11: Statistical results performed over the fitting coefficients. The curve function used is a polynomial as: $f_{i}(z)=c 0_{i}+c 1_{i} \cdot z+c 2_{i} \cdot z^{2}+\ldots$.

\begin{tabular}{|c|c|c|c|}
\hline Fit coefficient & $\begin{array}{c}\text { Longitudinal } \\
\text { phase space }\end{array}$ & $\begin{array}{c}\text { Current } \\
\text { profile }\end{array}$ & $\begin{array}{c}\text { Slice } \\
\text { emittance }\end{array}$ \\
\hline$<c 0>$ & 97.38 & 93 & 1.17 \\
\hline $\mathrm{c} 0_{\mathrm{sdv}}$ & 0.17 & 3 & 0.06 \\
\hline$<\mathrm{c} 1>$ & 414 & $1.710^{4}$ & 97 \\
\hline $\mathrm{c} 1_{\mathrm{sdv}}$ & 43 & $210^{3}$ & 22 \\
\hline$<\mathrm{c} 2>$ & $-2.1810^{5}$ & $-1.4410^{7}$ & $3.610^{5}$ \\
\hline $\mathrm{c} 2_{\mathrm{sdv}}$ & $410^{3}$ & $1.210^{6}$ & $410^{4}$ \\
\hline$<\mathrm{c} 3>$ & $-2.1110^{7}$ & $-4.4510^{9}$ & $-5.810^{8}$ \\
\hline $\mathrm{c} 3_{\mathrm{sdv}}$ & $1.910^{6}$ & $3.710^{8}$ & $1.110^{7}$ \\
\hline$<\mathrm{c} 4>$ & - & - & $7.710^{10}$ \\
\hline $\mathrm{c} 4 \mathrm{sdv}$ & - & - & $810^{9}$ \\
\hline
\end{tabular}

\subsection{References}

[6.1] S. Di Mitri, et. al. ,'Optimization and Modeling of the Accelerator for the FERMI @ Elettra FEL", Proceedings of the $27^{\text {th }}$ International Free Electron Laser Conference, Stanford, 2005.

[6.2] G. De Ninno, private communication.

[6.3] S. Di Mitri, ST/F-TN-05/17 (2005).

[6.4] W.S Graves et al., "Measurement of thermal emittance for a Copper Photocathode", PAC 2001.

[6.5] L. Serafini and J. Rosenzweig, "Envelope analysis of intense relativistic quasilaminar beams in RF photoinjectors: a theory of emittance compensation", Phys. Rev. E 55, 7565 (1997).

[6.6] A. Zholents, et. al., Linac optimization Technical Note, ST/F-TN-06/??

[6.7] G. Penco, "Longitudinal Space Charge Field at the Cathode In Case of a Non Uniform Bunch", ST/F-TN-06/03 (2006).

[6.8] SLAC Report No. SLAC-R-593 (2002).

[6.9] G. Penco, M. Trovo', “Time Jitter from the Photoinjector”, ST/F-TN-05/25 (2005). 


\section{Conclusions}

We have presented an injector design based on proven technologies and techniques, but that is nonetheless suitable for producing the high-brightness electron beams required for successful operation of the FERMI @ Elettra FELs.

Photocathode performance specifications are well established and conservative. Laser systems are aggressive in average and peak power, but benefit from parallel development tracks at other laboratories and manufacturers. The laser profile tailoring is critical, and will require development effort to produce suitably reliable pulses at the photocathode.

The design of the off-axis diagnostic beamline is presented in a somewhat rudimentary stage and will benefit from additional development studies. However, it remains flexible enough to determine the beam energy and energy spread. Additional measurement modes include longitudinal phase space correlations and thermal emittance in low charge per bunch beams.

We have presented an analysis of the transient behavior of the pi and zero modes of the RF gun under the assumption of a particular RF drive source complex waveform. We have analyzed the sensitivity of the baseline beam dynamic solution for the medium bunch case to the influence of the zero mode excitation for various RF gun cavity geometries corresponding to varying frequency separation between the pi and zero cavity modes. For the length and intensity of the electron beam bunches under consideration, we have found a small effect on the transverse beam quality, but a more profound effect on the longitudinal phase space. We have shown heightened sensitivity to the energy correlations present in the at the RF gun exit to the larger amplitude perturbations resulting from smaller mode frequency separation.

We have optimized the beamline parameters and incident drive laser profile have demonstrated a flexible beamline design that can accommodate various operating modes of the linac to supply high brightness electron beams that are suitable for generating a variety of seeded-FEL x-ray pulses.

We have demonstrated the production and transport of a charge bunch with large initial nonlinear variation of the instantaneous current. These beam have been shown to undergo redistribution of charge such that a linear current ramp is developed over much of the bunch at the exit of the injector. These beams have been shown to be amenable to standard emittance compensation techniques.

We have analyzed the parameter sensitivity of the baseline. The most significant element of the jitter budget remains the timing jitter at the exit of the injector, with the majority of the contribution arising from the drive laser arrival time jitter. For the medium bunch cases, output timing jitter of $\sim 350$ fs or less is achievable for drive laser arrival time jitter of $200 \mathrm{fs}$ or less, while the long bunch cases have somewhat more relaxed tolerances. 